\title{
ON HIGHER DIRECT IMAGES OF CONVERGENT ISOCRYSTALS
}

\author{
DAXIN XU
}

\begin{abstract}
Let $k$ be a perfect field of characteristic $p>0$ and $\mathrm{W}$ the ring of Witt vectors of $k$. In this article, we give a new proof of the Frobenius descent for convergent isocrystals on a variety over $k$ relative to $\mathrm{W}$. This proof allows us to deduce an analogue of the de Rham complexes comparaison theorem of Berthelot [8] without assuming a lifting of the Frobenius morphism. As an application, we prove a version of Berthelot's conjecture on the preservation of convergent isocrystals under the higher direct image by a smooth proper morphism of $k$-varieties.
\end{abstract}

\section{CONTEnts}

1. Introduction

2. Preliminary

3. Crystalline cohomology for isocrystals

4. Convergent topos and convergent isocrystals

5. Higher direct images of a convergent isocrystal are $p$-adically convergent

6. Frobenius descents

7. Review on rigid geometry

8. Rigid convergent topos and convergent isocrystals

9. Higher direct images of a convergent isocrystal References

\section{INTRODUCTION}

1.1. Let $k$ be a perfect field of characteristic $p>0$. A good $p$-adic cohomology theory on a variety over $k$ is the rigid cohomology developed by Berthelot [5, 6]. The coefficients for this theory are (over-)convergent $F$-isocrystals: they play a similar role of the lisse $\ell$-adic sheaves in $\ell$-adic cohomology. In ([5] 4.3, [33]), Berthelot and Tsuzuki conjectured that under a smooth proper morphism of varieties over $k$, the higher direct image of a (over-)convergent $(F$-)isocrystal is still a (over-)convergent $(F$-)isocrystal analogue to the $\ell$-adic case. Various cases and variants of this conjecture have been proved by Tsuzuki [33], Shiho [32], Étess [17, Caro [14, etc. We refer to an article of Lazda 24] for a survey on these results and the relation between them. The goal of this article is to prove a version of Berthelot's conjecture in the context of convergent topos developed by Ogus (1.9).

1.2. In [26, 27], Ogus introduced a crystalline-like site: convergent site and defined a convergent isocrystal as a crystal on this site. Let us briefly recall his definition.

Date: February 27, 2018. 
Let $\mathrm{W}$ be the ring of Witt vectors of $k, K$ its fraction field and $X$ a scheme of finite type over $k$. We denote by $\operatorname{Conv}(X / \mathrm{W})$ the category of couples $(\mathfrak{T}, u)$ consisting of a flat formal W-scheme of finite type $\mathfrak{T}$ and a $k$-morphism $u$ from the reduced subscheme $T_{0}$ of the special fiber of $\mathfrak{T}$ to $X$. Morphisms are defined in a natural way. A family of morphisms $\left\{\left(\mathfrak{T}_{i}, u_{i}\right) \rightarrow(\mathfrak{T}, u)\right\}_{i \in I}$ is a covering if $\left\{\mathfrak{T}_{i} \rightarrow \mathfrak{T}\right\}_{i \in I}$ is a Zariski covering.

The functor $(\mathfrak{T}, u) \mapsto \Gamma\left(\mathfrak{T}_{\text {zar }}, \mathscr{O}_{\mathfrak{T}}\left[\frac{1}{p}\right]\right)$ is a sheaf of rings that we denote by $\mathscr{O}_{X / K}$. An $\mathscr{O}_{X / K}$-module amounts to give the following data:

(i) for every object $(\mathfrak{T}, u)$ of $\operatorname{Conv}(X / \mathrm{W})$, an $\mathscr{O}_{\mathfrak{T}}\left[\frac{1}{p}\right]$-module $\mathscr{F}_{\mathfrak{T}}$ of $\mathfrak{T}_{\text {zar }}$,

(ii) for every morphism $f:\left(\mathfrak{T}_{1}, u_{1}\right) \rightarrow\left(\mathfrak{T}_{2}, u_{2}\right)$ of $\operatorname{Conv}(X / \mathrm{W})$, an $\mathscr{O}_{\mathfrak{T}_{1}}$-linear morphism $c_{f}$ : $f^{*}\left(\mathscr{F}_{\mathfrak{T}_{2}}\right) \rightarrow \mathscr{F}_{\mathfrak{T}_{1}}$,

satisfying a cocycle condition for the composition of morphisms as in ([11] 5.1).

A convergent isocrystal on $\operatorname{Conv}(X / \mathrm{W})$ is a coherent crystal of $\mathscr{O}_{X / K^{-}}$-modules $\mathscr{F}$ on $\operatorname{Conv}(X / \mathrm{W})$, i.e. for every object $(\mathfrak{T}, u)$ of $\operatorname{Conv}(X / \mathrm{W}), \mathscr{F}_{\mathfrak{T}}$ is coherent, and for every morphism $f$ of $\operatorname{Conv}(X / \mathrm{W})$, the transition morphism $c_{f}$ is an isomorphism. We denote by $\operatorname{Iso}^{\dagger}(X / \mathrm{W})$ the category of convergent isocrystals on $\operatorname{Conv}(X / \mathrm{W})$. If $X$ is smooth over $k$, a convergent isocrystal can be viewed as the isogeny class of a crystal of $\mathscr{O}_{X / \mathrm{W}}$-module on the crystalline site $\operatorname{Crys}(X / \mathrm{W})$ satisfying certain convergent conditions (cf. 27] 0.7.2 and [6] 2.2.14).

1.3. In ([26] 4.6), Ogus showed that the category $\operatorname{Iso}^{\dagger}(X / \mathrm{W})$ satisfies descent property under a proper and surjective morphism of $k$-schemes. Then, if $X^{\prime}$ denotes the base change of $X$ by the Frobenius morphism of $k$, the functorial morphism of convergent topoi induced by the relative Frobenius morphism $F_{X / k}: X \rightarrow X^{\prime}$ gives an equivalence of categories:

$$
F_{X / k, \text { conv }}^{*}: \operatorname{Iso}^{\dagger}\left(X^{\prime} / \mathrm{W}\right) \stackrel{\sim}{\rightarrow} \operatorname{Iso}^{\dagger}(X / \mathrm{W}),
$$

that we call Frobenius descent.

A convergent $F$-isocrystal on $\operatorname{Conv}(X / \mathrm{W})$ is a couple $(\mathscr{E}, \varphi)$ of a convergent isocrystal $\mathscr{E}$ on $\operatorname{Conv}(X / \mathrm{W})$ and an isomorphism $\varphi$ between $\mathscr{E}$ and its pullback via the absolute Frobenius morphism of $X$ (cf. 6.14 for a precise definition).

1.4. To study the higher direct image of convergent $(F-)$ isocrystals, we need the notion of convergent topos over a $p$-adic base developed by Shiho [30, 31] 1]. Let $\mathfrak{S}$ be a flat formal W-scheme of finite type, $S_{0}$ the reduced subscheme of its special fiber and $X$ an $S_{0}$-scheme. We define the convergent site $\operatorname{Conv}(X / \mathfrak{S})$ of $X$ relative to $\mathfrak{S}$ and the category $\operatorname{Iso}^{\dagger}(X / \mathfrak{S})$ of convergent isocrystals on $\operatorname{Conv}(X / \mathfrak{S})$ as in 1.2 (cf. 4.1 and 4.14). Shiho generalized Ogus' proper surjective descent for convergent isocrystals in this setting ([32] 7.3).

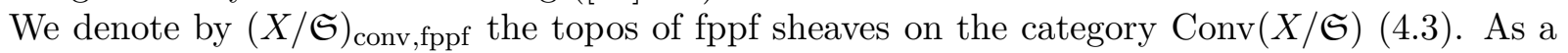
first step towards Berthelot's conjecture, we show the following result.

Theorem 1.5 (6.6). Suppose that the Frobenius morphism $F_{S_{0}}: S_{0} \rightarrow S_{0}$ is flat. Let $X$ be an $S_{0}$-scheme locally of finite type, $X^{\prime}=X \times_{S_{0}, F_{S_{0}}} S_{0}$ and $F_{X / S_{0}}: X \rightarrow X^{\prime}$ the relative Frobenius morphism. The functorial morphism of topoi $F_{X / S_{0}, \mathrm{conv}}:(X / \mathfrak{S})_{\mathrm{conv}, \mathrm{fppf}} \rightarrow\left(X^{\prime} / \mathfrak{S}\right)_{\mathrm{conv}, \mathrm{fppf}}$ is an equivalence of topoi.

\footnotetext{
${ }^{1}$ Actually, Shiho developed a theory of log convergent site and log convergent cohomology over a $p$-adic base with $\log$ structure.
} 
Our proof is inspired by a site-theoretic construction of the Cartier transform of Ogus-Vologodsky due to Oyama [28, 29] and its lifting modulo $p^{n}$ developed by the author [34]. By Gabber-BoschGörtz's faithfully flat descent theory for coherent sheaves in rigid geometry [12], we obtain a new proof of the Frobenius descent (1.3.1).

Corollary 1.6 (6.7). Keep the hypotheses of 1.5. The direct image and inverse image functors of $F_{X / S_{0}, \text { conv }}$ induce equivalences of categories quasi-inverse to each other:

$$
\operatorname{Iso}^{\dagger}(X / \mathfrak{S}) \leftrightarrows \operatorname{Iso}^{\dagger}\left(X^{\prime} / \mathfrak{S}\right)
$$

1.7. Keep the notation of [1.5] and suppose that there exists smooth liftings $\mathfrak{X}$ of $X$ and $\mathfrak{X}^{\prime}$ of $X^{\prime}$ over $\mathfrak{S}$ (in particular, $X$ is smooth over $S_{0}$ ). We denote by $\widehat{\Omega}_{\mathfrak{X} / \mathfrak{S}}^{1}$ the $\mathscr{O}_{\mathfrak{X} \text {-module of differentials }}$ of $\mathfrak{X}$ relative to $\mathfrak{S}$. Given a convergent isocrystal $\mathscr{E} \in \mathbf{O b}\left(\operatorname{Iso}^{\dagger}(X / \mathfrak{S})\right)$, there exists an integrable connection $\nabla: \mathscr{E}_{\mathfrak{X}} \rightarrow \mathscr{E}_{\mathfrak{X}} \otimes_{\mathscr{O}_{\mathfrak{X}}} \widehat{\Omega}_{\mathfrak{X} / \mathfrak{S}}^{1}$ on the coherent $\mathscr{O}_{\mathfrak{X}}\left[\frac{1}{p}\right]$-module $\mathscr{E}_{\mathfrak{X}}$ (4.15). We denote by $\mathscr{E}_{\mathfrak{X}} \otimes_{\mathscr{O}_{\mathfrak{X}}} \widehat{\Omega}_{\mathfrak{X} / \mathfrak{S}}^{\bullet}$ the associated de Rham complex. We deduce from 1.5 the following result about comparing de Rham complexes for the Frobenius descent:

Corollary 1.8 (6.9). Keep the above notation and let $f: X \rightarrow S_{0}$ be the canonical morphism. There exists a canonical isomorphism between de Rham complexes of $\mathscr{E}$ and of $F_{X / S_{0}, \operatorname{conv} *}(\mathscr{E})$ in $\mathrm{D}\left(X_{\mathrm{zar}}^{\prime}, f^{-1}\left(\mathscr{O}_{\mathfrak{S}}\right)\right)$ :

$$
F_{X / S_{0} *}\left(\mathscr{E}_{\mathfrak{X}} \otimes_{\mathscr{O}_{\mathfrak{X}}} \widehat{\Omega}_{\mathfrak{X} / \mathfrak{S}}^{\bullet} \stackrel{\sim}{\rightarrow}\left(F_{X / S_{0}, \operatorname{conv} *}(\mathscr{E})\right)_{\mathfrak{X}^{\prime}} \otimes_{\mathscr{O}_{\mathfrak{X}^{\prime}}} \widehat{\Omega}_{\mathfrak{X}^{\prime} / \mathfrak{S}}^{\bullet}\right.
$$

In [7], Berthelot introduced a sheaf $\mathscr{D}^{\dagger}$ of differential operators over $\mathfrak{X}$ and described (over)convergent isocrystals in terms of arithmetic $\mathscr{D}^{\dagger}$-modules. If there exists a lifting $F: \mathfrak{X} \rightarrow \mathfrak{X}^{\prime}$ of the relative Frobenius morphism $F_{X / S_{0}}$, he used it to establish a version of Frobenius descent for arithmetic $\mathscr{D}^{\dagger}$-modules and a comparison result for de Rham complexes (cf. 8] 4.2.4 and 4.3.5). The above results can be viewed as a counterpart of Berthelot's results for convergent isocrystals without assuming a lifting of Frobenius morphism.

Our main result is the following.

Theorem 1.9 (9.3). Let $g: X \rightarrow Y$ be a smooth proper morphism of $k$-schemes locally of finite type. The higher direct image of a convergent isocrystal (resp. F-isocrystal) on $\operatorname{Conv}(X / \mathrm{W})$ (1.3) is a convergent isocrystal (resp. F-isocrystal) on $\operatorname{Conv}(Y / \mathrm{W})$.

In [26], Ogus describle $\mathrm{R}^{i} g_{\mathrm{conv} *}\left(\mathscr{O}_{X / K}\right)$ in terms of relative crystalline cohomology. Recently, Morrow show that it is same as the higher direct images in crystalline cohomology when $Y$ is smooth [25]. Our proof follows a similar line of their work.

1.10. In the following, we explain the structure of this article and the strategy for proving [1.9.

Section 2 contains general conventions and a review on formal geometry. In section 3, we review (iso-)crystals on crystalline site and the finiteness, base change property of their crystalline cohomology due to Shiho [31]. In section 4, we recall the definition of the convergent topos over a $p$-adic base following Shiho [31. In section [5, we show that under a smooth proper morphism of smooth $k$-schemes $X \rightarrow Y$, the higher direct image of a convergent isocrystal on $\operatorname{Conv}(X / \mathrm{W})$ is a " $p$-adic convergent isocrystal" on $\operatorname{Conv}(Y / \mathrm{W})$, i.e. it satisfies the property of coherent crystal in a certain subcategory of $\operatorname{Conv}(Y / \mathrm{W})$ (5.10). Section 6 is devoted to the Frobenius descent (1.5, 1.6). Using Dworks' trick, we deduce 1.9 in the case where $Y$ is smooth over $k$ (6.10). In section 7. we briefly review Raynaud's approach to rigid geometry following Abbes' book [1]. Section 8 is 
DAXIN XU

devoted to a modification of convergent topos which allows us to apply the faithfully flat descent in rigid geometry in the full extent. Based on previous results and Ogus' proper surjective descent, we complete the proof of 1.9 in section 9 .

Acknowledgement. I would like to thank Ahmed Abbes for discussions and his comments on an earlier version of this paper. I would like to thank Atsushi Shiho for his comments and suggestions to improve 1.9. I would like to thank Arthur Ogus for helpful discussions. Part of the work was done when the author was at the BICMR and IHÉS and the author would like to thank their hospitality.

\section{Preliminary}

2.1. In this article, $p$ denotes a prime number, $k$ denotes a perfect field of characteristic $p, \mathrm{~W}$ the ring of Witt vectors of $k$.

We denote by $\mathbf{S}$ the category whose objects are formal W-scheme of finite type ([1] 2.3.13) and morphisms are adic morphisms ([1 2.2.7). By ([22] 6.1.5(v)), morphisms of $\mathbf{S}$ are of finite type. We denote by $\mathbf{S}^{\diamond}$ the full subcategory of $\mathbf{S}$ consisting of flat formal W-schemes of finite type.

Let $\mathfrak{X}$ be a $p$-adic formal $\mathrm{W}$-scheme. For any $n \geq 1$, we denote by $\mathfrak{X}_{n}$ the reduction modulo $p^{n}$ of $\mathfrak{X}$. If we use a gothic letter $\mathfrak{X}$ to denote a $p$-adic formal $\mathrm{W}$-scheme, the corresponding roman letter $X$ will denote its special fiber $\mathfrak{X}_{1}$.

2.2. Let $\mathscr{A}$ be an abelian category. We denote by $\mathscr{A}_{\mathbb{Q}}$ the category with same objects as $\mathscr{A}$ such that the set of morphisms is given for any object $M, N$ of $\mathscr{A}$, by

$$
\operatorname{Hom}_{\mathscr{A}}(M, N)=\operatorname{Hom}_{\mathscr{A}}(M, N) \otimes_{\mathbb{Z}} \mathbb{Q} \text {. }
$$

For any object $M$ of $\mathscr{A}$, we denote its image in $\mathscr{A}_{\mathbb{Q}}$ by $M_{\mathbb{Q}}$.

Definition 2.3 ([10] I 1.3.1). Let $(\mathscr{T}, A)$ be a ringed topos. We say that an $A$-module $M$ of $\mathscr{T}$ is locally projective of finite type if the following equivalent conditions are satisfied:

(i) $M$ is of finite type and the functor $\mathscr{H}_{o m_{A}}(M,-)$ is exact;

(ii) $M$ is of finite type and every epimorphism of $A$-modules $N \rightarrow M$ admits locally a section.

(iii) $M$ is locally a direct summand of a free $A$-module of finite type.

When $\mathscr{T}$ has enough points, and for every point $x$ of $\mathscr{T}$, the stalk of $A$ at $x$ is a local ring, the locally projective $A$-modules of finite type are locally free $A$-modules of finite type ([10] I 2.15.1).

2.4. In the following of this section, $\mathfrak{X}$ denotes an object of $\mathbf{S}(2.1)$. For any $\mathscr{O}_{\mathfrak{X}}$-module $\mathscr{F}$, we set

$$
\mathscr{F}\left[\frac{1}{p}\right]=\mathscr{F} \otimes_{\mathbb{Z}_{p}} \mathbb{Q}_{p}
$$

We denote by $\operatorname{Coh}\left(\mathscr{O}_{\mathfrak{X}}\right)$ (resp. $\left.\operatorname{Coh}\left(\mathscr{O}_{\mathfrak{X}}\left[\frac{1}{p}\right]\right)\right)$ the category of coherent $\mathscr{O}_{\mathfrak{X}}$-modules (resp. $\mathscr{O}_{\mathfrak{X}}\left[\frac{1}{p}\right]$ modules). The canonical functor $\mathbf{C o h}\left(\mathscr{O}_{\mathfrak{X}}\right) \rightarrow \operatorname{Coh}\left(\mathscr{O}_{\mathfrak{X}}\left[\frac{1}{p}\right]\right)$ defined by $\mathscr{F} \mapsto \mathscr{F}\left[\frac{1}{p}\right]$ induces an equivalence of categories ([2] III.6.16)

$$
\operatorname{Coh}\left(\mathscr{O}_{\mathfrak{X}}\right)_{\mathbb{Q}} \stackrel{\sim}{\rightarrow} \operatorname{Coh}\left(\mathscr{O}_{\mathfrak{X}}\left[\frac{1}{p}\right]\right) .
$$

If $\mathfrak{X}=\operatorname{Spf}(A)$ is moreover affine, a coherent $\mathscr{O}_{\mathfrak{X}}\left[\frac{1}{p}\right]$-module $\mathscr{F}$ is locally projective of finite type if and only if $\Gamma(\mathfrak{X}, \mathscr{F})$ is a projective $A\left[\frac{1}{p}\right]$-module of finite type ([2] III.6.17). 
2.5. We denote by $\mathbf{Z a r}_{/ \mathfrak{X}}$ (resp. $\mathfrak{X}_{\text {zar }}$ ) the Zariski site (resp. topos) of $\mathfrak{X}$.

Recall that a family of morphisms of schemes $\left\{f_{i}: T_{i} \rightarrow T\right\}_{i \in I}$ is called an fppf covering if each morphism $f_{i}$ is flat and locally of finite presentation and if $|T|=\bigcup_{i \in I} f_{i}\left(\left|T_{i}\right|\right)$ (cf. [16] IV 6.3.2).

We say that a family of morphisms $\left\{f_{i}: \mathfrak{X}_{i} \rightarrow \mathfrak{X}\right\}_{i \in I}$ of $\mathbf{S}$ is an fppf covering if each morphism $f_{i}$ is flat and if $|\mathfrak{X}|=\bigcup_{i \in I} f_{i}\left(\left|\mathfrak{X}_{i}\right|\right)$. Since $\mathfrak{X}$ is quasi-compact, each fppf covering of $\mathfrak{X}$ admits a finite fppf sub-covering of $\mathfrak{X}$.

By (1] 2.3.16, 5.1.2), a family of adic formal $\mathfrak{X}$-schemes $\left\{\mathfrak{X}_{i} \rightarrow \mathfrak{X}\right\}_{i \in I}$ is an fppf covering if and only if the family $\left\{\mathfrak{X}_{i, n} \rightarrow \mathfrak{X}_{n}\right\}_{i \in I}$ is an fppf covering of schemes for all integer $n \geq 1$.

Since fppf coverings of schemes are stable by base change and by composition, the same holds for fppf coverings of formal $\mathrm{W}$-schemes of finite type.

We denote by $\mathbf{F f t} / \mathfrak{X}$ the full subcategory of $\mathbf{S}_{/ \mathfrak{X}}$ consisting of flat adic formal $\mathfrak{X}$-schemes and by $\mathfrak{X}_{\mathrm{fppf}}$ the topos of sheaves of sets on $\mathbf{F f t} / \mathfrak{X}$, equipped with the topology generated by fppf coverings.

2.6. The canonical functor $\mathbf{Z a r}_{/ \mathfrak{X}} \rightarrow \mathbf{F f t} / \mathfrak{X}$ is continuous and left exact. Then it induces a morphism of topoi

$$
\alpha_{\mathfrak{X}}: \mathfrak{X}_{\mathrm{fppf}} \rightarrow \mathfrak{X}_{\text {zar }} .
$$

Given a morphism $f: \mathfrak{X}^{\prime} \rightarrow \mathfrak{X}$ of $\mathbf{S}$, the canonical functor $\mathbf{F f t} / \mathfrak{X} \rightarrow \mathbf{F f t}_{/ \mathfrak{X}^{\prime}}\left(\right.$ resp. $\mathbf{Z a r}_{/ \mathfrak{X}} \rightarrow$ $\operatorname{Zar}_{/ \mathfrak{X}^{\prime}}$ ) defined by $\mathfrak{Y} \mapsto \mathfrak{Y} \times \mathfrak{X} \mathfrak{X}^{\prime}$ is continuous and left exact. For $\tau \in\{$ zar, fppf $\}$, it induces morphisms of topoi

$$
f_{\tau}: \mathfrak{X}_{\tau}^{\prime} \rightarrow \mathfrak{X}_{\tau}
$$

compatible with $\alpha_{\mathfrak{X}}$ and $\alpha_{\mathfrak{X}^{\prime}}$. If $f$ is a morphism of $\mathbf{F f t} / \mathfrak{X}($ resp. Zar $/ \mathfrak{X})$, in view of the description of direct image functors, one verifies that the above morphism coincides with the localization morphism at $\mathfrak{X}^{\prime}$.

2.7. We say that a closed formal sub-scheme $\mathscr{P}$ of $\mathfrak{X}$ is a rigid point if $\mathscr{P}$ is affine and if $\Gamma(\mathscr{P}, \mathscr{O} \mathscr{P})$ is an 1-valuative order ([1] 1.11.1, 3.3.1). We denote by $\langle\mathfrak{X}\rangle$ the set of rigid points of $\mathfrak{X}$.

Let $f: \mathfrak{X} \rightarrow \mathfrak{Y}$ be a morphism of $\mathbf{S}$. Recall that $f$ is faithfully rig-flat ([1 5.5.9) if it is rig-flat ([1] 5.4.5) and if the associated map $\langle\mathfrak{X}\rangle \rightarrow\langle\mathfrak{Y}\rangle$ on rigid points is surjective.

If $f$ is flat (resp. faithfully flat), then it is rig-flat (resp. faithfully rig-flat) ([1] 5.5.10).

Theorem 2.8 (Gabber, Bosch and Görtz [12]; [1 5.11.11). Let $f: \mathfrak{X}^{\prime} \rightarrow \mathfrak{X}$ be a faithfully rigflat morphism of $\mathbf{S}$. Then, the canonical functor from $\mathbf{C o h}\left(\mathscr{O}_{\mathfrak{X}}\left[\frac{1}{p}\right]\right)$ to the category of coherent $\mathscr{O}_{\mathfrak{X}^{\prime}}\left[\frac{1}{p}\right]$-modules endowed with a descent data in $\operatorname{Coh}\left(\mathscr{O}_{\mathfrak{X}^{\prime} \times \mathfrak{X}^{\prime}}\left[\frac{1}{p}\right]\right)$ is an equivalence.

Although ([1 5.11.11) is an assertion for coherent modules on rigid spaces, the same argument works for coherent $\mathscr{O}_{\mathfrak{X}}\left[\frac{1}{p}\right]$-modules.

Let $\mathscr{U}=\left\{\mathfrak{X}_{i} \rightarrow \mathfrak{X}\right\}_{i \in I}$ be a fppf covering. Since each fppf covering of $\mathfrak{X}$ admits a finite fppf sub-covering of $\mathfrak{X}$, we deduce that any descent datum on coherent $\mathscr{O}_{\mathfrak{X}_{i}}\left[\frac{1}{p}\right]$-modules for $\mathscr{U}$ is effective. The functor from $\mathbf{C o h}\left(\mathscr{O}_{\mathfrak{X}}\left[\frac{1}{p}\right]\right)$ to the category of descent data with respect to $\mathscr{U}$ is fully faithful.

2.9. Let $\mathscr{F}$ be a coherent $\mathscr{O}_{\mathfrak{X}}\left[\frac{1}{p}\right]$-module. By fppf descent for coherent $\mathscr{O}_{\mathfrak{X}}\left[\frac{1}{p}\right]$-modules (2.8), the presheaf on $\mathbf{F f t} / \mathfrak{X}$

$$
\left(f: \mathfrak{X}^{\prime} \rightarrow \mathfrak{X}\right) \mapsto \Gamma\left(\mathfrak{X}^{\prime}, f_{\text {zar }}^{*}(\mathscr{F})\right)
$$

is a sheaf for the fppf topology. In particular, $\mathscr{O}_{\mathfrak{X}}\left[\frac{1}{p}\right]$ defines a sheaf of rings of $\mathfrak{X}_{\mathrm{fppf}}$ that we still denote by $\mathscr{O}_{\mathfrak{X}}\left[\frac{1}{p}\right]$. We call abusively coherent $\mathscr{O}_{\mathfrak{X}}\left[\frac{1}{p}\right]$-module of $\mathfrak{X}_{\mathrm{fppf}}$ a sheaf of $\mathfrak{X}_{\mathrm{fppf}}$ associated to a coherent $\mathscr{O}_{\mathfrak{X}}\left[\frac{1}{p}\right]$-module. 
For $\tau \in\{$ zar,fppf $\}$, the morphism of topoi $f_{\tau}(2.6 .2)$ is ringed by $\mathscr{O}_{\mathfrak{X}}\left[\frac{1}{p}\right]$ and $\mathscr{O}_{\mathfrak{X}^{\prime}}\left[\frac{1}{p}\right]$. For any $\mathscr{O}_{\mathfrak{X}}\left[\frac{1}{p}\right]$-module $M$ of $\mathfrak{X}_{\tau}$, we use $f_{\tau}^{-1}(M)$ to denote the inverse image in the sense of sheaves and we keep $f_{\tau}^{*}(M)$ for the inverse image in the sense of modules.

2.10. Let $\mathscr{A}$ be an open ideal of finite type of $\mathfrak{X}$ ([1 2.1.19). We denote by $\mathfrak{X}^{\prime}$ the admissible blow-up of $\mathscr{A}$ in $\mathfrak{X}$ ([1] 3.1.2). The canonical map $\mathfrak{X}^{\prime} \rightarrow \mathfrak{X}$ is of finite type ([1] 2.3.13) and rig-flat ([1] 5.4.12).

Suppose that $\mathfrak{X}$ is flat over $\mathrm{W}$ and that $\mathscr{A}$ contains $p$. The ideal $\mathscr{A} \mathscr{O}_{\mathfrak{X}^{\prime}}$ is invertible ([1] 3.1.4(i)), and $\mathfrak{X}^{\prime}$ is flat over W ([1] 3.1.4(ii)). We denote by $\mathfrak{X}_{(\mathscr{A} / p)}$ the maximal open formal subscheme of $\mathfrak{X}^{\prime}$ on which

$$
\left(\mathscr{A} \mathscr{O}_{\mathfrak{X}^{\prime}}\right)\left|\mathfrak{X}_{(\mathscr{A} / p)}=\left(p \mathscr{O}_{\mathfrak{X}^{\prime}}\right)\right| \mathfrak{X}_{(\mathscr{A} / p)}
$$

and we call it the dilatation of $\mathscr{A}$ with respect to $p$ ([1] 3.2.3.4 and 3.2.7). Note that $\mathfrak{X}_{(\mathscr{A} / p)}$ is the complement of $\operatorname{Supp}\left(\mathscr{A} \mathscr{O}_{\mathfrak{X}^{\prime}} / p \mathscr{O}_{\mathfrak{X}^{\prime}}\right)$ in $\mathfrak{X}^{\prime}([22] 0.5 .2 .2)$.

Let $S$ be a closed subscheme of $X$ and $\mathscr{I}$ the ideal sheaf associated to the canonical morphism $S \rightarrow \mathfrak{X}$. For any $n \geq 1$, we denote by $\mathfrak{T}_{S, n}(\mathfrak{X})$ the dilatation of $\mathscr{I}^{n}+p \mathscr{O}_{\mathfrak{X}}$ with respect to $p([26]$ 2.5) 2. By (2.10.1), there exists a morphism from the reduced subscheme of $\left(\mathfrak{T}_{S, n}(\mathfrak{X})\right)_{1}$ to $S$ which fits into the following diagram:

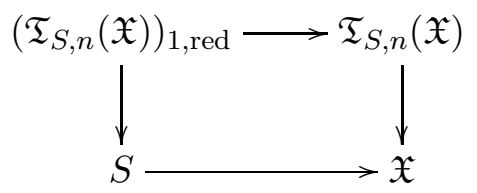

The universal property of dilatation ([1] 3.2.6) can be reinterpreted in the following way.

Proposition 2.11 ([34] 3.6, 3.10). Keep the notations and assumptions of 2.10, Let $\mathfrak{T}$ be an adic flat formal W-scheme, $\underline{T}$ the closed subscheme of $T$ defined by the ideal sheaf $\left\{x \in \mathscr{O}_{T} \mid x^{p}=0\right\}$ and $f: \mathfrak{T} \rightarrow \mathfrak{X}$ an adic morphism. Suppose that there exists a morphism $T \rightarrow S$ (resp. $\underline{T} \rightarrow S$ ) which fits into the following diagram
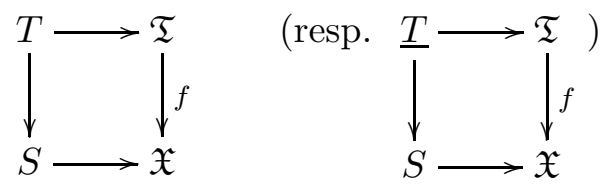

Then there exists a unique adic morphism $g: \mathfrak{T} \rightarrow \mathfrak{T}_{S, 1}(\mathfrak{X})$ (resp. $g: \mathfrak{T} \rightarrow \mathfrak{T}_{S, p}(\mathfrak{X})$ ) lifting $f$.

2.12. Let $\mathscr{C}$ and $\mathscr{D}$ be two categories, $\widehat{\mathscr{C}}$ (resp. $\widehat{\mathscr{D}}$ ) the category of presheaves of sets on $\mathscr{C}$ (resp. $\mathscr{D})$ and $u: \mathscr{C} \rightarrow \mathscr{D}$ a functor. We have a functor

$$
\widehat{u}^{*}: \widehat{\mathscr{D}} \rightarrow \widehat{\mathscr{C}} \quad \mathscr{G} \mapsto \widehat{u}^{*}(\mathscr{G})=\mathscr{G} \circ u .
$$

It admits a right adjoint ([3] I 5.1)

$$
\widehat{u}_{*}: \widehat{\mathscr{C}} \rightarrow \widehat{\mathscr{D}}
$$

\footnotetext{
${ }^{2}$ The union of rigid space $\cup_{n \geq 1} \mathfrak{T}_{S, n}(\mathfrak{X})^{\text {rig }}$ is same as the tube of $S$ in $\mathfrak{X}$ introduced by Berthelot (cf. [6] 1.1.2, 1.1.10).
} 
Let $\mathscr{C}$ and $\mathscr{D}$ be two sites $\mathbb{3}^{3}$. If $u: \mathscr{C} \rightarrow \mathscr{D}$ is a cocontinuous (resp. continuous) functor and $\mathscr{F}$ (resp. $\mathscr{G})$ is a sheaf on $\mathscr{C}($ resp. $\mathscr{D})$, then $\widehat{u}_{*}(\mathscr{F})\left(\right.$ resp. $\left.\widehat{u}^{*}(\mathscr{G})\right)$ is a sheaf on $\mathscr{D}$ (resp. $\left.\mathscr{C}\right)$ ([3] III $1.2,2.2)$.

Let $\tilde{\mathscr{C}}$ (resp. $\widetilde{\mathscr{D}}$ ) be the topos of the sheaves of sets on $\mathscr{C}$ (resp. $\mathscr{D}$ ) and $u: \mathscr{C} \rightarrow \mathscr{D}$ a cocontinuous functor. Then $u$ induces a morphism of topoi $g: \widetilde{\mathscr{C}} \rightarrow \widetilde{\mathscr{D}}$ defined by $g_{*}=\widehat{u}_{*}$ and $g^{*}=a \circ \widehat{u}^{*}$, where $a$ is the sheafification functor (cf. [3] III 2.3).

Proposition 2.13 (29] 4.2.1). Let $\mathscr{C}$ be a site, $\mathscr{D}$ a site whose topology is defined by a pretopology and $u: \mathscr{C} \rightarrow \mathscr{D}$ a functor. Assume that:

(i) $u$ is fully faithful,

(ii) $u$ is continuous and cocontinuous,

(iii) For every object $V$ of $\mathscr{D}$, there exists a covering of $V$ in $\mathscr{D}$ of the form $\left\{u\left(U_{i}\right) \rightarrow V\right\}_{i \in I}$ where $U_{i}$ is an object of $\mathscr{C}$.

Then the morphism of topoi $g: \tilde{\mathscr{C}} \rightarrow \widetilde{\mathscr{D}}$ defined by $g^{*}=\widehat{u}^{*}$ and $g_{*}=\widehat{u}_{*}(2.12)$ is an equivalence of topoi.

\section{Crystalline COHOMOlOgy For isocrystals}

3.1. In this section, $\mathfrak{S}$ denotes a flat formal $\mathrm{W}$-scheme of finite type and $X$ an $S$-scheme.

We equip $p \mathscr{O}_{\mathfrak{S}}$ with the canonical PD-structure $\gamma$. Recall that the crystalline site Crys $(X / \mathfrak{S})$ is defined as follows ([1] 7.17): an object is a quadraple $(U, T, \iota, \delta)$ consisting of an open subscheme $U$ of $X$, a scheme $T$ over $\mathfrak{S}_{n}$ for some integer $n \geq 1$, a closed immersion $\iota: U \rightarrow T$ and a PDstructure $\delta$ on $\operatorname{Ker}\left(\mathscr{O}_{T} \rightarrow \mathscr{O}_{U}\right)$ compatible with $\gamma$. A morphism from $\left(U^{\prime}, T^{\prime}, \iota^{\prime}, \delta^{\prime}\right)$ to $(U, T, \iota, \delta)$ of $\operatorname{Crys}(X / \mathfrak{S})$ consists of an open immersion $U^{\prime} \rightarrow U$ and an $\mathfrak{S}$-morphism $T^{\prime} \rightarrow T$ compatible with $\iota^{\prime}, \iota$ and the PD-structures. A family of morphisms $\left\{\left(U_{i}, T_{i}\right) \rightarrow(U, T)\right\}_{i \in I}$ is a covering if each morphism $T_{i} \rightarrow T$ is an open immersion and $|T|=\bigcup_{i \in I}\left|T_{i}\right|$. We denote by $(X / \mathfrak{S})_{\text {crys }}$ the topos of sheaves of sets on $\operatorname{Crys}(X / \mathfrak{S})$.

The presheaf of rings defined by $(U, T) \mapsto \Gamma\left(T, \mathscr{O}_{T}\right)$, is a sheaf that we denote by $\mathscr{O}_{X / \mathfrak{S}}^{\text {crys }}$. For

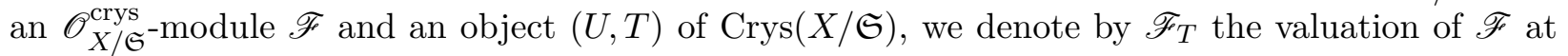
$(U, T)([1] 5.1)$.


morphism $f:\left(U^{\prime}, T^{\prime}\right) \rightarrow(U, T)$ of $\operatorname{Crys}(X / \mathfrak{S})$, the transition morphism $f^{*}\left(\mathscr{F}_{T}\right) \rightarrow \mathscr{F}_{T^{\prime}}$ is an isomorphism.


$\mathscr{O}_{T}$-module of finite presentation for every object $(U, T)$ of $\operatorname{Crys}(X / \mathfrak{S})$.

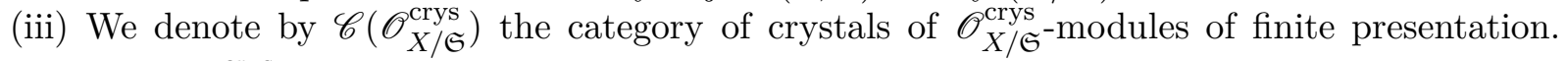
Objects of $\mathscr{C}\left(\mathscr{O}_{X / \mathfrak{S}}^{\text {crys }}\right) \mathbb{Q}(2.2)$ are called isocrystals.

3.3. Crystals have an equivalent description in terms of modules equppied with hyper-PD-stratification and of modules with integrable connection. Let us briefly recall these notions.

Let $\mathfrak{X}$ be an adic formal $\mathfrak{S}$-scheme of finite type. For any integer $r \geq 1$, let $\mathfrak{X}^{r+1}$ be the fiber product of $(r+1)$-copies of $\mathfrak{X}$ over $\mathfrak{S}$.

\footnotetext{
${ }^{3}$ We suppose that the site $\mathscr{C}$ is small.
} 
Let $\mathrm{G}$ be an adic formal $\mathfrak{X}^{2}$-scheme and let $q_{1}, q_{2}: \mathrm{G} \rightarrow \mathfrak{X}$ be the canonical projections. A formal $\mathfrak{X}$-groupoid struture over $\mathfrak{S}$ on $\mathrm{G}$ are three adic morphisms

$$
\alpha: \mathrm{G} \times \mathfrak{X} \mathrm{G} \rightarrow \mathrm{G}, \quad \iota: \mathfrak{X} \rightarrow \mathrm{G}, \quad \eta: \mathrm{G} \rightarrow \mathrm{G}
$$

where the fibered product $\mathrm{G} \times \mathfrak{X} \mathrm{G}$ is taken on the left (resp. right) for the $\mathfrak{X}$-structure defined by $q_{2}$ (resp. $q_{1}$ ), satisfying the compatibility conditions for groupoid (cf. [34] 4.8). We set $q_{13}=\alpha$ and $q_{12}, q_{23}: \mathrm{G} \times \mathfrak{X} \mathrm{G} \rightarrow \mathrm{G}$ the projection in the first and second component respectively.

Definition 3.4. Let $\mathrm{G}$ be a formal $\mathfrak{X}$-groupoid over $\mathfrak{S}$ and $M$ an $\mathscr{O}_{\mathfrak{X}}$-module. An $\mathscr{O}_{\mathrm{G}}$-stratification on $M$ is an $\mathscr{O}_{\mathrm{G}}$-linear isomorphism

$$
\varepsilon: q_{2}^{*}(M) \stackrel{\sim}{\rightarrow} q_{1}^{*}(M)
$$

satisfying $\iota^{*}(\varepsilon)=\mathrm{id}_{M}$ and the cocycle condition $q_{12}^{*}(\varepsilon) \circ q_{23}^{*}(\varepsilon)=q_{13}^{*}(\varepsilon)$.

3.5. Let $f: \mathfrak{X} \rightarrow \mathfrak{S}$ be a smooth morphism, $M$ a coherent $\mathscr{O}_{\mathfrak{X}}$-module (resp. $\mathscr{O}_{\mathfrak{X}}\left[\frac{1}{p}\right]$-module) and $\widehat{\Omega}_{\mathfrak{X} / \mathfrak{S}}^{1}$ the $\mathscr{O}_{\mathfrak{X}}$-module of differentials of $\mathfrak{X}$ relative to $\mathfrak{S}$. A connection on $M$ relative to $\mathfrak{S}$ is an $f^{-1}\left(\mathscr{O}_{\mathfrak{S}}\right)$-linear morphism

$$
\nabla: M \rightarrow M \otimes_{\mathscr{O}_{\mathfrak{X}}} \widehat{\Omega}_{\mathfrak{X} / \mathfrak{S}}^{1}
$$

such that for every local sections $f$ of $\mathscr{O}_{\mathfrak{X}}$ and $e$ of $M$, we have $\nabla(f e)=e \otimes d(f)+f \nabla(e)$.

For any $q \geq 0$, the morphism $\nabla$ extends to a unique $f^{-1}\left(\mathscr{O}_{\mathfrak{S}}\right)$-linear morphism

$$
\nabla_{q}: M \otimes_{\mathscr{O}_{\mathfrak{X}}} \widehat{\Omega}_{\mathfrak{X} / \mathfrak{S}}^{q} \rightarrow M \otimes_{\mathscr{O}_{\mathfrak{X}}} \widehat{\Omega}_{\mathfrak{X} / \mathfrak{S}}^{q+1} .
$$

The composition $\nabla_{1} \circ \nabla$ is $\mathscr{O}_{\mathfrak{X}}$-linear. We say that $\nabla$ is integrable if $\nabla_{1} \circ \nabla=0$. If $\nabla$ is integrable, we have $\nabla_{q+1} \circ \nabla_{q}=0$ for all $q \geq 0$ and we can associate to $(M, \nabla)$ a de Rham complex

$$
M \stackrel{\nabla}{\rightarrow} M \otimes_{\mathscr{O}_{\mathfrak{X}}} \widehat{\Omega}_{\mathfrak{X} / \mathfrak{S}}^{1} \stackrel{\nabla_{1}}{\longrightarrow} M \otimes_{\mathscr{O}_{\mathfrak{X}}} \widehat{\Omega}_{\mathfrak{X} / \mathfrak{S}}^{2} \stackrel{\nabla_{2}}{\longrightarrow} \cdots .
$$

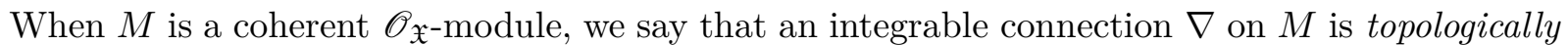
quasi-nilpotent if for every $n \geq 1$, its reduction modulo $p^{n}$ on $M / p^{n} M$ is quasi-nilpotent ([1] 6.1).

3.6. Suppose that $X$ is smooth over $S$ and admits a smooth lifting $\mathfrak{X}$ over $\mathfrak{S}$. We denote by $\mathrm{P}_{\mathfrak{X}_{n} / \mathfrak{S}_{n}}$ the PD-envelop of the diagonal immersion $\mathfrak{X}_{n} \rightarrow \mathfrak{X}_{n}^{2}$ compatible with the canonical PD-structure $\gamma$ (3.1) for $n \geq 1$. The sequence $\left(\mathrm{P}_{\mathfrak{X}_{n} / \mathfrak{S}_{n}}\right)_{n \geq 1}$ forms an adic inductive $\left(\mathfrak{X}_{n}^{2}\right)_{n \geq 1}$-system (cf. [11] 3.20.8)

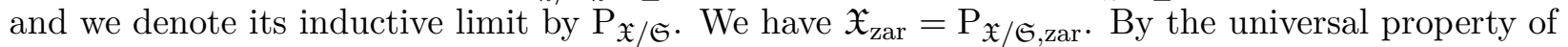
PD-envelope, the formal $\mathfrak{X}^{2}$-schema $\mathrm{P}_{\mathfrak{X} / \mathfrak{S}}$ is equipped with a formal $\mathfrak{X}$-groupoid structure (3.3).

Given an object $\mathscr{F}$ of $\mathscr{C}\left(\mathscr{O}_{X / \mathfrak{S}}^{\text {crys }}\right)(\underline{3.2})$, the coherent $\mathscr{O}_{\mathfrak{X}}$-module $\mathscr{F}_{\mathfrak{X}}=\varliminf_{n>1} \mathscr{F}_{\mathfrak{X}_{n}}$ is equipped with an $\mathscr{O}_{\mathrm{P}_{\mathfrak{X} / \mathfrak{S}}}$-stratification and then an integrable connection. Moreover, the following categories are canonically equivalent ([11] 6.6, [35] 07JH):

(i) The category $\mathscr{C}\left(\mathscr{O}_{X / \mathfrak{S}}^{\text {crys }}\right)$.

(ii) The category of coherent $\mathscr{O}_{\mathfrak{X}}$-modules equipped with an $\mathscr{O}_{\mathrm{P}_{\mathfrak{X} / \mathfrak{S}}}$-stratification.

(iii) The category of coherent $\mathscr{O}_{\mathfrak{X}}$-modules equipped with a topologically quasi-nilpotent integrable connection relative to $\mathfrak{S}$.

Proposition 3.7 ([27] 0.7.5, [31] 1.23). Keep the assumption of [3.6. Let $M$ be a coherent $\mathscr{O}_{\mathfrak{X}}\left[\frac{1}{p}\right]$ -

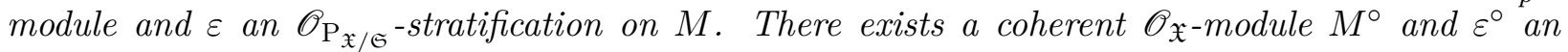
$\mathscr{O}_{\mathrm{P}_{\mathfrak{X} / \mathfrak{G}}}$-stratification on $M^{\circ}$ such that $\left(M^{\circ}\left[\frac{1}{p}\right], \varepsilon^{\circ} \otimes \mathrm{id}\right)$ is isomorphic to $(M, \varepsilon)$. 
Lemma 3.8. Let $\mathfrak{X}$ be a smooth formal $\mathrm{W}$-scheme, $M$ a coherent $\mathscr{O}_{\mathfrak{X}}$-module and $\nabla$ an integrable connection on $M$ relative to $\mathrm{W}$. Then, $M\left[\frac{1}{p}\right]$ is a locally projective $\mathscr{O}_{\mathfrak{X}}\left[\frac{1}{p}\right]$-module of finite type. In particular, given a coherent $\mathscr{O}_{\mathfrak{X}}\left[\frac{1}{p}\right]$-module with an $\mathscr{O}_{\mathrm{P}_{\mathfrak{X} / \mathrm{W}}}$-stratification $(M, \varepsilon)$ (resp. an object $\mathscr{E}$ of $\left.\mathscr{C}\left(\mathscr{O}_{X / \mathrm{W}}^{\mathrm{crys}}\right)\right), M$ (resp. $\left.\mathscr{E}_{\mathfrak{X}}\left[\frac{1}{p}\right]\right)$ is a locally projective $\mathscr{O}_{\mathfrak{X}}\left[\frac{1}{p}\right]$-module of finite type.

The first assertion is a standard result (cf. [20] 8.8, [21] 1.2). Then the second assertion follows from 3.6 and 3.7 .

3.9. There exists a canonical functor $\pi: \operatorname{Crys}(X / \mathfrak{S}) \rightarrow \operatorname{Zar}_{/ X}$ defined by $(U, \mathfrak{T}) \mapsto U$. It is cocontinuous and then induces a morphism of topoi ([11] 5.12)

$$
u_{X / \mathfrak{S}, \text { crys }}:(X / \mathfrak{S})_{\text {crys }} \rightarrow X_{\text {zar }} \text {. }
$$

We denote by $g_{X / \mathfrak{S}, \text { crys }}$ the composition

$$
g_{X / \mathfrak{S}, \text { crys }}:(X / \mathfrak{S})_{\text {crys }} \rightarrow X_{\text {zar }} \rightarrow \mathfrak{S}_{\text {zar }}
$$

which is ringed by $\mathscr{O}_{X / \mathfrak{S}}^{\text {crys }}$ and $\mathscr{O}_{\mathfrak{S}}$. We call $\mathrm{R}^{\bullet} g_{X / \mathfrak{S} \text {,crys* }}(-)$ the relative crystalline cohomology.

For an isocrystal $\mathscr{E}=\mathscr{F}_{\mathbb{Q}}$ with $\mathscr{F} \in \mathbf{O b}\left(\mathscr{C}\left(\mathscr{O}_{X / \mathfrak{S}}^{\text {crys }}\right)\right)$, we set (2.4.1)

$$
\mathrm{R}^{q} g_{X / \mathfrak{S}, \operatorname{crys} *}(\mathscr{E})=\mathrm{R}^{q} g_{X / \mathfrak{S}, \operatorname{crys} *}(\mathscr{F})\left[\frac{1}{p}\right], \quad \mathrm{R} g_{X / \mathfrak{S}, \operatorname{crys} *}(\mathscr{E})=\mathrm{R} g_{X / \mathfrak{S}, \operatorname{crys} *}(\mathscr{F})\left[\frac{1}{p}\right] .
$$

It is clear that the above definition is independent of the choice of $\mathscr{F}$.

Theorem 3.10 ([31] 1.15). Suppose moreover that $\mathfrak{S}$ is separated and that $X$ is smooth and proper over $S$. Then, for any isocrystal $\mathscr{E}$ and $q \geq 0$, the relative crystalline cohomology $\mathrm{R}^{q} g_{X / \mathfrak{S}, \text { crys } *}(\mathscr{E})$ is a coherent $\mathscr{O}_{\mathfrak{S}}\left[\frac{1}{p}\right]$-module.

Definition 3.11 (31] 1.2, 1.9). Let $\mathfrak{T}$ be an adic formal $\mathrm{W}$-scheme.

(i) We denote by $\mathrm{M}(\mathfrak{T})$ the category of projective systems of $\left(\mathscr{O}_{\mathfrak{T}_{n}}\right)_{n \geq 1}$-modules $\left(M_{n}\right)_{n \geq 1}$ such that each $M_{n}$ is an $\mathscr{O}_{\mathfrak{T}_{n}}$-module of finite presentation and that the canonical morphism $M_{n+1} \otimes_{\mathfrak{O}_{\mathfrak{x}_{n+1}}}$ $\mathscr{O}_{\mathfrak{T}_{n}} \stackrel{\sim}{\rightarrow} M_{n}$ is an isomorphism.

(ii) We say that an object $M$ of $\mathrm{M}(\mathfrak{T})_{\mathbb{Q}}(2.2)$ is locally projective if Zariski locally on $\mathfrak{T}, M$ is isomorphic to a direct summand of $\left(\left(\mathscr{O}_{\mathfrak{T}_{n}}^{\oplus r}\right)_{n \geq 1}\right)_{\mathbb{Q}}$ for some integer $r$.

If $\mathfrak{T}$ is of finite type over $\mathrm{W}$, the canonical functor $\left(M_{n}\right)_{n \geq 1} \mapsto \lim _{n \geq 1} M_{n}$ induces an equivalence of categories $\mathrm{M}(\mathfrak{T}) \stackrel{\sim}{\rightarrow} \operatorname{Coh}\left(\mathscr{O}_{\mathfrak{T}}\right)$. In this case, an object of $\mathrm{M}(\mathfrak{T})_{\mathbb{Q}}$ is locally projective (3.11) if and only if its image in $\operatorname{Coh}\left(\mathscr{O}_{\mathfrak{T}}\left[\frac{1}{p}\right]\right)(2.4 .2)$ is locally projective of finite type (2.3).

Definition $3.12(31] 1.11,1.12)$. (i) A projective system of objects $\left\{\left(U, T_{n}, \iota_{n}, \delta_{n}\right)\right\}_{n \geq 1}$ of $\operatorname{Crys}(X / \mathfrak{S})$ is called a p-adic system, if $\mathfrak{T}:=\lim _{n} T_{n}$ is an adic formal $\mathfrak{S}$-scheme such that the canonical morphism $T_{n} \rightarrow \mathfrak{T} \otimes_{\mathbb{Z}_{p}} \mathbb{Z} / p^{n} \mathbb{Z}$ is an isomorphism.

(ii) We say that an isocrystal $\mathscr{E}=\mathscr{F}_{\mathbb{Q}}(3.2)$ is locally projective if for every $p$-adic system $\mathfrak{T}=\left(U, \mathfrak{T}_{n}\right)_{n \geq 1}$ of $\operatorname{Crys}(X / \mathfrak{S}),\left(\left(\mathscr{F}_{\mathfrak{T}_{n}}\right)_{n \geq 1}\right)_{\mathbb{Q}} \in \mathrm{M}(\mathfrak{T})_{\mathbb{Q}}$ is locally projective in the sense of 3.11 .

Theorem 3.13 ([31] 1.16). Keep the assumption of [3.10. For any locally projective isocrystal $\mathscr{E}$, $\mathrm{R} g_{X / \mathfrak{S}, \text { crys } *}(\mathscr{E})$ is a perfect complex of $\mathscr{O}_{\mathfrak{S}}\left[\frac{1}{p}\right]$-modules, i.e. Zariski locally on $\mathfrak{S}$, it is isomorphic to a bounded complex of locally projective $\mathscr{O}_{\mathfrak{S}}\left[\frac{1}{p}\right]$-modules of finite type in the derived category of $\mathscr{O}_{\mathfrak{S}}\left[\frac{1}{p}\right]$-modules. 
Theorem 3.14 (31] 1.19). Let $\varphi: \mathfrak{S}^{\prime} \rightarrow \mathfrak{S}$ be an adic morphism of seperated, flat formal Wschemes of finite type, $X$ a smooth proper $S$-scheme, $X^{\prime}=X \times_{\mathfrak{S}} \mathfrak{S}^{\prime}$ and $\varphi_{\text {crys }}:\left(X^{\prime} / \mathfrak{S}^{\prime}\right)_{\text {crys }} \rightarrow$ $(X / \mathfrak{S})_{\text {crys }}$ the functorial morphism of crystalline topoi. Then, for any locally projective isocrystal $\mathscr{E}$ of $(X / \mathfrak{S})_{\text {crys }}$, there exists a canonical isomorphism in the derived category of $\mathscr{O}_{\mathfrak{S}^{\prime}}\left[\frac{1}{p}\right]$-modules:

$$
\mathrm{L} \varphi_{\text {zar }}^{*}\left(\mathrm{R} g_{X / \mathfrak{S}, \operatorname{crys} *}(\mathscr{E})\right) \stackrel{\sim}{\rightarrow} \mathrm{R} g_{X^{\prime} / \mathfrak{S}^{\prime}, \operatorname{crys} *}\left(\varphi_{\text {crys }}^{*}(\mathscr{E})\right)
$$

\section{Convergent topos And COnvergent isocrystals}

In this section, $\mathfrak{S}$ denotes a flat formal $\mathrm{W}$-scheme of finite type and $X$ denotes an $S$-scheme. For any scheme $T$, we denote by $T_{0}$ the reduced subscheme of $T$.

Definition 4.1 ([26] 2.1, [31] 2.4). We define a category $\operatorname{Conv}(X / \mathfrak{S})$ as follows.

(i) An object of $\operatorname{Conv}(X / \mathfrak{S})$ is a pair $(\mathfrak{T}, u)$ consisting of a formal $\mathfrak{S}$-scheme of finite type which is flat over $\mathrm{W}$ and an $S$-morphism $u: T_{0} \rightarrow X$.

(ii) Let $\left(\mathfrak{T}^{\prime}, u^{\prime}\right)$ and $(\mathfrak{T}, u)$ be two objects of $\operatorname{Conv}(X / \mathfrak{S})$. A morphism from $\left(\mathfrak{T}^{\prime}, u^{\prime}\right)$ to $(\mathfrak{T}, u)$ is a $\mathfrak{S}$-morphism $f: \mathfrak{T}^{\prime} \rightarrow \mathfrak{T}$ such that the induced morphism $f_{0}: T_{0}^{\prime} \rightarrow T_{0}$ is compatible with $u^{\prime}$ and $u$.

We denote an object $(\mathfrak{T}, u)$ of $\operatorname{Conv}(X / \mathfrak{S})$ simply by $\mathfrak{T}$, if there is no risk of confusion.

It is clear that if $X \rightarrow Y$ is a nilpotent immersion of $S$-schemes, the category $\operatorname{Conv}(X / \mathfrak{S})$ is canonically equivalent to $\operatorname{Conv}(Y / \mathfrak{S})$.

4.2. Let $f:\left(\mathfrak{T}^{\prime}, u^{\prime}\right) \rightarrow(\mathfrak{T}, u)$ and $g:\left(\mathfrak{T}^{\prime \prime}, u^{\prime \prime}\right) \rightarrow(\mathfrak{T}, u)$ be two morphisms of $\operatorname{Conv}(X / \mathfrak{S})$. We denote by $\mathfrak{Z}$ the closed formal sub-scheme of $\mathfrak{T}^{\prime} \times_{\mathfrak{T}} \mathfrak{T}^{\prime \prime}$ defined by the ideal of $p$-torsion elements of $\mathscr{O}_{\mathfrak{T}^{\prime} \times \mathfrak{T}^{\prime \prime}}$. The fibered product of $f$ and $g$ in $\operatorname{Conv}(X / \mathfrak{S})$ is represented by $\mathfrak{Z}$, which is flat over $\mathrm{W}$, equipped with the composition $Z_{0} \rightarrow T_{0}^{\prime} \times_{T_{0}} T_{0}^{\prime \prime} \rightarrow X$ induced by $u^{\prime}$ and $u^{\prime \prime}$.

If either $\mathfrak{T}^{\prime \prime} \rightarrow \mathfrak{T}$ or $\mathfrak{T}^{\prime} \rightarrow \mathfrak{T}$ is flat, then $\mathfrak{Z}$ is equal to $\mathfrak{T}^{\prime} \times \mathfrak{T} \mathfrak{T}^{\prime \prime}$.

4.3. We say that a family of morphisms $\left\{\left(\mathfrak{T}_{i}, u_{i}\right) \rightarrow(\mathfrak{T}, u)\right\}_{i \in I}$ is a Zariski (resp. fppf) covering if morphisms of formal schemes $\left\{\mathfrak{T}_{i} \rightarrow \mathfrak{T}\right\}_{i \in I}$ is a Zariski (resp. fppf) covering. By 2.5, Zariski (resp. fppf) coverings form a pretopology. For $\tau \in\{$ zar, fppf $\}$, we denote by $(X / \mathfrak{S})_{\operatorname{conv}, \tau}$ the topos of sheaves of sets on $\operatorname{Conv}(X / \mathfrak{S})$, equipped with the $\tau$-topology.

Remark 4.4. The above definition of convergent site is slightly different to that of [26, 31] where they consider a category whose objects are triples $(\mathfrak{T}, Z, u)$ where $\mathfrak{T}$ is the same as above, $Z$ is a closed subscheme of definition of $\mathfrak{T}$ such that $T_{0} \rightarrow \mathfrak{T}$ factors through $Z$ and $u: Z \rightarrow X$ is an $S$-morphism. However, it follows from 2.13 that the convergent topoi (with Zariski topology) defined by two different ways are equivalent and we freely use results of [31] in our setting.

4.5. Let $(\mathfrak{T}, u)$ be an object of $\operatorname{Conv}(X / \mathfrak{S})$ and $\tau \in\{$ zar, fppf $\}$. The canonical functor

$$
\begin{aligned}
\mathbf{F f t}_{/ \mathfrak{T}}\left(\operatorname{resp} . \mathbf{Z a r}_{/ \mathfrak{T}}\right) & \rightarrow \operatorname{Conv}(X / \mathfrak{S}) \\
\left(f: \mathfrak{T}^{\prime} \rightarrow \mathfrak{T}\right) & \mapsto\left(\mathfrak{T}^{\prime}, u \circ f_{0}\right)
\end{aligned}
$$

is cocontinuous and it induces a morphism of topoi

$$
s_{\mathfrak{T}}: \mathfrak{T}_{\tau} \rightarrow(X / \mathfrak{S})_{\operatorname{conv}, \tau} \quad \tau \in\{\text { zar, fppf }\} .
$$

For any sheaf $\mathscr{F}$ of $(X / \mathfrak{S})_{\text {conv }, \tau}$, we set $\mathscr{F}_{\mathfrak{T}}=s_{\mathfrak{T}}^{*}(\mathscr{F})$. For any morphism $f: \mathfrak{T}^{\prime} \rightarrow \mathfrak{T}$ of $\operatorname{Conv}(X / \mathfrak{S})$, we have a canonical morphism

$$
\beta_{f}: \mathscr{F}_{\mathfrak{T}} \rightarrow f_{\tau *}\left(\mathscr{F}_{\mathfrak{T}^{\prime}}\right)
$$


and we denote its adjoint by

$$
\gamma_{f}: f_{\tau}^{*}\left(\mathscr{F}_{\mathfrak{T}}\right) \rightarrow \mathscr{F}_{\mathfrak{T}^{\prime}}
$$

where $f_{\tau}: \mathfrak{T}_{\tau}^{\prime} \rightarrow \mathfrak{T}_{\tau}$ denotes the functorial morphism for $\tau$-topology (2.6). It is clear that $\gamma_{\text {id }}=\mathrm{id}$. If $f$ is a morphism of $\mathbf{F f t}_{/ \mathfrak{T}}\left(\operatorname{resp} . \mathbf{Z a r}_{/ \mathfrak{T}}\right), f_{\tau}$ is the localisation morphism at $\mathfrak{T}^{\prime}(2.6)$ and then $\gamma_{f}$ is an isomorphism. If $g: \mathfrak{T}^{\prime \prime} \rightarrow \mathfrak{T}^{\prime}$ is another morphism of $\operatorname{Conv}(X / \mathfrak{S})$, one verifies that $\gamma_{g \circ f}=\gamma_{f} \circ f_{\tau}^{*}\left(\gamma_{g}\right)$.

Proposition 4.6. For $\tau \in\{$ zar, fppf $\}$, a sheaf $\mathscr{F}$ of $\operatorname{Conv}(X / \mathfrak{S})_{\operatorname{conv}, \tau}$ is equivalent to the following data:

(i) For every object $(\mathfrak{T}, u)$ of $\operatorname{Conv}(X / \mathfrak{S})$, a sheaf $\mathscr{F}_{\mathfrak{T}}$ of $\mathfrak{T}_{\tau}$,

(ii) For every morphism $f:\left(\mathfrak{T}^{\prime}, u^{\prime}\right) \rightarrow(\mathfrak{T}, u)$, a transition morphism $\gamma_{f}$ (4.5.4),

subject to the following conditions

(a) If $f$ is the identity morphism of $(\mathfrak{T}, u), \gamma_{f}$ is the identity morphism.

(b) If $f: \mathfrak{T}^{\prime} \rightarrow \mathfrak{T}$ is a morphism of $\mathbf{Z a r}_{/ \mathfrak{T}}$ (resp. $\left.\mathbf{F f t} / \mathfrak{T}\right), \gamma_{f}$ is an isomorphism.

(c) If $f$ and $g$ are two composable morphisms, then we have $\gamma_{g \circ f}=\gamma_{f} \circ f_{\tau}^{*}\left(\gamma_{g}\right)$.

Proof. Given a data $\left\{\mathscr{F}_{\mathfrak{T}}, \gamma_{f}\right\}$ as in the proposition, for any morphism $f: \mathfrak{T}^{\prime} \rightarrow \mathfrak{T}$ of $\operatorname{Conv}(X / \mathfrak{S})$, the morphism $\gamma_{f}$ induces a morphism

$$
\mathscr{F}_{\mathfrak{T}}(\mathfrak{T}) \rightarrow \mathscr{F}_{\mathfrak{T}^{\prime}}\left(\mathfrak{T}^{\prime}\right)
$$

In view of conditions (a) and (c), the correspondence

$$
\mathfrak{T} \mapsto \mathscr{F}_{\mathfrak{T}}(\mathfrak{T})
$$

defines a presheaf $\mathscr{F}$ on $\operatorname{Conv}(X / \mathfrak{S})$. In view of condition (b), $\mathscr{F}$ is a sheaf and the above construction is quasi-inverse to 4.5. Then the proposition follows.

4.7. Note that the fppf topology on $\operatorname{Conv}(X / \mathfrak{S})$ is finer than the Zariski topology. Equipped with the fppf topology on the source and the Zariski topology on the target, the identical functor id : $\operatorname{Conv}(X / \mathfrak{S}) \rightarrow \operatorname{Conv}(X / \mathfrak{S})$ is cocontinuous. By 2.12, it induces a morphism of topoi

$$
\alpha:(X / \mathfrak{S})_{\mathrm{conv}, \mathrm{fppf}} \rightarrow(X / \mathfrak{S})_{\mathrm{conv}, \mathrm{zar}} .
$$

If $\mathscr{F}$ is a sheaf of $(X / \mathfrak{S})_{\text {conv,fppf }}, \alpha_{*}(\mathscr{F})$ is equal to $\mathscr{F}$ as presheaves. If $\mathscr{G}$ is a sheaf of $(X / \mathfrak{S})_{\text {conv,zar }}$, then $\alpha^{*}(\mathscr{G})$ is the sheafification of $\mathscr{G}$ with respect to the fppf topology.

4.8. Let $g: \mathfrak{S}^{\prime} \rightarrow \mathfrak{S}$ be a morphism of $\mathbf{S}^{\diamond}$ (2.1), $X^{\prime}$ an $S^{\prime}$-scheme and $f: X^{\prime} \rightarrow X$ a morphism compatible with $g$, i.e. the diagram

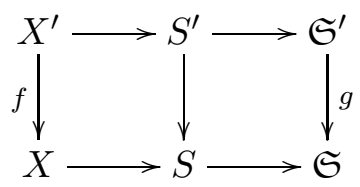

is commutative. For any object $(\mathfrak{T}, u)$ of $\operatorname{Conv}\left(X^{\prime} / \mathfrak{S}^{\prime}\right),(\mathfrak{T}, f \circ u)$ defines an object of $\operatorname{Conv}(X / \mathfrak{S})$. We obtain a functor that we denote by

$$
\varphi: \operatorname{Conv}\left(X^{\prime} / \mathfrak{S}^{\prime}\right) \rightarrow \operatorname{Conv}(X / \mathfrak{S}), \quad(\mathfrak{T}, u) \mapsto(\mathfrak{T}, f \circ u) .
$$

It is clear that $\varphi$ commutes with the fibered product (4.2). 
Lemma 4.9. (i) Let $(\mathfrak{T}, u)$ be an object of $\operatorname{Conv}\left(X^{\prime} / \mathfrak{S}^{\prime}\right)$ and $g:(\mathfrak{Z}, w) \rightarrow \varphi(\mathfrak{T}, u)$ a morphism of $\operatorname{Conv}(X / \mathfrak{S})$. Then there exists an object $(\mathfrak{Z}, v)$ of $\operatorname{Conv}\left(X^{\prime} / \mathfrak{S}^{\prime}\right)$ and a morphism $h:(\mathfrak{Z}, v) \rightarrow(\mathfrak{T}, u)$ of $\operatorname{Conv}\left(X^{\prime} / \mathfrak{S}^{\prime}\right)$ such that $g=\varphi(h)$.

(ii) Equipped with the Zariski topology (resp. fppf topology) (4.3) on both sides, the functor $\varphi$ is continuous and cocontinuous.

Proof. (i) By considering compositions $\mathfrak{Z} \rightarrow \mathfrak{T} \rightarrow \mathfrak{S}^{\prime}$ and $Z_{0} \rightarrow T_{0} \rightarrow X^{\prime}$, we obtain an object $(\mathfrak{Z}, v)$ of $\operatorname{Conv}\left(X^{\prime} / \mathfrak{S}\right)$ and a morphism $h:(\mathfrak{Z}, v) \rightarrow(\mathfrak{T}, u)$ of $\operatorname{Conv}\left(X^{\prime} / \mathfrak{S}^{\prime}\right)$ such that $g=\varphi(h)$.

(ii) A family of morphisms $\left\{\left(\mathfrak{T}_{i}, u_{i}\right) \rightarrow(\mathfrak{T}, u)\right\}_{i \in I}$ of $\operatorname{Conv}\left(X^{\prime} / \mathfrak{S}^{\prime}\right)$ belongs to $\operatorname{Cov}_{\text {zar }}(\mathfrak{T}, u)$ (resp. $\left.\operatorname{Cov}_{\text {fppf }}(\mathfrak{T}, u)\right)$ if and only if, its image by $\varphi$ belongs to $\operatorname{Cov}_{\text {zar }}(\varphi(\mathfrak{T}, u))\left(\operatorname{resp} \cdot \operatorname{Cov}_{\text {fppf }}(\varphi(\mathfrak{T}, u))\right.$. Since $\varphi$ commutes with the fibered product, the continuity of $\varphi$ follows from ([3] III 1.6).

Let $\left\{\left(\mathfrak{T}_{i}, u_{i}\right) \rightarrow \varphi(\mathfrak{T}, u)\right\}_{i \in I}$ be an element of $\operatorname{Cov}_{\text {zar }}(\varphi(\mathfrak{T}, u))\left(\operatorname{resp} . \operatorname{Cov}_{\text {fppf }}(\varphi(\mathfrak{T}, u))\right)$. By (i), there exists an element $\left\{\left(\mathfrak{T}_{i}, u_{i}\right) \rightarrow(\mathfrak{T}, u)\right\}_{i \in I}$ of $\operatorname{Cov}_{\text {zar }}(\mathfrak{T}, u)\left(\operatorname{resp} \cdot \operatorname{Cov}_{\text {fppf }}(\mathfrak{T}, u)\right)$ mapping by $\varphi$ to the given element. Then, $\varphi$ is cocontinuous by ([3] III 2.1).

4.10. By 2.12 and 4.9, the functor $\varphi$ (4.8.2) induces morphisms of topoi

$$
f_{\text {conv }, \tau}:\left(X^{\prime} / \mathfrak{S}^{\prime}\right)_{\text {conv }, \tau} \rightarrow(X / \mathfrak{S})_{\operatorname{conv}, \tau},
$$

such that the pullback functor is induced by the composition with $\varphi$. For a sheaf $\mathscr{F}$ of $(X / \mathfrak{S})_{\operatorname{conv}, \tau}$ and an object $\mathfrak{T}$ of $\operatorname{Conv}\left(X^{\prime} / \mathfrak{S}^{\prime}\right)$, we have (4.6)

$$
\left(f_{\text {conv }, \tau}^{*}(\mathscr{F})\right)_{\mathfrak{T}}=\mathscr{F}_{\varphi(\mathfrak{T})} .
$$

For any morphism $g$ of $\operatorname{Conv}\left(X^{\prime} / \mathfrak{S}^{\prime}\right)$, the transition morphism of $f_{\text {conv }, \tau}^{*}(\mathscr{F})$ associated to $g$ (4.6) is equal to the transition morphism of $\mathscr{F}$ associated to $\varphi(g)$.

By considering inverse image functors, one verifies that the following diagram commutes (4.7.1)

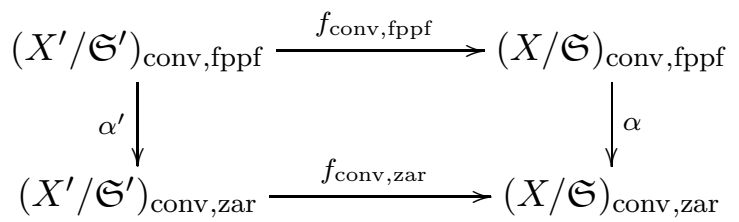

4.11. We define a presheaf of rings $\mathscr{O}_{X / \mathfrak{S}}\left[\frac{1}{p}\right]$ on $\operatorname{Conv}(X / \mathfrak{S})$ by

$$
(\mathfrak{T}, u) \mapsto \Gamma\left(\mathfrak{T}, \mathscr{O}_{\mathfrak{T}}\left[\frac{1}{p}\right]\right) .
$$

By fppf descent (2.8), $\mathscr{O}_{X / \mathfrak{S}}\left[\frac{1}{p}\right]$ is a sheaf for the fppf topology. Since the fppf topology is finer than the Zariski topology, it is also a sheaf for the Zariski topology.

For any object $(\mathfrak{T}, u)$ of $\operatorname{Conv}(X / \mathfrak{S})$, we have $\left(\mathscr{O}_{X / \mathfrak{S}}\left[\frac{1}{p}\right]\right)_{\mathfrak{T}}=\mathscr{O}_{\mathfrak{T}}\left[\frac{1}{p}\right]$. If $\mathscr{F}$ is an $\mathscr{O}_{X / \mathfrak{S}}\left[\frac{1}{p}\right]$-module of $(X / \mathfrak{S})_{\text {conv }, \tau}, \mathscr{F}_{\mathfrak{T}}$ is an $\mathscr{O}_{\mathfrak{T}}\left[\frac{1}{p}\right]$-module of $\mathfrak{T}_{\tau}$. For any morphism $f: \mathfrak{T}^{\prime} \rightarrow \mathfrak{T}$ of $\operatorname{Conv}(X / \mathfrak{S})$, the transition morphism $\gamma_{f}$ (4.6) extends to an $\mathscr{O}_{\mathfrak{T}^{\prime}}\left[\frac{1}{p}\right]$-linear morphism (2.9)

$$
c_{f}: f_{\tau}^{*}\left(\mathscr{F}_{\mathfrak{T}}\right) \rightarrow \mathscr{F}_{\mathfrak{T}^{\prime}}
$$

In view of [4.6, we deduce the following description for $\mathscr{O}_{X / \mathfrak{S}}\left[\frac{1}{p}\right]$-modules.

Proposition 4.12. For $\tau \in\{$ zar, fppf $\}$, an $\mathscr{O}_{X / \mathfrak{S}}\left[\frac{1}{p}\right]$-module of $(X / \mathfrak{S})_{\text {conv }, \tau}$ is equivalent to the following data:

(i) For every object $\mathfrak{T}$ of $\operatorname{Conv}(X / \mathfrak{S})$, an $\mathscr{O}_{\mathfrak{T}}\left[\frac{1}{p}\right]$-module $\mathscr{F}_{\mathfrak{T}}$ of $\mathfrak{T}_{\tau}$, 
(ii) For every morphism $f: \mathfrak{T}^{\prime} \rightarrow \mathfrak{T}$ of $\operatorname{Conv}(X / \mathfrak{S})$, an $\mathscr{O}_{\mathfrak{T}^{\prime}}$-linear morphism $c_{f}$ (4.11.2). which is subject to the following conditions

(a) If $f$ is the identity morphism, then $c_{f}$ is the identity.

(b) If $f: \mathfrak{T}^{\prime} \rightarrow \mathfrak{T}$ is a morphism of $f \mathbf{Z a r}_{/ \mathfrak{T}}$ (resp. $\mathbf{F f t} / \mathfrak{T}$ ), then $c_{f}$ is an isomorphism.

(c) If $f$ and $g$ are two composable morphisms, then we have $c_{g \circ f}=c_{f} \circ f_{\tau}^{*}\left(c_{g}\right)$.

Definition 4.13. Let $\mathscr{F}$ be an $\mathscr{O}_{X / \mathfrak{S}}\left[\frac{1}{p}\right]$-module of $(X / \mathfrak{S})_{\text {conv }, \tau}$.

(i) We say that $\mathscr{F}$ is coherent if for every object $\mathfrak{T}$ of $\operatorname{Conv}(X / \mathfrak{S}), \mathscr{F}_{\mathfrak{T}}$ is a coherent $(2.9)$.

(ii) We say that $\mathscr{F}$ is a crystal if for every morphism $f$ of $\operatorname{Conv}(X / \mathfrak{S}), c_{f}$ is an isomorphism.

With the notation of $4.10, f_{\text {conv }, \tau}^{*}$ sends coherent $\mathscr{O}_{X / \mathfrak{S}}\left[\frac{1}{p}\right]$-modules (resp. crystals) to coherent $\mathscr{O}_{X^{\prime} / \mathfrak{S}^{\prime}}\left[\frac{1}{p}\right]$-modules (resp. crystals).

4.14. Let $\mathscr{E}$ be a coherent crystal of $\mathscr{O}_{X / \mathfrak{S}}\left[\frac{1}{p}\right]$-modules of $(X / \mathfrak{S})_{\text {conv,zar. }}$ For any object $\mathfrak{T}$ of $\operatorname{Conv}(X / \mathfrak{S}), \alpha^{*}(\mathscr{E})_{\mathfrak{T}}$ (4.7.1) is the fppf sheaf associated to the presheaf

$$
\left(f: \mathfrak{T}^{\prime} \rightarrow \mathfrak{T}\right) \mapsto \Gamma\left(\mathfrak{T}^{\prime}, f_{\text {zar }}^{*}\left(\mathscr{E}_{\mathfrak{T}}\right)\right) .
$$

Since $\mathscr{E}$ is a coherent crystal, $\alpha^{*}(\mathscr{E})$ is equal to $\mathscr{E}$ as presheaves on $\operatorname{Conv}(X / \mathfrak{S})$ by fppf descent.

By 4.7, the direct image and inverse image functors of $\alpha$ induce an equivalence between the category of coherent crystals of $\mathscr{O}_{X / \mathfrak{S}}\left[\frac{1}{p}\right]$-modules of $(X / \mathfrak{S})_{\text {conv,zar }}$ and the category of coherent crystals of $\mathscr{O}_{X / \mathfrak{S}}\left[\frac{1}{p}\right]$-modules of $(X / \mathfrak{S})_{\text {conv,fppf }}$.

Following [26, 31], coherent crystal of $\mathscr{O}_{X / \mathfrak{S}}\left[\frac{1}{p}\right]$-modules of $(X / \mathfrak{S})_{\text {conv }, \tau}$ are called convergent isocrystals of $(X / \mathfrak{S})_{\text {conv }, \tau}$. We denote the full category of $\mathscr{O}_{X / \mathfrak{S}}\left[\frac{1}{p}\right]$-modules consisting of these objects by $\operatorname{Iso}^{\dagger}(X / \mathfrak{S})$.

We say that a convergent isocrystal $\mathscr{F}$ is locally projective if for every object $\mathfrak{T}$ of $\operatorname{Conv}(X / \mathfrak{S})$, $\mathscr{F}_{\mathfrak{T}}$ is locally projective of finite type $(\underline{2.3})$. In this case, $\mathscr{F}$ is locally projective $\mathscr{O}_{X / \mathfrak{S}^{\text {crys }} \text {-module of }}$ finite type.

Proposition 4.15 ([27] 0.7.2, [31] 2.35). Suppose that $X$ is smooth over $S$. There exists a canonical functor

$$
\iota: \operatorname{Iso}^{\dagger}(X / \mathfrak{S}) \rightarrow \mathscr{C}\left(\mathscr{O}_{X / \mathfrak{S}}^{\text {crys }}\right) \mathbb{Q}
$$

We briefly review the construction of the functor in the case where $X$ is separated and admits a smooth lifting $\mathfrak{X}$ over $\mathfrak{S}$. To do this, we need a formal $\mathfrak{X}$-groupoid constructed by admissible blow-up.

We set $\mathrm{Q}_{\mathfrak{X} / \mathfrak{S}}=\mathfrak{T}_{X, p}\left(\mathfrak{X}^{2}\right)$ the dilatation of the diagonal immersion $X \rightarrow \mathfrak{X}^{2}(2.10)$. By 2.11, the canonical morphism $\mathrm{Q}_{\mathfrak{X} / \mathfrak{S}, 1} \rightarrow X \times{ }_{S} X$ factors through the diagonal immersion. Then $\mathrm{Q}_{\mathfrak{X} / \mathfrak{S}}$ defines


with a formal $\mathfrak{X}$-groupoid structure (3.3) (cf. 34 4.12).

Let $\mathscr{E}$ be an object of $\operatorname{Iso}^{\dagger}(X / \mathfrak{S})$. The canonical morphisms $p_{1}, p_{2}: \mathrm{Q}_{\mathfrak{X} / \mathfrak{S}} \rightarrow \mathfrak{X}$ give rise to morphisms of $\operatorname{Conv}(X / \mathfrak{S})$. Since $\mathscr{E}$ is a crystal, $p_{1}, p_{2}$ induce isomorphisms

$$
p_{2}^{*}\left(\mathscr{E}_{\mathfrak{X}}\right) \underset{c_{p_{2}}}{\sim} \mathscr{E}_{\mathrm{Q}_{\mathfrak{X} / \mathfrak{S}}} \underset{c_{p_{1}}}{\stackrel{\sim}{\sim}} p_{1}^{*}\left(\mathscr{E}_{\mathfrak{X}}\right) \text {. }
$$

By a standard argument, one verifies that $\varepsilon=c_{p_{1}}^{-1} \circ c_{p_{2}}$ defines an $\mathscr{O}_{\mathrm{Q}_{\mathfrak{X} / \mathfrak{S}}}$-stratification on $\mathscr{E}_{\mathfrak{X}}$ (3.4).

Using 2.11, the canonical morphism $\mathrm{P}_{\mathfrak{X} / \mathfrak{S}} \rightarrow \mathfrak{X}^{2}$ induces a morphism of formal $\mathfrak{X}$-groupoids $\mathrm{P}_{\mathfrak{X} / \mathfrak{S}} \rightarrow \mathrm{Q}_{\mathfrak{X} / \mathfrak{S}}(\operatorname{cf}[34] 5.7)$. By taking inverse image, we obtain an $\mathscr{O}_{\mathrm{P}_{\mathfrak{X} / \mathfrak{S}}}$-strafitifcation on $\mathscr{E}_{\mathfrak{X}}$ and 


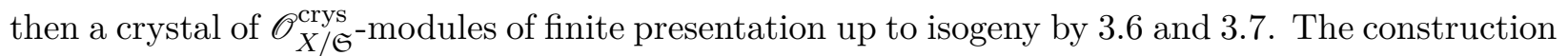
is clearly functorial.

4.16. There exists a canonical morphism of topoi $u_{X / \mathfrak{S}}:(X / \mathfrak{S})_{\text {conv,zar }} \rightarrow X_{\text {zar }}$ (cf. [27] §4). Suppose that $\mathfrak{S}$ is separated and that $X$ admits a smooth lifting $f: \mathfrak{X} \rightarrow \mathfrak{S}$. Let $\mathscr{E}$ be a convergent

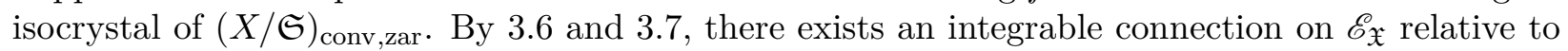
$\mathfrak{S}$ and we denote by $\mathscr{E}_{\mathfrak{X}} \otimes_{\mathscr{O}_{\mathfrak{X}}} \widehat{\Omega}_{\mathfrak{X} / \mathfrak{S}}^{\bullet}$ the associated de Rham complex. Then there exists a canonical isomorphism in the derived category $\mathrm{D}\left(\mathfrak{X}_{\text {zar }}, f^{-1}\left(\mathscr{O}_{\mathfrak{S}}\left[\frac{1}{p}\right]\right)\right)([31] 2.33)$

$$
\mathrm{R} u_{X / \mathfrak{S} *}(\mathscr{E}) \stackrel{\sim}{\rightarrow} \mathscr{E}_{\mathfrak{X}} \otimes_{\mathscr{O}_{\mathfrak{X}}} \widehat{\Omega}_{\mathfrak{X} / \mathfrak{S}}^{\bullet}
$$

Based on the above isomorphism, Shiho showed the following results.

Theorem 4.17 (31 2.36). Assume that $\mathfrak{S}$ is separated and that $X$ is smooth and proper over $S$. Let $\mathscr{E}$ be a convergent isocrystal of $(X / \mathfrak{S})_{\text {conv,zar }}$ and $g_{\text {conv,zar }}:(X / \mathfrak{S})_{\text {conv,zar }} \rightarrow(S / \mathfrak{S})_{\text {conv,zar }}$ the functorial morphism. Then there exists a canonical isomorphism in the derived category of $\mathscr{O}_{\mathfrak{S}}\left[\frac{1}{p}\right]$-modules (3.9, 4.15)

$$
\left(\mathrm{R} g_{\text {conv }, \operatorname{zar} *}(\mathscr{E})\right)_{\mathfrak{S}} \stackrel{\sim}{\rightarrow} \mathrm{R} g_{X / \mathfrak{S}, \operatorname{crys} *}(\iota(\mathscr{E})) .
$$

In particular, $\left(\mathrm{R}^{i} g_{\text {conv, zar } *(\mathscr{E}))_{\mathfrak{S}}}\right.$ is coherent for any $i \geq 0$.

Corollary 4.18 ([31] 2.37). Keep the assumption of 4.17 and suppose moreover that $\mathscr{E}$ is locally projective (4.14). Then, $\left(\mathrm{R} g_{\mathrm{conv}, \mathrm{zar} *}(\mathscr{E})\right)_{\mathfrak{S}}$ is a perfect complex of $\mathscr{O}_{\mathfrak{S}}\left[\frac{1}{p}\right]$-modules.

Corollary 4.19 ([31] 2.38). Let $\varphi: \mathfrak{S}^{\prime} \rightarrow \mathfrak{S}$ be an adic morphism of separated flat formal Wschemes of finite type, $X^{\prime}=X \times \times_{\mathfrak{S}} \mathfrak{S}^{\prime}$ and $\varphi_{\text {conv,zar }}:\left(X^{\prime} / \mathfrak{S}^{\prime}\right)_{\text {conv,zar }} \rightarrow(X / \mathfrak{S})_{\text {conv,zar }}$ the functorial morphism of convergent topoi (4.10.1). Then, for a locally projective convergent isocrystal $\mathscr{E}$ of



$$
\mathrm{L} \varphi_{\text {zar }}^{*}\left(\mathrm{R} g_{\text {conv,zar } *}(\mathscr{E})\right) \stackrel{\sim}{\rightarrow} \mathrm{R} g_{\text {conv,zar } *}^{\prime}\left(\varphi_{\text {conv,zar }}^{*}(\mathscr{E})\right) .
$$

\section{Higher DiRECT IMAGES OF A CONVERGENT ISOCRYSTAL ARE $p$-ADICALLY CONVERGENT}

5.1. In this section, we keep the notation of $\S$ 团and let $g: X \rightarrow Y$ denote a morphism of $S$-schemes.

Let $\mathfrak{T}$ be an object of $\operatorname{Conv}(Y / \mathfrak{S})$ and $\tau \in\{$ zar, fppf $\}$. By fppf descent for morphisms of formal W-schemes (1 5.12.1), the presheaf associated to $\mathfrak{T}$ is a sheaf for the fppf (resp. Zariski) topology that we denote by $\widetilde{\mathfrak{T}}$. We set $X_{T_{0}}=X \times_{Y} T_{0}$ and we denote by

$$
\begin{array}{cl}
g_{X / \mathfrak{T}, \tau}: & \left(X_{T_{0}} / \mathfrak{T}\right)_{\mathrm{conv}, \tau} \rightarrow\left(T_{0} / \mathfrak{T}\right)_{\mathrm{conv}, \tau}, \\
\omega_{\mathfrak{T}}: & \left(X_{T_{0}} / \mathfrak{T}\right)_{\mathrm{conv}, \tau} \rightarrow(X / \mathfrak{S})_{\mathrm{conv}, \tau}
\end{array}
$$

the functorial morphisms of topoi (4.10.1).

Lemma 5.2 ([4] V 3.2.2). There exists a canonical equivalence of topoi:

$$
(X / \mathfrak{S})_{\operatorname{conv}, \tau / g_{\text {conv }, \tau}^{*}(\widetilde{T})} \stackrel{\sim}{\rightarrow}\left(X_{T_{0}} / \mathfrak{T}\right)_{\operatorname{conv}, \tau}
$$

which identifies the localisation morphism and $\omega_{\mathfrak{T}}$.

The lemma can be verified in the same way as (4] V 3.2.2). 
Lemma 5.3 ([4] V 3.2.3). For any $\mathscr{O}_{X / \mathfrak{S}}\left[\frac{1}{p}\right]$-module $E$ of $(X / \mathfrak{S})_{\operatorname{conv}, \tau}$, there exists a canonical isomorphism in $\mathrm{D}^{+}\left(\mathfrak{T}_{\tau}, \mathscr{O}_{\mathfrak{T}}\left[\frac{1}{p}\right]\right)$

$$
\left(\mathrm{R} g_{\text {conv }, \tau *}(E)\right)_{\mathfrak{T}} \stackrel{\sim}{\rightarrow}\left(\mathrm{R} g_{X / \mathfrak{T}, \tau *}\left(\omega_{\mathfrak{T}}^{*}(E)\right)\right)_{\mathfrak{T}} .
$$

Proof. Let $E$ be an abelian sheaf of $(X / \mathfrak{S})_{\operatorname{conv}, \tau}$ and $f: \mathfrak{T}^{\prime} \rightarrow \mathfrak{T}$ a morphism of $\operatorname{Conv}(Y / \mathfrak{S})$. The morphism $f$ induces a functorial morphism of topoi $\varphi:\left(X_{T_{0}^{\prime}} / \mathfrak{T}^{\prime}\right)_{\text {conv }, \tau} \rightarrow\left(X_{T_{0}} / \mathfrak{T}\right)_{\text {conv }, \tau}$ which fits into the following commutative diagram

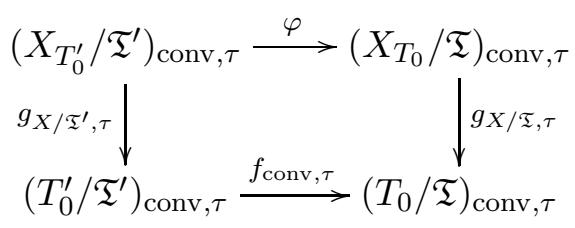

We have $\omega_{\mathfrak{T}^{\prime}}=\omega_{\mathfrak{T}} \circ \varphi$. By [5.2, $\varphi\left(\right.$ resp. $\left.f_{\text {conv }, \tau}\right)$ coincides with the localisation morphism on the sheaf $g_{X / \mathfrak{T}, \tau}^{*}\left(\widetilde{\mathfrak{T}}^{\prime}\right)$ (resp. $\left.\widetilde{\mathfrak{T}}^{\prime}\right)$. Then $g_{X / \mathfrak{T}, \tau *}\left(\omega_{\mathfrak{T}}^{*}(E)\right)$ is the sheaf associated to the presheaf on $\operatorname{Conv}\left(T_{0} / \mathfrak{T}\right)$

$$
\left(f: \mathfrak{T}^{\prime} \rightarrow \mathfrak{T}\right) \mapsto \Gamma\left(\left(X_{T_{0}^{\prime}} / \mathfrak{T}^{\prime}\right)_{\operatorname{conv}, \tau}, \omega_{\mathfrak{T}^{\prime}}^{*}(E)\right) .
$$

The sheaf $\left(g_{\operatorname{conv}, \tau *}(E)\right)_{\mathfrak{T}}$ is associated to the presheaf

$$
\left(f: \mathfrak{T}^{\prime} \rightarrow \mathfrak{T}\right) \mapsto \Gamma\left((X / \mathfrak{S})_{\operatorname{conv}, \tau / g_{\text {conv }, \tau}^{*}\left(\widetilde{\mathfrak{T}}^{\prime}\right)},\left.E\right|_{g_{\text {conv }, \tau}^{*}\left(\widetilde{\mathfrak{T}}^{\prime}\right)}\right) .
$$

By 5.2 , we deduce a canonical isomorphism of $\mathfrak{T}_{\tau}$

$$
\left(g_{\text {conv }, \tau *}(E)\right)_{\mathfrak{T}} \stackrel{\sim}{\rightarrow}\left(g_{X / \mathfrak{T}, \tau *}\left(\omega_{\mathfrak{T}}^{*}(E)\right)\right)_{\mathfrak{T}} .
$$

Since $\omega_{\mathfrak{T}}$ coincides with a localisation morphism, if $I^{\bullet}$ is an injective resolution of $E, \omega_{\mathfrak{T}}^{*}\left(I^{\bullet}\right)$ is an injective resolution of $\omega_{\mathfrak{T}}^{*}(E)$. Then the isomorphism (5.3.1) follows from (5.3.3).

Remark 5.4. Keep the notation of 5.3 . Let $f: \mathfrak{T}^{\prime} \rightarrow \mathfrak{T}$ be a morphism of $\operatorname{Conv}(Y / \mathfrak{S})$. It induces morphisms of topoi (5.3.2). We consider canonical morphisms

$$
f_{\tau}^{*}\left(\mathrm{R}^{i} g_{X / \mathfrak{T}, \tau *}\left(\omega_{\mathfrak{T}}^{*}(E)\right)_{\mathfrak{T}}\right) \rightarrow\left(f_{\text {conv }, \tau}^{*}\left(\mathrm{R}^{i} g_{X / \mathfrak{T}, \tau *}\left(\omega_{\mathfrak{T}}^{*}(E)\right)\right)_{\mathfrak{T}^{\prime}} \stackrel{\sim}{\rightarrow} \mathrm{R}^{i} g_{X / \mathfrak{T}^{\prime}, \tau *}\left(\omega_{\mathfrak{T}^{\prime}}^{*}(E)\right)_{\mathfrak{T}^{\prime}}\right.
$$

where the first morphism is the transition morphism of $\mathrm{R}^{i} g_{X / \mathfrak{T}, \tau *}\left(\omega_{\mathfrak{T}}^{*}(E)\right)$ associated to $f$ and the second one an isomorphism because $\omega_{\mathfrak{T}^{\prime}}=\omega_{\mathfrak{T}} \circ \varphi, \varphi, f_{\text {conv }, \tau}$ are localisation morphisms ([3] V 5.1).

In view of the proof of [5.3, via (5.3.1), the above composition is compatible with the transition morphism of $\mathrm{R}^{i} g_{\text {conv }, \tau *}(E)$ associated to $f$

$$
f_{\tau}^{*}\left(\left(\mathrm{R}^{i} g_{\mathrm{conv}, \tau *}(E)\right)_{\mathfrak{T}}\right) \rightarrow\left(\mathrm{R}^{i} g_{\mathrm{conv}, \tau *}(E)\right)_{\mathfrak{T}^{\prime}} .
$$

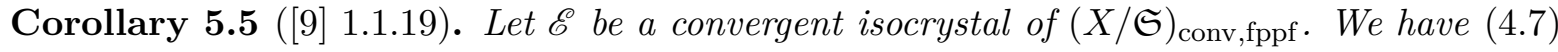

$$
\mathrm{R}^{i} \alpha_{*}(\mathscr{E})=0, \quad \forall i \geq 1 .
$$

The assertion can be verified in the same way as ([9] 1.1.19).

Corollary 5.6. Let $\mathfrak{S}^{\prime} \rightarrow \mathfrak{S}$ be a morphism of $\mathbf{S}^{\diamond}$ (2.1), $Y^{\prime}$ an $S^{\prime}$-scheme and $h: Y^{\prime} \rightarrow Y a$ morphism compatible with $S^{\prime} \rightarrow S$. We set $X^{\prime}=X \times_{Y} Y^{\prime}$ and we denote by $g^{\prime}: X^{\prime} \rightarrow Y^{\prime}$ and 
$h^{\prime}: X^{\prime} \rightarrow X$ the canonical morphisms:



Then, for any $\mathscr{O}_{X / \mathfrak{S}}\left[\frac{1}{p}\right]$-module $E$ of $(X / \mathfrak{S})_{\text {conv }, \tau}$, the base change morphism

$$
h_{\mathrm{conv}, \tau}^{*}\left(\mathrm{R} g_{\mathrm{conv}, \tau *}(E)\right) \stackrel{\sim}{\rightarrow} \mathrm{R} g_{\mathrm{conv}, \tau *}^{\prime}\left(h_{\mathrm{conv}, \tau}^{\prime *}(E)\right),
$$

is an isomorphism.

Proof. Let $\mathfrak{T}$ be an object of $\operatorname{Conv}\left(Y^{\prime} / \mathfrak{S}^{\prime}\right)$. We denotes abusively the image of $\mathfrak{T}$ in $\operatorname{Conv}(Y / \mathfrak{S})$ by $\mathfrak{T}$. We set $X_{T_{0}}=T_{0} \times_{Y} X\left(=T_{0} \times_{Y^{\prime}} X^{\prime}\right)$. By applying 5.3 to $g$ and $g^{\prime}$, one verifies that the valuation of two sides of (5.6.1) at $\mathfrak{T}$ are both isomorphic to $\left(\mathrm{R} g_{X / \mathfrak{T}, \tau *}\left(\omega_{\mathfrak{T}}^{*}(E)\right)\right)_{\mathfrak{T}}$ and that (5.6.1) induces an isomorphism between them. Then the assertion follows.

5.7. In the following, we consider the case where $\mathfrak{S}=\operatorname{Spf}(\mathrm{W})$ and $X, Y$ are schemes over $S=$ $\operatorname{Spec}(k)$. We denote by $\operatorname{pConv}(X / \mathrm{W})$ the full subcategory of $\operatorname{Conv}(X / \mathrm{W})$ consisting of objects $(\mathfrak{T}, u)$ such that $u$ can be lifted to a $k$-morphism $\tilde{u}: T \rightarrow X$. Given an object $\mathfrak{T}$ of $\operatorname{pConv}(X / \mathrm{W})$ and a morphism $f: \mathfrak{T}^{\prime} \rightarrow \mathfrak{T}$ of $\operatorname{Conv}(X / \mathrm{W})$, then $\mathfrak{T}^{\prime}$ is still an object of $\mathrm{pConv}(X / \mathrm{W})$. Objects of $p \operatorname{Conv}(X / \mathrm{W})$ are called " $p$-adic enlargements" in [26].

Lemma 5.8. Suppose that $X$ is smooth over $k$. A convergent isocrystal $\mathscr{E}$ of $(X / \mathrm{W})_{\mathrm{conv}, \mathrm{zar}}$ is locally projective (4.14).

Proof. The question being local, we may assume that $X$ admits a smooth lifting $\mathfrak{X}$ over W. By 3.8 and 4.15, $\mathscr{E}_{\mathfrak{X}}$ is locally projective. Since every object $\mathfrak{T}$ of $\operatorname{Conv}(X / \mathrm{W})$ locally admits a morphism to $\mathfrak{X}$, we deduce that $\mathscr{E}$ is locally projective.

Proposition 5.9. Suppose that $Y$ is smooth over $k$ and admits a smooth lifting $\mathfrak{Y}$ over $\mathrm{W}$ and that $g: X \rightarrow Y$ is smooth and proper. Let $\mathscr{E}$ be a convergent isocrystal of $(X / \mathrm{W})_{\text {conv,zar. Then there }}$ exists an $\mathscr{O}_{\mathrm{P}_{\mathfrak{Y} / \mathfrak{S}}}$-stratification on $\left(\mathrm{R}^{i} g_{\mathrm{conv}, \mathrm{zar} *}(\mathscr{E})\right)_{\mathfrak{Y}}$. In particular the later is locally projective of finite type (2.3).

Proof. We take again the notation of 4.15 for $\mathfrak{Y} \rightarrow \operatorname{Spf}(\mathrm{W})$ and we set $\mathfrak{Z}=\mathrm{Q}_{\mathfrak{Y} / \mathrm{W}}$ that we consider as an object of $\operatorname{Conv}(Y / \mathrm{W})$, and $\mathscr{F}=\mathrm{R}^{i} g_{\operatorname{conv}, \text { zar } *}(\mathscr{E})$. By 5.3, we have canonical isomorphisms

$$
\mathscr{F}_{\mathfrak{Y}} \stackrel{\sim}{\rightarrow}\left(\mathrm{R}^{i} g_{X / \mathfrak{Y}, \text { zar } *}\left(\omega_{\mathfrak{Y}}^{*}(\mathscr{E})\right)\right)_{\mathfrak{Y}}, \quad \mathscr{F}_{\mathfrak{Z}} \stackrel{\sim}{\rightarrow}\left(\mathrm{R}^{i} g_{X / \mathfrak{Z}, \text { zar } *}\left(\omega_{\mathfrak{Z}}^{*}(\mathscr{E})\right)\right)_{\mathfrak{Z}}
$$

By 4.17, $\mathscr{F}_{\mathfrak{Y}}$ is coherent. The projections $p_{1}, p_{2}: \mathfrak{Z} \rightarrow \mathfrak{Y}$ define two morphisms of $\operatorname{Conv}(Y / \mathrm{W})$ and induce two morphisms of topoi

$$
\begin{aligned}
\left(X_{Z_{0}} / \mathfrak{Z}\right)_{\text {conv,zar }} \stackrel{\sim}{\rightarrow}\left(X \times_{Y, p_{1}} Z / \mathfrak{Z}\right)_{\text {conv,zar }} \rightarrow(X / \mathfrak{Y})_{\text {conv,zar }} \\
\left(X_{Z_{0}} / \mathfrak{Z}\right)_{\text {conv,zar }} \stackrel{\sim}{\rightarrow}\left(X \times_{Y, p_{2}} Z / \mathfrak{Z}\right)_{\text {conv,zar }} \rightarrow(X / \mathfrak{Y})_{\text {conv,zar }} .
\end{aligned}
$$

Since $X$ is smooth over $k, \mathscr{E}$ is locally projective by [5.8. The projections $p_{1}, p_{2}$ are rig-flat ([1] 5.4.12). By 4.19 and 5.4, $p_{1}, p_{2}$ induce isomorphisms

$$
p_{2}^{*}\left(\mathscr{F}_{\mathfrak{Y}}\right) \underset{c_{p_{2}}}{\sim} \mathscr{F}_{\mathfrak{Z}} \underset{c_{p_{1}}}{\stackrel{\sim}{\sim}} p_{1}^{*}\left(\mathscr{F}_{\mathfrak{Y}}\right)
$$


By a standard argument, the isomorphism $c_{p_{1}}^{-1} \circ c_{p_{2}}$ defines an $\mathscr{O}_{\mathrm{Q}_{\mathfrak{Y} / \mathrm{w}}}$-stratification on $\mathscr{F}_{\mathfrak{Y}}$. Taking pull-back by $\mathrm{P}_{\mathfrak{Y} / \mathrm{W}} \rightarrow \mathrm{Q}_{\mathfrak{Y} / \mathrm{W}}$ (4.15), we obtain an an $\mathscr{O}_{\mathrm{P}_{\mathfrak{Y} / \mathrm{W}}}$-stratification on $\mathscr{F}_{\mathfrak{Y}}$. The second assertion follows from 3.8 .

Proposition 5.10. Suppose that $Y$ is smooth over $k$ and that $g: X \rightarrow Y$ is smooth and proper. Let $\mathscr{E}$ be a convergent isocrystal of $(X / \mathrm{W})_{\mathrm{conv}, \tau}$ for $\tau \in\{\mathrm{zar}, \mathrm{fppf}\}$ and $i$ an integer $\geq 0$. We have:

(i) For every object $\mathfrak{T}$ of $\mathrm{pConv}(Y / \mathrm{W})$ (5.7), $\mathrm{R}^{i} g_{\mathrm{conv}, \tau *}(\mathscr{E})_{\mathfrak{T}}$ is coherent (2.9).

(ii) For every morphism $f$ of $\mathrm{pConv}(Y / \mathrm{W})$, the associated transition morphism $c_{f}$ of $\mathrm{R}^{i} g_{\mathrm{conv}, \tau *}(\mathscr{E})$ is an isomorphism.

By [5.8, $\mathscr{E}$ is locally projective. We set $\mathscr{F}_{\tau}^{i}=\mathrm{R}^{i} g_{\text {conv }, \tau *}(\mathscr{E})$ and $\mathscr{G}_{\tau}^{i}=\left(\mathrm{R}^{i} g_{X / \mathfrak{T}, \tau *}\left(\omega_{\mathfrak{T}}^{*}(\mathscr{E})\right)\right)$ (5.1). By [5.3, we have a canonical isomorphism

$$
\mathscr{F}_{\tau, \mathfrak{T}}^{i} \stackrel{\sim}{\rightarrow} \mathscr{G}_{\tau, \mathfrak{T}}^{i} .
$$

5.11. Proof of 5.10 for Zariski topology.

(i) Since $(\mathfrak{T}, u)$ is an object of $\operatorname{pConv}(Y / \mathrm{W})$, we take a lifting $\widetilde{u}: T \rightarrow Y$ of $u$ and we set $X_{T}=X \times_{Y} T$. Then, we have a canonical equivalence $\left(X_{T_{0}} / \mathfrak{T}\right)_{\text {conv }, \tau} \stackrel{\sim}{\rightarrow}\left(X_{T} / \mathfrak{T}\right)_{\text {conv }, \tau}$ (4.1) and the assertion follows from 4.17 .

(ii) The question being local, by [5.6, we may therefore assume that $Y$ is affien and admits a smooth lifting $\mathfrak{Y}$ over $\mathrm{W}$. Then $\mathscr{F}_{\text {zar }, \mathfrak{Y}}^{i}$ and $\mathscr{G}_{\text {zar, } \mathfrak{Y}}^{i}$ are locally projective of finite type by [5.9.

We first prove assertion (ii) for a morphism $h: \mathfrak{T} \rightarrow \mathfrak{Y}$ of $\mathrm{pConv}(Y / \mathrm{W})$ with target $\mathfrak{Y}$. By 4.19, we have a spectral sequence:

$$
\mathrm{E}_{2}^{i-j, j}=\mathrm{L}_{i-j} h_{\text {zar }}^{*}\left(\mathscr{G}_{\text {zar }, \mathfrak{Y}}^{j}\right) \Rightarrow \mathscr{G}_{\text {zar }, \mathfrak{T}}^{i} .
$$

Since each $\mathscr{G}_{\text {zar, } \mathfrak{Y}}^{j}$ is locally projective of finite type, we deduce that $\mathrm{E}_{2}^{i-j, j}=0$ for $i \neq j$. Then the

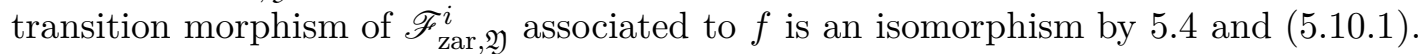

Since the question is local, for a general morphism $f:\left(\mathfrak{T}^{\prime}, u^{\prime}\right) \rightarrow(\mathfrak{T}, u)$ of $\operatorname{pConv}(Y / \mathrm{W})$, we may assume that $u$ can be lifted to a morphism $h: \mathfrak{T} \rightarrow \mathfrak{Y}$ of $\operatorname{pConv}(Y / \mathrm{W})$. By the previous result, $c_{h}$ and $c_{h \circ f}$ are isomorphisms. Then we deduce that $c_{f}$ is an isomorphism by 4.12 (c).

5.12. Proof of 5.10 for fppf topology. We consider the presheaf $\mathscr{P}$ on $\operatorname{Conv}(Y / \mathrm{W})$ defined by

$$
(\mathfrak{T}, u) \mapsto \mathrm{H}^{i}\left(\left(X_{T_{0}} / \mathfrak{T}\right)_{\text {conv,fppf }}, \omega_{\mathfrak{T}}^{*}(\mathscr{E})\right) .
$$

By [5.5, the right hand side is isomorphic to $\mathrm{H}^{i}\left(\left(X_{T_{0}} / \mathfrak{T}\right)_{\text {conv,zar }}, \omega_{\mathfrak{T}}^{*}\left(\alpha_{*}(\mathscr{E})\right)\right)$. We set $\mathscr{F}_{\text {zar }}^{i}=$ $\mathrm{R}^{i} g_{\text {conv,zar } *}\left(\alpha_{*}(\mathscr{E})\right)$. By [5.3, the fppf (resp. Zariski) sheaf associated to $\mathscr{P}$ is $\mathscr{F}_{\text {fppf }}^{i}$ (resp. $\mathscr{F}_{\text {zar }}^{i}$ ). Then we deduce a canonical isomorphism (4.7.1)

$$
\alpha^{*}\left(\mathscr{F}_{\text {zar }}^{i}\right) \stackrel{\sim}{\rightarrow} \mathscr{F}_{\text {fppf }}^{i} .
$$

Let $\mathfrak{T}$ be an object of $\operatorname{pConv}(Y / \mathrm{W})$. By 5.10 for Zariski topology and fppf descent, we deduce that $\mathscr{F}_{\text {fppf }, \mathfrak{T}}^{i}$ is the fppf sheaf associated to the coherent $\mathscr{O}_{\mathfrak{T}}\left[\frac{1}{p}\right]$-module $\mathscr{F}_{\text {zar, } \mathfrak{T}}^{i}(2.9)$ and hence is coherent. Assertion (i) follows.

Since $\mathscr{F}_{\text {fppf, } \mathfrak{T}}^{i}$ is the fppf sheaf associated to $\mathscr{F}_{\text {zar, }}^{i}$, assertion (ii) follows from 5.10 (ii) for Zariski topology and (5.12.1). 


\section{Frobenius Descents}

6.1. In this section, $\mathfrak{S}$ denotes a flat formal $\mathrm{W}$-scheme of finite type. Suppose that the Frobenius morphism $F_{S_{0}}: S_{0} \rightarrow S_{0}$ of the reduced subscheme of $S$ is flat (and hence faithfully flat).

Let $X$ be an $S_{0}$-scheme locally of finite type. We denote by $X^{\prime}$ the base change of $X$ by $F_{S_{0}}$ and by $F_{X / S_{0}}: X \rightarrow X^{\prime}$ the relative Frobenius morphism of $X$ relative to $S_{0}$. Then we have a commutative diagram

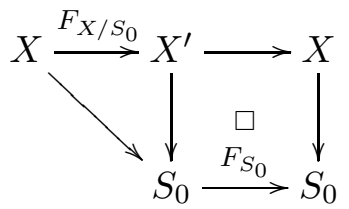

We study the functorial morphism of convergent topoi induced by the relative Frobenius morphism $F_{X / S_{0}}: X \rightarrow X^{\prime}$. We denote the functor (4.8.2) induced by $F_{X / S_{0}}$ by:

$$
\begin{aligned}
\rho: \operatorname{Conv}(X / \mathfrak{S}) & \rightarrow \operatorname{Conv}\left(X^{\prime} / \mathfrak{S}\right), \\
(\mathfrak{T}, u) & \mapsto\left(\mathfrak{T}, F_{X / S_{0}} \circ u\right) .
\end{aligned}
$$

Note that $F_{X / S_{0}} \circ u=u^{\prime} \circ F_{T_{0} / S_{0}}$.

Lemma 6.2. Let $Y$ be a reduced $S_{0}$-scheme, $Z$ an $S_{0}$-scheme and $g_{1}, g_{2}: Y \rightarrow Z$ two $S_{0}$-morphisms. We put $h_{i}=g_{i}^{\prime} \circ F_{Y / S_{0}}: Y \rightarrow Y^{\prime} \rightarrow Z^{\prime}$ for $i=1,2$. If $h_{1}=h_{2}$, then $g_{1}=g_{2}$.

Proof. Since $F_{Y / S_{0}}$ is a homeomorphism and $h_{1}=h_{2}$, then $\left|g_{1}\right|=\left|g_{2}\right|$ on the underlying topological spaces. Since the question is local, we can reduce to the case where $Y, Z, S_{0}$ are affine.

Since $Y$ is reduced and separated over $S_{0}, F_{Y / S_{0}}$ is schematically dominant ([22] 5.4.2) and we deduce that $g_{1}^{\prime}=g_{2}^{\prime}$ (22] 5.4.1). The Frobenius morphism $F_{S_{0}}$ is faithfully flat. Then the functor $Y \mapsto Y^{\prime}$ from the category of affine $S_{0}$-schemes to itself is faithful. The lemma follows.

Lemma 6.3. The functor $\rho$ is fully faithful.

Proof. The functor $\rho$ is clearly faithful. We prove its fullness. Let $\left(\mathfrak{T}_{1}, u_{1}\right),\left(\mathfrak{T}_{2}, u_{2}\right)$ be two objects of $\operatorname{Conv}(X / \mathfrak{S})$ and $g: \rho\left(\mathfrak{T}_{1}, u_{1}\right) \rightarrow \rho\left(\mathfrak{T}_{2}, u_{2}\right)$ a morphism of $\operatorname{Conv}\left(X^{\prime} / \mathfrak{S}\right)$. We set $g_{0}: T_{1,0} \rightarrow T_{2,0}$ the induced morphism. To show that the morphism $\mathfrak{T}_{1} \rightarrow \mathfrak{T}_{2}$ define a morphism of $\operatorname{Conv}(X / \mathfrak{S})$ which is sent to $g$ by $\rho$, it suffices to show that $u_{1}=u_{2} \circ g_{0}$. Since $g$ is a morphism of $\operatorname{pConv}\left(X^{\prime} / \mathfrak{S}\right)$, we have a commutative diagram

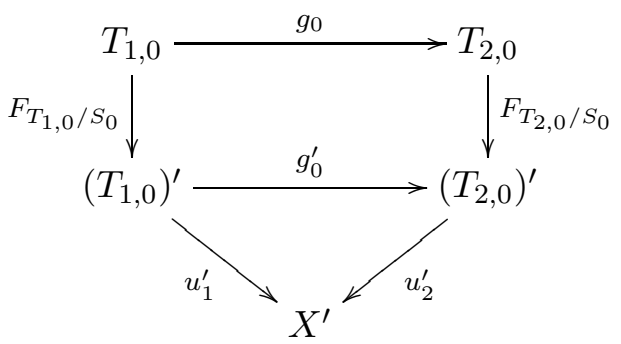

Then the assertion follow from 6.2 applied to $u_{1}$ and $u_{2} \circ g_{0}$.

Lemma 6.4. (i) Let $(\mathfrak{T}, u)$ be an object of $\operatorname{Conv}\left(X^{\prime} / \mathfrak{S}\right)$ such that $\mathfrak{T}$ is affine and that $u: T_{0} \rightarrow X^{\prime}$ factor through an affine open subscheme $U^{\prime}$ of $X^{\prime}$. Then there exists an object $(\mathfrak{Z}, v)$ of $\operatorname{Conv}(X / \mathfrak{S})$ and a fppf covering $\{f: \rho(\mathfrak{Z}, v) \rightarrow(\mathfrak{T}, u)\}$ in $\operatorname{Conv}\left(X^{\prime} / \mathfrak{S}\right)$. 
(ii) Keep the assumption and notation of (i). Let $g:\left(\mathfrak{T}_{1}, u_{1}\right) \rightarrow(\mathfrak{T}, u)$ be a morphism of $\operatorname{Conv}\left(X^{\prime} / \mathfrak{S}\right)$. Then there exists a morphism $h:\left(\mathfrak{Z}_{1}, v_{1}\right) \rightarrow(\mathfrak{Z}, v)$ of $\operatorname{Conv}(X / \mathfrak{S})$ and a fppf covering $\left\{\varphi: \rho\left(\mathfrak{Z}_{1}, v_{1}\right) \rightarrow\left(\mathfrak{T}_{1}, u_{1}\right)\right\}$ such that the following diagram is Cartesian:

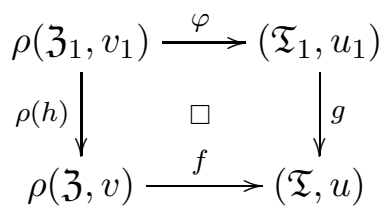

(iii) Every object of $\operatorname{Conv}\left(X^{\prime} / \mathfrak{S}\right)$ admits a Zariski covering whose objects satisfying conditions of (i).

Proof. (i) We set $U=F_{X / S_{0}}^{-1}\left(U^{\prime}\right)$ which is an affine $S_{0}$-scheme of finite type and we take a closed $S_{0}$-immersion $\iota_{0}: U \rightarrow Y_{0}=\operatorname{Spec}\left(\mathscr{O}_{S_{0}}\left[T_{1}, \cdots, T_{d}\right]\right)$. We denote by $\mathfrak{Y}=\operatorname{Spf}\left(\mathscr{O}_{\mathfrak{S}}\left\{T_{1}, \cdots, T_{d}\right\}\right)$ and $F: \mathfrak{Y} \rightarrow \mathfrak{Y}$ the $\mathfrak{S}$-morphism defined by sending each $T_{i}$ to $T_{i}^{p}$.

Note that $Y_{0}^{\prime}=Y_{0}$ and the restriction of $F$ on $Y_{0}$ is same the relative Frobenius morphism $F_{Y_{0} / S_{0}}$. We have a commutative diagram



and a canonical morphism $U \rightarrow U^{\prime} \times_{Y_{0}, F} Y_{0}$. We denote the composition of $\iota_{0}^{\prime}: U^{\prime} \rightarrow Y_{0}$ and $Y_{0} \rightarrow \mathfrak{Y}$ by $\iota^{\prime}$. Since $\mathfrak{Y}$ is smooth over $\mathfrak{S}$, there exists an $\mathfrak{S}$-morphism $\tau: \mathfrak{T} \rightarrow \mathfrak{Y}$ lifting $\iota^{\prime} \circ u: T_{0} \rightarrow \mathfrak{Y}$. We consider the following commutative diagram:

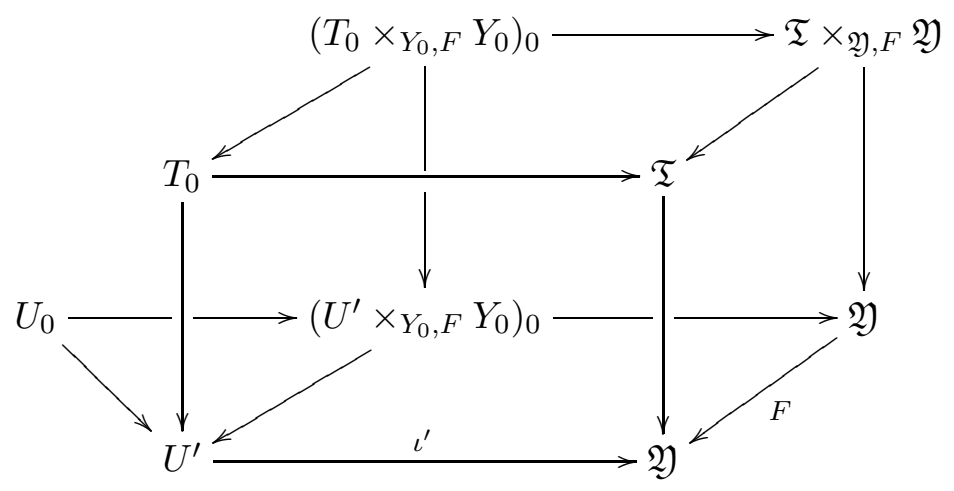

where $U_{0} \rightarrow U^{\prime}$ is induced by $F_{U / S_{0}}$.

If the ideal sheaf associated to $\iota_{0}: U \hookrightarrow Y_{0}$ is locally generated by polynomials $\left\{f_{1}, \cdots, f_{n}\right\}$ of $\mathscr{O}_{S_{0}}\left[T_{1}, \cdots, T_{d}\right]$, the ideal sheaf associated to $\iota_{0}^{\prime} \times_{Y_{0}, F} Y_{0}: U^{\prime} \times_{Y_{0}, F} Y_{0} \hookrightarrow Y_{0}$ (6.4.2) is locally generated by $\left\{f_{1}^{p}, \cdots, f_{n}^{p}\right\}$. Then the canonical morphism $U \rightarrow U^{\prime} \times_{Y_{0}, F} Y_{0}$ induces an isomorphism

$$
U_{0} \stackrel{\sim}{\rightarrow}\left(U^{\prime} \times_{Y_{0}, F} Y_{0}\right)_{0} .
$$

By (6.4.3) and $(22$ 4.5.11), we obtain an object $(\mathfrak{T} \times \mathfrak{Y}, F \mathfrak{Y}, v)$ of $\operatorname{Conv}(X / \mathfrak{S})$ and a morphism $f: \rho(\mathfrak{T} \times \mathfrak{Y}, F \mathfrak{Y}, v) \rightarrow(\mathfrak{T}, u)$ of $\operatorname{Conv}\left(X^{\prime} / \mathfrak{S}\right)$. Since the reduction modulo $p$ of $F$ is faithfully flat of finite type ([19] 3.2), so is $F$ (cf. [34] 7.2). Then, $f$ is a fppf covering (4.3). 
(ii) We denote by $\left(\mathfrak{Z}_{1}, w\right)$ the fibered product $\rho(\mathfrak{Z}, v) \times_{(\mathfrak{T}, u)}\left(\mathfrak{T}_{1}, u_{1}\right)$ in $\operatorname{Conv}\left(X^{\prime} / \mathfrak{S}\right)$. By applying 4.9 to the projection $\left(\mathfrak{Z}_{1}, w\right) \rightarrow \rho(\mathfrak{Z}, v)$, we obtain the Cartesian diagram (6.4.1). Since $\varphi$ is the base change of $f, \varphi$ is a fppf covering.

(iii) Let $(\mathfrak{T}, u)$ be an object of $\operatorname{Conv}\left(X^{\prime} / \mathfrak{S}\right)$ and $U^{\prime}$ an affine open subscheme of $X^{\prime}$. We denote by $\mathfrak{T}_{U^{\prime}}$ the open formal subscheme of $\mathfrak{T}$ associated to the open subset $u^{-1}\left(\left|U^{\prime}\right|\right)$ of $\left|T_{0}\right|=|T|$. The assertion follows by taking an affine covering of $\mathfrak{T}_{U^{\prime}}$ for every $U^{\prime}$.

Lemma 6.5. Let $\mathfrak{T}$ an object of $\operatorname{Conv}\left(X^{\prime} / \mathfrak{S}\right), \mathfrak{Z}$ an object of $\operatorname{Conv}(X / \mathfrak{S})$ and $\{\rho(\mathfrak{Z}) \rightarrow \mathfrak{T}\}$ a morphism of $\operatorname{Conv}\left(X^{\prime} / \mathfrak{S}\right)$. Then there exists an object $\mathfrak{Z} \times_{\mathfrak{T}} \mathfrak{Z}$ of $\operatorname{Conv}(X / \mathfrak{S})$ and two morphisms $p_{1}, p_{2}: \mathfrak{Z} \times \mathfrak{T} \mathfrak{Z} \rightarrow \mathfrak{Z}$ of $\operatorname{Conv}(X / \mathfrak{S})$ such that $\rho(\mathfrak{Z} \times \mathfrak{T} \mathfrak{Z})=\rho(\mathfrak{Z}) \times \mathfrak{T} \rho(\mathfrak{Z})$ and that $\rho\left(p_{1}\right)$ (resp. $\rho\left(p_{2}\right)$ ) is the projection $\rho(\mathfrak{Z}) \times \mathfrak{T} \rho(\mathfrak{Z}) \rightarrow \rho(\mathfrak{Z})$ on the first (resp. second) component.

Proof. By applying 4.9 (i) to the projection $\rho(\mathfrak{Z}) \times \mathfrak{T} \rho(\mathfrak{Z}) \rightarrow \rho(\mathfrak{Z})$ on the first component, we obtain an object $\mathfrak{Z} \times \mathfrak{T} \mathfrak{Z}$ of $\operatorname{Conv}(X / \mathfrak{S})$ and a morphism $p_{1}: \mathfrak{Z} \times \mathfrak{T} \mathfrak{Z} \rightarrow \mathfrak{Z}$ as in the proposition. The existence of $p_{2}$ follows from the fullness of $\rho$ (6.3).

Theorem 6.6. Suppose that the Frobenius morphism $F_{S_{0}}: S_{0} \rightarrow S_{0}$ flat. For every $S_{0}$-scheme locally of finite type $X$, the relative Frobenius morphism $F_{X / S_{0}}$ induces an equivalence of topoi.

$$
F_{X / S_{0}, \text { conv,fppf }}:(X / \mathfrak{S})_{\text {conv,fppf }} \stackrel{\sim}{\rightarrow}\left(X^{\prime} / \mathfrak{S}\right)_{\text {conv,fppf }} .
$$

Proof. The theorem follows from 2.13, 4.9, 6.3 and 6.4,

Proposition 6.7. Keep the assumption of 6.6. The inverse image and the direct image functors of $F_{X / S_{0}, \mathrm{conv}, \mathrm{zar}}$ induce equivalences of categories quasi-inverse to each other (4.13)

$$
\operatorname{Iso}^{\dagger}(X / \mathfrak{S}) \rightleftarrows \operatorname{Iso}^{\dagger}\left(X^{\prime} / \mathfrak{S}\right) \text {. }
$$

Proof. By 4.14, convergent isocrystals are sheaves for fppf topology and we work with fppf topology in this proof. We write simply (6.6.1) for $F_{X / S_{0}}$ and we will show that the direct image and inverse image functors of $F_{X / S_{0}}$ send coherent crystals of $\mathscr{O}_{X / \mathfrak{S}}\left[\frac{1}{p}\right]$-modules to coherent crystals of $\mathscr{O}_{X^{\prime} / \mathfrak{S}}\left[\frac{1}{p}\right]$ modules. The assertion for inverse image follows from (4.10.2) and we will prove it for direct image.

Let $\mathscr{F}$ be coherent crystal of $\mathscr{O}_{X / \mathfrak{S}}\left[\frac{1}{p}\right]$-modules and $(\mathfrak{T}, u)$ an object of $\operatorname{Conv}\left(X^{\prime} / \mathfrak{S}\right)$. We first show that $\left(F_{X / S_{0} *}(\mathscr{F})\right)_{\mathfrak{T}}$ is coherent. By $6.4($ iii), we may assume that $(\mathfrak{T}, u)$ satisifies conditions of 6.4(i). Then, by 6.4(ii) and 6.5, there exist objects $\mathfrak{Z}$ and $\mathfrak{Z} \times \mathfrak{T} \mathfrak{Z}$ of $\operatorname{Conv}(X / \mathfrak{S})$, a fppf covering $\{f: \rho(\mathfrak{Z}) \rightarrow \mathfrak{T}\}$ and two morphisms $p_{1}, p_{2}: \mathfrak{Z} \times \mathfrak{T} \mathfrak{Z} \rightarrow \mathfrak{Z}$ such that $\rho(\mathfrak{Z} \times \mathfrak{T} \mathfrak{Z})=\rho(\mathfrak{Z}) \times \mathfrak{T} \rho(\mathfrak{Z})$ and that $\rho\left(p_{1}\right)$ and $\rho\left(p_{2}\right)$ are the canonical projections of $\rho(\mathfrak{Z}) \times_{\mathfrak{T}} \rho(\mathfrak{Z})$. In particular, the morphism of formal schemes $\mathfrak{Z} \times \mathfrak{T} \mathfrak{Z} \rightarrow \mathfrak{Z}$ attached to $p_{1}$ (resp. $p_{2}$ ) is the projection on the first (resp. second) component.

Since the adjunction morphism $F_{X / S_{0}}^{*} F_{X / S_{0} *} \rightarrow$ id is an isomorphism (6.6), we have (4.10.2)

$$
\left(F_{X / S_{0} *}(\mathscr{F})\right)_{\rho(\mathfrak{Z})}=\mathscr{F}_{\mathfrak{Z}}, \quad\left(F_{X / S_{0} *}(\mathscr{F})\right)_{\rho(\mathfrak{Z} \times \mathfrak{I} \mathfrak{Z})}=\mathscr{F}_{\mathfrak{Z} \times \mathfrak{I} \mathfrak{Z} .}
$$

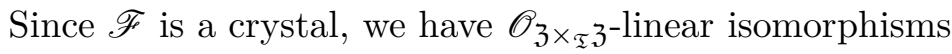

$$
p_{2}^{*}\left(\mathscr{F}_{\mathfrak{Z}}\right) \stackrel{c_{p_{2}}}{\sim} \mathscr{F}_{\mathfrak{Z} \times \mathfrak{T} \mathfrak{Z}} \stackrel{c_{p_{1}}}{\sim} p_{1}^{*}\left(\mathscr{F}_{\mathfrak{Z}}\right) .
$$

Then we obtain a descent datum $\left(\mathscr{F}_{\mathfrak{Z}}, c_{p_{1}}^{-1} \circ c_{p_{2}}\right)$ for the fppf covering $\{f: \mathfrak{Z} \rightarrow \mathfrak{T}\}$. By 2.8, there exists a coherent $\mathscr{O}_{\mathfrak{T}}\left[\frac{1}{p}\right]$-module $\mathscr{M}$ and a canonical $\mathscr{O}_{\mathfrak{Z}}$-linear isomorphism $f^{*}(\mathscr{M}) \stackrel{\sim}{\rightarrow} \mathscr{F}_{\mathfrak{Z}}$.

On the other hand, since $F_{X / S_{0} *}(\mathscr{F})$ is a sheaf fppf topology, there exists an exact sequence

$$
0 \rightarrow\left(F_{X / S_{0} *}(\mathscr{F})\right)(\mathfrak{T}) \rightarrow\left(F_{X / S_{0} *}(\mathscr{F})\right)(\rho(\mathfrak{Z})) \rightarrow\left(F_{X / S_{0} *}(\mathscr{F})\right)(\rho(\mathfrak{Z} \times \mathfrak{T} \mathfrak{Z})) .
$$


By (6.7.2), we deduce an $\mathscr{O}_{\mathfrak{T}}$-linear isomorphism $\mathscr{M} \stackrel{\sim}{\rightarrow}\left(F_{X / S_{0} *}(\mathscr{F})\right)_{\mathfrak{T}}$. In particular, $\left(F_{X / S_{0} *}(\mathscr{F})\right)_{\mathfrak{T}}$ is coherent. Hence $F_{X / S_{0} *}(\mathscr{F})$ is coherent.

Following the same argument as in the second part of the proof of ([34] 8.15), we show that for every morphism $g$ of $\operatorname{Conv}(X / \mathfrak{S})$, the transition morphism $c_{g}$ associated to $F_{X / S_{0} *}(\mathscr{F})$ is an isomorphism, i.e. $F_{X / S_{0} *}(\mathscr{F})$ is a crystal.

Proposition 6.8. We consider the following diagram (4.16):

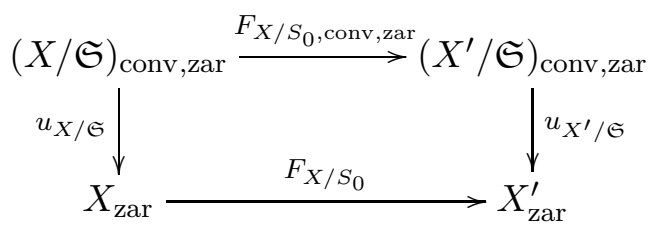

Let $\mathscr{E}$ be a convergent isocrystal of $(X / \mathrm{W})_{\text {conv,zar }}$ and denote the structure morphism $X \rightarrow S_{0}$ by $f$. Then there exists a canonical isomorphism in the derived category $\mathrm{D}\left(X_{\mathrm{zar}}, f^{-1}\left(\mathscr{O}_{\mathfrak{S}}\right)\right)$

$$
F_{X / S_{0} *}\left(\mathrm{R} u_{X / \mathfrak{S} *}(\mathscr{E})\right) \stackrel{\sim}{\rightarrow} \mathrm{R} u_{X^{\prime} / \mathfrak{S} *}\left(F_{X / S_{0} \operatorname{conv}, \mathrm{zar} *}(\mathscr{E})\right) .
$$

Proof. We consider $\mathscr{E}$ as a coherent crystal of $\mathscr{O}_{X / \mathfrak{S}}\left[\frac{1}{p}\right]$-module of $(X / \mathfrak{S})_{\text {conv,fppf }}$. Then, $\alpha_{*}(\mathscr{E})$ and $\mathscr{E}$ are equal as presheaves and $\mathrm{R}^{i} \alpha_{*}(\mathscr{E})=0$ for $i \geq 1$ (5.5). The assertion follows from 6.6 and the fact that $F_{X / S_{0}}: X_{\mathrm{zar}} \rightarrow X_{\mathrm{zar}}^{\prime}$ is an equivalence of topoi.

Corollary 6.9. Keep the assumption of 6.8 and suppose that there exists smooth liftings $\mathfrak{X}$ of $X$ and $\mathfrak{X}^{\prime}$ of $X^{\prime}$ over $\mathfrak{S}$. Let $f: X \rightarrow S_{0}$ be the canonical morphism. Then there exists a canonical isomorphism between the de Rham compelexes of $\mathscr{E}$ and of $F_{X / S_{0}, \operatorname{conv}, \mathrm{zar} *}(\mathscr{E})$ in $\mathrm{D}\left(X_{\mathrm{zar}}^{\prime}, f^{-1}\left(\mathscr{O}_{\mathfrak{S}}\right)\right)$

$$
F_{X / S_{0} *}\left(\mathscr{E}_{\mathfrak{X}} \otimes_{\mathscr{O}_{\mathfrak{X}}} \widehat{\Omega}_{\mathfrak{X} / \mathfrak{S}}^{\bullet}\right) \stackrel{\sim}{\rightarrow}\left(F_{X / S_{0}, \operatorname{conv}, \operatorname{zar} * \mathscr{E}}\right)_{\mathfrak{X}^{\prime}} \otimes_{\mathscr{O}_{\mathfrak{X}^{\prime}}} \widehat{\Omega}_{\mathfrak{X}^{\prime} / \mathfrak{S}}^{\bullet}
$$

Proof. It follows from (4.16.1) and 6.8.

Theorem 6.10. Let $g: X \rightarrow Y$ a smooth proper morphism of smooth $k$-schemes and $\mathscr{E}$ be a convergent isocrystal of $\operatorname{Conv}(X / \mathrm{W})_{\mathrm{conv}, \tau}$. Then $\mathrm{R}^{i} g_{\mathrm{conv}, \tau *}(\mathscr{E})$ is a convergent isocrystal of $\operatorname{Conv}(Y / \mathrm{W})_{\operatorname{conv}, \tau}$ for every $i \geq 0$.

Inspired by Ogus' arguments in [26, we use 5.10 and Dwork's trick to prove 6.10. To do this, we introduce certain subcategories of $\operatorname{Conv}(X / \mathrm{W})$.

Definition 6.11. (i) Let $n$ be an integer $\geq 0$ and $T$ a $k$-scheme. We denote by $T^{(n)}$ the closed subscheme of $T$ defined by the ideal sheaf $\left\{x \in \mathscr{O}_{T} \mid x^{p^{n}}=0\right\}$.

(ii) We denote by $\operatorname{Conv}^{(n)}(X / \mathrm{W})$ the full subcategory of $\operatorname{Conv}(X / \mathrm{W})$ consisting of objects $(\mathfrak{T}, u)$ such that $u: T_{0} \rightarrow X$ can be lifted to a $k$-morphism $\widetilde{u}: T^{(n)} \rightarrow X$.

Given an object $(\mathfrak{T}, u)$ of $\operatorname{Conv}^{(n)}(X / \mathrm{W})$ and a morphism $\left(\mathfrak{T}^{\prime}, u^{\prime}\right) \rightarrow(\mathfrak{T}, u)$ of $\operatorname{Conv}(X / \mathrm{W})$, then $\left(\mathfrak{T}^{\prime}, u^{\prime}\right)$ is also an object of $\operatorname{Conv}^{(n)}(X / \mathrm{W})$. In particular, $T^{(0)}=T$ and $\operatorname{Conv}^{(0)}(X / \mathrm{W})$ coincides with $\mathrm{pConv}(X / \mathrm{W})$ (5.7).

Lemma 6.12. The functor $\rho$ (6.1.2) sends $\operatorname{Conv}^{(n+1)}(X / \mathrm{W})$ to $\operatorname{Conv}^{(n)}\left(X^{\prime} / \mathrm{W}\right)$.

Proof. Let $(\mathfrak{T}, u)$ be an object of $\operatorname{Conv}^{(n+1)}(X / \mathrm{W})$ and $\widetilde{u}: T^{(n+1)} \rightarrow X$ a lifting of $u$. The absolute Frobenius morphism $T^{(n)} \rightarrow T^{(n)}$ factors through the closed subscheme $T^{(n+1)}$ and then the relative 
Frobenius morphism $F_{T^{(n)} / k}$ factors through $\left(T^{(n+1)}\right)^{\prime}$. We have a commutative diagram

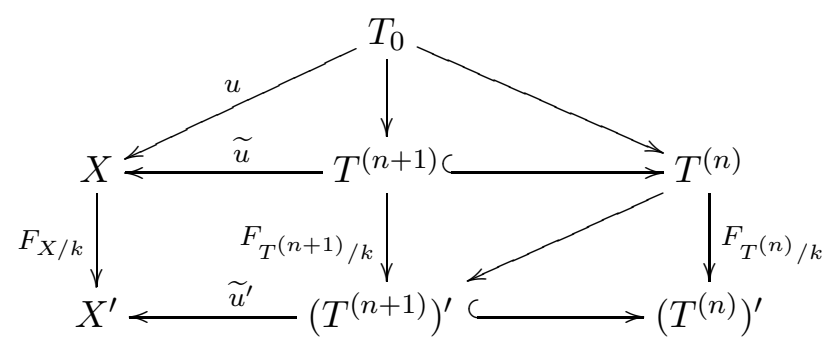

Then the morphism $F_{X / k} \circ u$ can be lifted to a $k$-morphism $T^{(n)} \rightarrow X^{\prime}$ and the lemma follows.

6.13. Proof of 6.10. By [5.5, it suffices to prove the assertion for fppf topology. There exists a convergent isocrystal $\mathscr{G}$ on $\operatorname{Conv}\left(X^{\prime} / \mathrm{W}\right)$ with $F_{X / k, \text { conv,fppf }}^{*}(\mathscr{G}) \simeq \mathscr{E}$ (6.7). If we set $\mathscr{F}=$ $\mathrm{R}^{i} g_{\text {conv }, \text { fpp } *}(\mathscr{E}), g^{\prime}=g \otimes_{k, \sigma} k$ and $\mathscr{H}=\mathrm{R}^{i} g_{\text {conv }, \mathrm{fppf} *}^{\prime}(\mathscr{G})$, then we have $F_{Y / k, \text { conv,fppf }}^{*}(\mathscr{H}) \simeq \mathscr{F}$ by 6.6 .

Each object (resp. morphism) of $\operatorname{Conv}(Y / \mathrm{W})$ belongs to a subcategory $\operatorname{Conv}^{(n)}(Y / \mathrm{W})$ (6.11) for some integer $n$. We prove the following assertions by induction:

(i) For every object $\mathfrak{T}$ of $\operatorname{Conv}^{(n)}(Y / \mathrm{W}), \mathscr{F}_{\mathfrak{T}}$ is coherent.

(ii) For every morphism $f$ of $\operatorname{Conv}^{(n)}(Y / \mathrm{W})$, the transition morphism $c_{f}$ associated to $\mathscr{F}$ is an isomorphism.

Assertions for $n=0$ are proved in 5.10. Suppose that assertions hold for $n \geq 0$ and we prove them for $n+1$. Let $(\mathfrak{T}, u)$ be an object of $\operatorname{Conv}^{(n+1)}(Y / \mathrm{W})$. By (4.10.2), we deduce that

$$
\mathscr{H}_{\rho(\mathfrak{T})} \stackrel{\sim}{\rightarrow} \mathscr{F}_{\mathfrak{T}} .
$$

By induction hypotheses, for any object $\mathfrak{Z}$ of $\operatorname{Conv}^{(n)}\left(X^{\prime} / \mathrm{W}\right), \mathscr{H}_{\mathfrak{Z}}$ is coherent. Then assertion (i) follows from 6.12 and the induction hypotheses.

Assertion (ii) can be verified in the same way by 4.10 and 6.12 .

6.14. We denote by $\sigma: \mathrm{W} \rightarrow \mathrm{W}$ the Frobenius homomorphism. It induces a morphism of topoi $\left(X^{\prime} / \mathrm{W}\right)_{\mathrm{conv}, \tau} \rightarrow(X / \mathrm{W})_{\mathrm{conv}, \tau}$ by functoriality. For any sheaf $\mathscr{E}$ of $(X / \mathrm{W})_{\mathrm{conv}, \tau}$, we denote by $\mathscr{E}^{\prime}$ the inverse image of $\mathscr{E}$ to $\left(X^{\prime} / \mathrm{W}\right)_{\text {conv }, \tau}$.

For $\tau \in\{$ zar, fppf $\}$, a convergent $F$-isocrystal of $\operatorname{Conv}(X / \mathrm{W})_{\operatorname{conv}, \tau}$ is a pair $(\mathscr{E}, \varphi)$ consisting of a convergent isocrystal $\mathscr{E}$ of $(X / \mathrm{W})_{\text {conv }, \tau}$ and an isomorphism, called Frobenius structure

$$
\varphi: F_{X / k, \mathrm{conv}, \tau}^{*}\left(\mathscr{E}^{\prime}\right) \stackrel{\sim}{\rightarrow} \mathscr{E} .
$$

Corollary 6.15. Keep the assumption of 6.10 and let $\varphi$ be a Frobenius structure on $\mathscr{E}$. Then, for any $i \geq 0$, the pair $\left(\mathrm{R}^{i} g_{\mathrm{conv}, \tau *}(\mathscr{E}), \mathrm{R}^{i} g_{\mathrm{conv}, \tau *}(\varphi)\right)$ is a convergent $F$-isocrystal of $\operatorname{Conv}(Y / \mathrm{W})_{\operatorname{conv}, \tau}$.

Proof. Consider the isomorphism

$$
\mathrm{R}^{i} g_{\text {conv }, \text { fppf } *}(\varphi): \mathrm{R}^{i} g_{\text {conv }, \text { fppf } *}\left(F_{X / k, \text { conv }, \text { fppf }}^{*}\left(\mathscr{E}^{\prime}\right)\right) \stackrel{\sim}{\rightarrow} \mathrm{R}^{i} g_{\text {conv }, f p p f}(\mathscr{E}) .
$$

By 5.6 and 6.6, the left hand side is isomorphic to $F_{Y / k, \text { conv,fppf }}^{*}\left(\left(\mathrm{R}^{i} g_{\text {conv,fppf } *}(\mathscr{E})\right)^{\prime}\right)$. Then the assertion follows. 


\section{REVIEW ON RIGID GEOMETRY}

7.1. Recall that $\mathbf{S}$ denotes the category of formal W-scheme of finite type whose morphisms are Wmorphisms of finite type (2.1). The set $\mathbf{B}$ of admissible blow-ups in $\mathbf{S}$ forms a right multiplicative system in $\mathbf{S}$ (1] 4.1.4). We denote by $\mathbf{R}$ the localized category of $\mathbf{S}$ relative to $\mathbf{B}$. Objects of $\mathbf{R}$ are called coherent rigid spaces (over $K=\mathrm{W}\left[\frac{1}{p}\right]$ ). For any object $\mathfrak{X}$ (resp. morphism $f$ ) of $\mathbf{S}$, its image in $\mathbf{R}$ is denoted by $\mathfrak{X}^{\text {rig }}$ (resp. $f^{\text {rig }}$ ).

For any object $\mathfrak{X}$ of $\mathbf{S}$, we set $\mathbf{B}_{\mathfrak{X}}$ the full subcategory of $\mathbf{S}_{/ \mathfrak{X}}$ consisting of admissible blowups.

7.2. Recall that $\mathbf{S}^{\diamond}$ denotes the full subcategory of $\mathbf{S}$ consisting of flat formal $\mathrm{W}$-schemes of finite type (2.1). For any object $\mathfrak{X}$ of $\mathbf{S}^{\diamond}$ and any admissible blow-up $\varphi: \mathfrak{X}^{\prime} \rightarrow \mathfrak{X}, \mathfrak{X}^{\prime}$ is still an object of $\mathbf{S}^{\diamond}$ (1] 3.1.4). Then the set $\mathbf{B}^{\diamond}$ of admissible blow-ups in $\mathbf{S}^{\diamond}$ forms a right multiplicative system in $\mathbf{S}^{\diamond}$. By ([1] 4.1.15(iii)), the canonical functor $\mathbf{S}^{\diamond} \rightarrow \mathbf{R}$ is essentially surjective and hence induces an equivalence of categories between the localized category of $\mathbf{S}^{\diamond}$ relative to $\mathbf{B}^{\diamond}$ and $\mathbf{R}$.

7.3. Let $\mathcal{X}$ be a coherent rigid space. We denote by $\langle\mathcal{X}\rangle$ the set of rigid points of $\mathcal{X}([1] 4.3 .1)$. We say that a family of morphisms $\left(f_{i}: \mathcal{X}_{i} \rightarrow \mathcal{X}\right)_{i \in I}$ is a covering for rigid points if $\bigcup_{i \in I} f_{i}\left(\left\langle\mathcal{X}_{i}\right\rangle\right)=\langle\mathcal{X}\rangle$.

Recall ([1] 4.3.8) that a family $\left(\mathcal{X}_{i} \rightarrow \mathcal{X}\right)_{i \in I}$ of open immersions of coherent rigid spaces ([1] 4.2.1) is an admissible covering if it admits a finite sub-covering for rigid points. We denote by $\mathbf{A d}_{/ \mathcal{X}}$ the full subcategory of $\mathbf{R}_{/ \mathcal{X}}$ consisting of open immersions to $\mathcal{X}$ and by $\mathcal{X}_{\text {ad }}$ the topos of sheaves of sets on $\mathbf{A d}_{/ \mathcal{X}}$ for the admissible topology.

7.4. In the following of this section, $\mathfrak{X}$ denotes an object of $\mathbf{S}$. The functor $\mathbf{Z a r}_{/ \mathfrak{X}} \rightarrow \mathbf{A d}_{/ \mathfrak{X}^{\text {rig }}}$ defined by $\mathfrak{U} \mapsto \mathfrak{U}^{\text {rig }}$ is continuous and left exact and induces a morphism of topoi ([1] 4.5.2)

$$
\rho_{\mathfrak{X}}: \mathfrak{X}_{\mathrm{ad}}^{\mathrm{rig}} \rightarrow \mathfrak{X}_{\mathrm{zar}} .
$$

For any object $\left(\mathfrak{X}^{\prime}, \varphi\right)$ of $\mathbf{B}_{\mathfrak{X}}$, we denote by $\mu_{\varphi}$ the composition

$$
\mu_{\varphi}: \mathfrak{X}_{\mathrm{ad}}^{\mathrm{rig}} \stackrel{\sim}{\rightarrow} \mathfrak{X}_{\mathrm{ad}}^{\prime \text { rig }} \stackrel{\rho_{\mathfrak{X}^{\prime}}}{\longrightarrow} \mathfrak{X}_{\text {zar }}^{\prime} .
$$

Let $\mathscr{F}$ be an $\mathscr{O}_{\mathfrak{X}}$-module. We denote by $\mathscr{F}^{\text {rig }}$ the rigid fiber associated to $\mathscr{F}$ ([1] 4.7.4) which is a sheaf of $\mathfrak{X}_{\mathrm{ad}}^{\mathrm{rig}}$. We have a functorial isomorphism ([1] 4.7.4.2)

$$
\mathscr{F}^{\text {rig }} \stackrel{\sim}{\rightarrow} \underset{\left(\mathfrak{X}^{\prime}, \varphi\right) \in \mathbf{B}_{\mathfrak{X}}^{\circ}}{\lim } \mu_{\varphi}^{*}\left(\left(\varphi_{\mathrm{zar}}^{*}(\mathscr{F})\right)\left[\frac{1}{p}\right]\right) .
$$

In particular, $\left(\mathscr{O}_{\mathfrak{X}}\right)^{\text {rig }}$ is a ring that we also denote by $\mathscr{O}_{\mathfrak{X}}$ rig. We have canonical morphisms of ringed topoi ([1] 4.7.5)

$$
\left.\rho_{\mathfrak{X}}:\left(\mathfrak{X}_{\mathrm{ad}}^{\mathrm{rig}}, \mathscr{O}_{\mathfrak{X}^{\mathrm{rig}}}\right) \rightarrow\left(\mathfrak{X}_{\text {zar }}, \mathscr{O}_{\mathfrak{X}}\left[\frac{1}{p}\right]\right), \quad \mu_{\varphi}:\left(\mathfrak{X}_{\mathrm{ad}}^{\mathrm{rig}}, \mathscr{O}_{\mathfrak{X}^{\mathrm{rig}}}\right) \stackrel{\rho_{\mathfrak{X}^{\prime}}}{\longrightarrow}\left(\mathfrak{X}_{\text {zar }}^{\prime}, \mathscr{O}_{\mathfrak{X}^{\prime}}\left[\frac{1}{p}\right]\right)\right) .
$$

If $\mathscr{F}$ is moreover coherent, we have a canonical isomorphism $\rho_{\mathfrak{X}}^{*}\left(\mathscr{F}\left[\frac{1}{p}\right]\right) \stackrel{\sim}{\rightarrow} \mathscr{F}$ rig ([1] 4.7.2.8).

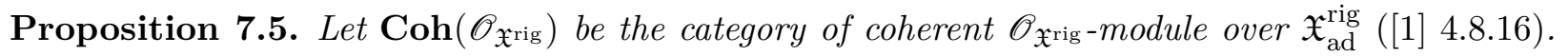
The inverse image and direct image functors of $\rho_{\mathfrak{X}}$ induces equivalences of categories quasi-inverse to each other

$$
\operatorname{Coh}\left(\mathscr{O}_{\mathfrak{X}}\left[\frac{1}{p}\right]\right) \rightleftharpoons \operatorname{Coh}\left(\mathscr{O}_{\mathfrak{X}^{\text {rig }}}\right)
$$


Proof. We write simply $\rho$ for $\rho_{\mathfrak{X}}$. By ([1] 4.7.8.1), $\rho_{*}$ send coherent $\mathscr{O}_{\mathfrak{X}}$ rig-modules to coherent $\mathscr{O}_{\mathfrak{X}}\left[\frac{1}{p}\right]$-modules. By (2.4.2) and ([1] 4.8.18), the inverse image functor $\rho^{*}$ is essentially surjective. By (1] 4.7.8.2 and 4.7.29.2), $\rho^{*}$ is fully faithful. In view of the canonical isomorphisms $\rho^{*} \stackrel{\sim}{\rightarrow} \rho^{*} \rho_{*} \rho^{*}$ and $\rho^{*} \rho_{*} \rho^{*} \stackrel{\sim}{\rightarrow} \rho^{*}$, we deduce that $\rho_{*}$ is a quasi-inverse.

7.6. Let $f: \mathfrak{X} \rightarrow \mathfrak{Y}$ be a morphism of $\mathbf{S}$. It induces a morphism of ringed topoi $f_{\mathrm{ad}}^{\text {rig }}:\left(\mathfrak{X}_{\mathrm{ad}}^{\text {rig }}, \mathscr{O}_{\mathfrak{X}^{\text {rig }}}\right) \rightarrow$ $\left(\mathfrak{Y}_{\mathrm{ad}}^{\mathrm{rig}}, \mathscr{O}_{\mathfrak{Y}}\right.$ rig $)([1] 4.7 .2 .1)$. The diagram

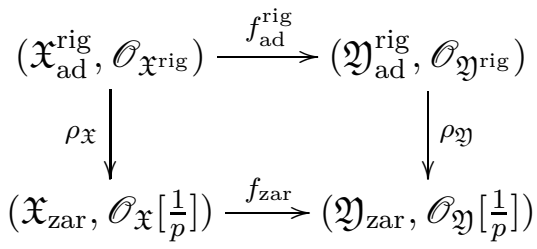

is commutative up to canonical isomorphisms ([1 4.7.24.2).



$$
\rho_{\mathfrak{X}}^{*}\left(f_{\mathrm{zar}}^{*}\left(\rho_{\mathfrak{Y} *}(\mathscr{F})\right)\right) \stackrel{\sim}{\rightarrow} f_{\mathrm{ad}}^{\mathrm{rig} *}\left(\rho_{\mathfrak{Y}}^{*}\left(\rho_{\mathfrak{Y} *}(\mathscr{F})\right)\right) \stackrel{\sim}{\rightarrow} f_{\mathrm{ad}}^{\text {rig } *}(\mathscr{F}) .
$$

Then we deduce that the following base change morphism is an isomorphism

$$
f_{\mathrm{zar}}^{*} \rho_{\mathfrak{Y} *}(\mathscr{F}) \stackrel{\sim}{\rightarrow} \rho_{\mathfrak{X} *} f_{\mathrm{ad}}^{\mathrm{rig} *}(\mathscr{F}) .
$$

7.7. Following ([1 5.10.1), we say that a morphism $f: \mathcal{X} \rightarrow \mathcal{Y}$ of coherent rigid spaces is flat if the associated morphism of ringed topoi $f_{\mathrm{ad}}:\left(\mathcal{X}_{\mathrm{ad}}, \mathscr{O}_{\mathcal{X}}\right) \rightarrow\left(\mathcal{Y}_{\mathrm{ad}}, \mathscr{O}_{\mathcal{Y}}\right)$ is flat $([3] \mathrm{V} 1.7)$. We say that $f$ is faithfully flat if $f$ is flat and $f(\langle\mathcal{X}\rangle)=\langle\mathcal{Y}\rangle$ ([1] 4.3.1 and 5.10.11).

A morphism $f: \mathfrak{X} \rightarrow \mathfrak{Y}$ of $\mathbf{S}$ is rig-flat (2.7) if and only if $f^{\text {rig }}$ is flat ([1 5.5.8).

We say that a family $\left(\mathcal{X}_{i} \rightarrow \mathcal{X}\right)_{i \in I}$ of flat morphisms of $\mathbf{R}$ is a fppf covering if it admits a finite sub-covering for rigid points (7.3). In view of ([1 5.10.12), fppf coverings are stable by composition and by base change in $\mathbf{R}$.

Let $\mathcal{X}$ be a coherent rigid space. We denote by $\mathbf{R} \mathbf{f}_{/ \mathcal{X}}$ the full subcategory of $\mathbf{R}_{/ \mathcal{X}}$ consisting of flat morphisms to $\mathcal{X}$. We call fppf topology the topology on $\mathbf{R f}_{/ \mathcal{X}}$ generated by the pretopology for which coverings are fppf coverings. We denote by $\mathcal{X}_{\text {fppf }}$ the topos of sheaves of sets on this site.

By fppf descent of morphisms ([1] 5.12.4), the fppf topology on $\mathbf{R f} / \mathcal{X}$ is sub-canonical, i.e. the presheaf associated to each object of $\mathbf{R f}_{/ \mathcal{X}}$ is a sheaf for the fppf topology.

7.8. The canonical functor $\mathbf{A d}_{/ \mathcal{X}} \rightarrow \mathbf{R f}_{/ \mathcal{X}}$ is continuous and left exact. Then it induces a morphism of topoi

$$
\alpha_{\mathcal{X}}: \mathcal{X}_{\mathrm{fppf}} \rightarrow \mathcal{X}_{\mathrm{ad}}
$$

Given a morphism $f: \mathcal{X}^{\prime} \rightarrow \mathcal{X}$ of $\mathbf{R}$, the canonical functor $\mathbf{R f}_{/ \mathcal{X}} \rightarrow \mathbf{R f}_{/ \mathcal{X}^{\prime}}\left(\operatorname{resp} . \mathbf{A d}_{/ \mathcal{X}} \rightarrow\right.$ $\left.\operatorname{Ad}_{/ \mathcal{X}^{\prime}}\right)$ defined by $\mathcal{Y} \mapsto \mathcal{Y} \times \mathcal{X} \mathcal{X}^{\prime}$ is continuous and left exact. It induces morphisms of topoi

$$
f_{\tau}: \mathcal{X}_{\tau}^{\prime} \rightarrow \mathcal{X}_{\tau}, \quad \tau \in\{\mathrm{ad}, \mathrm{fppf}\},
$$

compatible with $\alpha_{\mathcal{X}}$ and $\alpha_{\mathcal{X}}$. If $f$ is a morphism of $\mathbf{R f}_{/ \mathcal{X}}(\operatorname{resp} . \mathbf{A d} / \mathcal{X})$, in view of the description of direct image functors, one verifies that the above morphism coincides with the localization morphism at $\mathcal{X}^{\prime}$. 
7.9. Let $\mathscr{F}$ be a coherent $\mathscr{O}_{\mathcal{X}}$-module. The presheaf on $\mathbf{R f}_{/ \mathcal{X}}$

$$
\left(f: \mathcal{X}^{\prime} \rightarrow \mathcal{X}\right) \mapsto \Gamma\left(\mathcal{X}^{\prime}, f_{\mathrm{ad}}^{*}(\mathscr{F})\right)
$$

is a sheaf for the fppf topology by fppf descent for coherent modules on rigid spaces (1 1 5.11.11). In particular, $\mathscr{O}_{\mathcal{X}}$ defines a sheaf of rings of $\mathcal{X}_{\text {fppf }}$ that we still denote by $\mathscr{O}_{\mathcal{X}}$. We call abusively coherent $\mathscr{O}_{\mathcal{X}}$-module of $\mathcal{X}_{\text {fppf }}$ a sheaf of $\mathcal{X}_{\text {fppf }}$ associated to a coherent $\mathscr{O}_{\mathcal{X}}$-module of $\mathcal{X}_{\text {ad }}$.

For $\tau \in\{$ ad,fppf $\}$, the morphism of topoi $f_{\tau}(7.8 .2)$ is ringed by $\mathscr{O}_{\mathcal{X}}$ and $\mathscr{O}_{\mathcal{X}^{\prime}}$. For any $\mathscr{O}_{\mathcal{X}}$ module $\mathscr{M}$ of $\mathcal{X}_{\tau}$, we use $f_{\tau}^{-1}(\mathscr{M})$ to denote the inverse image in the sense of sheaves and we keep $f_{\tau}^{*}(\mathscr{M})$ for the inverse image in the sense of modules.

\section{RIGID CONVERGENT TOPOS AND CONVERGENT ISOCRYSTALS}

8.1. In this section, $\mathfrak{S}$ denotes a flat formal $\mathrm{W}$-scheme of finite type and $X$ an $S$-scheme.

We will introduce a full subcategory of $(X / \mathfrak{S})_{\text {conv,zar }}$ consisting of sheaves $\mathscr{F}=\left\{\mathscr{F}_{\mathfrak{T}}, \beta_{f}\right\}$ (4.5) such that the morphism $\beta_{f}$ is an isomorphism if the underlying morphism of formal schemes of $f$ is an admissible blowup. It turns out that this category forms a topos $(X / \mathfrak{S})_{\text {rconv,ad }}(8.3$, 8.13) and


and their cohomologies remain unchanged in this topos (8.19, 8.25).

We begin by introducing $(X / \mathfrak{S})_{\text {rconv,ad }}$ and its fppf variant.

Lemma 8.2. We denote by $\mathbf{B}_{X / \mathfrak{S}}$ the set of morphisms in $\operatorname{Conv}(X / \mathfrak{S})$ (4.1) whose underlying morphism on formal schemes is an admissible blow-up. Then, it forms a right multiplicative system in $\operatorname{Conv}(X / \mathfrak{S})$.

Proof. For any object $(\mathfrak{T}, u)$ of $\operatorname{Conv}(X / \mathfrak{S})$, we have a canonical functor $s_{\mathfrak{T}}: \mathbf{B}_{\mathfrak{T}} \rightarrow \operatorname{Conv}(X / \mathfrak{S})$ sending $\left(\mathfrak{T}^{\prime}, \varphi\right)$ to $\left(\mathfrak{T}^{\prime}, u \circ \varphi_{0}\right)$. Then the assertion follow from the facts that admissible blow-ups form a right multiplicative system in $\mathbf{S}_{/ \mathfrak{S}}^{\diamond}(7.2)$ and that the canonical functor $\operatorname{Conv}(X / \mathfrak{S}) \rightarrow \mathbf{S}_{\mathfrak{S}}^{\diamond}$ is faithful.

8.3. We denote by $\operatorname{RConv}(X / \mathfrak{S})$ the localized category of $\operatorname{Conv}(X / \mathfrak{S})$ relative to $\mathbf{B}_{X / \mathfrak{S} \text {. More }}$ precisely, objects of $\operatorname{RConv}(X / \mathfrak{S})$ are same as those of $\operatorname{Conv}(X / \mathfrak{S})$. For two objects $(\mathfrak{Z}, v),(\mathfrak{T}, u)$ of $\operatorname{RConv}(X / \mathfrak{S})$, we have

$$
\operatorname{Hom}_{\mathrm{RConv}(X / \mathfrak{S})}((\mathfrak{Z}, v),(\mathfrak{T}, u))=\underset{\left(\mathfrak{Z}^{\prime}, \vec{\varphi}\right) \in \mathbf{B}_{\mathfrak{Z}}^{\circ}}{\lim _{\longrightarrow}} \operatorname{Hom}_{\operatorname{Conv}(X / \mathfrak{S})}\left(\left(\mathfrak{Z}^{\prime}, v \circ \varphi_{0}\right),(\mathfrak{T}, u)\right) .
$$

We denote by $\mathrm{Q}_{X / \mathfrak{S}}$ the canonical functor

$$
\mathrm{Q}_{X / \mathfrak{S}}: \operatorname{Conv}(X / \mathfrak{S}) \rightarrow \operatorname{RConv}(X / \mathfrak{S})
$$

For an object $\mathfrak{T}$ (resp. a morphism $f$ ) of $\operatorname{Conv}(X / \mathfrak{S})$, we write $\mathfrak{T}^{\text {rig }}=Q_{X / \mathfrak{S}}(\mathfrak{T})$ (resp. $f^{\text {rig }}=$ $\left.\mathrm{Q}_{X / \mathfrak{S}}(f)\right)$, if there is no risk of confusion.

8.4. We denote by $\widehat{\operatorname{Conv}}(X / \mathfrak{S})(\operatorname{resp} . \widehat{\operatorname{RConv}}(X / \mathfrak{S}))$ the category of presheaves on $\operatorname{Conv}(X / \mathfrak{S})$ $($ resp. $\operatorname{RConv}(X / \mathfrak{S}))$ and by $\mathrm{Q}_{X / \mathfrak{S}}^{*}: \widehat{\operatorname{RConv}}(X / \mathfrak{S}) \rightarrow \widehat{\operatorname{Conv}}(X / \mathfrak{S})$ the functor defined by $\mathscr{F} \mapsto$ $\mathscr{F} \circ \mathrm{Q}_{X / \mathfrak{S}}$. The functor $\mathrm{Q}_{X / \mathfrak{S} *}$ admits a left adjoint $\mathrm{Q}_{X / \mathfrak{S} !}$ ([3] I 5.1) defined as follows.

For any object $\mathfrak{T}^{\text {rig }}$ of $\operatorname{RConv}(X / \mathfrak{S})$, we denote by $I_{\mathrm{Q}}^{\mathfrak{T}^{\text {rig }}}$ the category whose object are pairs $(\mathfrak{Z}, g)$ consisting of an object $\mathfrak{Z}$ of $\operatorname{Conv}(X / \mathfrak{S})$ and a morphism $g: \mathfrak{T}^{\text {rig }} \rightarrow \mathfrak{Z}^{\text {rig }}$ of $\operatorname{RConv}(X / \mathfrak{S})$. A 
morphism $\left(\mathfrak{Z}^{\prime}, g^{\prime}\right) \rightarrow(\mathfrak{Z}, g)$ is given by a morphism $\mu: \mathfrak{Z}^{\prime} \rightarrow \mathfrak{Z}$ of $\operatorname{Conv}(X / \mathfrak{S})$ such that $g=\mu^{\text {rig }} \circ g^{\prime}$. Then we have ([3] I 5.1.1)

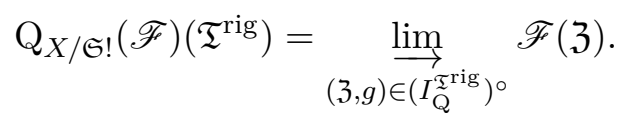

Moreover, we have a commutative diagram ([3] I 1.5.4)



where the vertical functors are the canonical functors.

Proposition 8.5. (i) The category $\left(I_{\mathrm{Q}}^{\mathfrak{T} \text { rig }}\right)^{\circ}$ is filtered ([3] I 2.7).

(ii) The functor $\mathrm{Q}_{X / \mathfrak{S} \text { ! }}$ is left exact (and hence is exact).

(iii) Fiber products are representable in $\operatorname{RConv}(X / \mathfrak{S})$ and $\mathrm{Q}_{X / \mathfrak{S}}$ commutes with fiber products.

Proof. We verify following conditions of ([3] I 2.7) for $\left(I_{\mathrm{Q}}^{\mathfrak{T}^{\text {rig }}}\right)^{\circ}$ :

(PS1) Given two morphisms $u:\left(\mathfrak{Z}_{1}, g_{1}\right) \rightarrow\left(\mathfrak{Z}_{0}, g_{0}\right)$ and $v:\left(\mathfrak{Z}_{2}, g_{2}\right) \rightarrow\left(\mathfrak{Z}_{0}, g_{0}\right)$ of $I_{\mathrm{Q}}^{\text {rig }}$, by (8.3.1) and (8.2), there exists an admissible blow-up $\mathfrak{T}^{\prime}$ of $\mathfrak{T}$ and morphisms $\mathfrak{g}_{i}: \mathfrak{T}^{\prime} \rightarrow \mathfrak{Z}_{i}$ of $\operatorname{Conv}(X / \mathfrak{S})$ such that $\mathfrak{g}_{i}^{\text {rig }}=g_{i}$ and that $u \circ \mathfrak{g}_{1}=\mathfrak{g}_{0}=v \circ \mathfrak{g}_{2}$. Then we obtain a morphism $\mathfrak{h}: \mathfrak{T}^{\prime} \rightarrow \mathfrak{Z}_{1} \times \mathfrak{Z}_{0} \mathfrak{Z}_{2}$ of $\operatorname{Conv}(X / \mathfrak{S})$ (4.2) and an object $\left(\mathfrak{Z}_{1} \times \mathfrak{Z}_{0} \mathfrak{Z}_{2}, \mathfrak{h}^{\text {rig }}\right)$ of $I_{\mathrm{Q}}^{\text {rig }}$ dominant $\left(\mathfrak{Z}_{i}, g_{i}\right)$ for $i=1,2$. The diagram

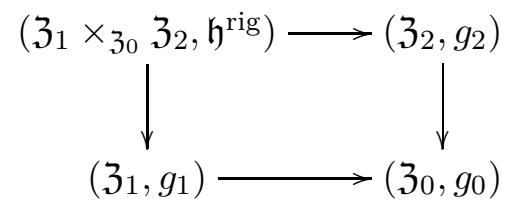

commutes. Then condition (PS1) follows.

(PS2) Let $u, v:(\mathfrak{Y}, g) \rightarrow(\mathfrak{Z}, h)$ be two morphisms of $I_{\widehat{Q}}^{\mathfrak{T}^{\text {rig }}}$. There exists an admissible blow-up $\left(\mathfrak{T}^{\prime}, \varphi\right)$ of $\mathfrak{T}$ and a morphism $\mathfrak{g}: \mathfrak{T}^{\prime} \rightarrow \mathfrak{Y}$ of $\operatorname{Conv}(X / \mathfrak{S}) \operatorname{such}$ that $u \circ \mathfrak{g}=v \circ \mathfrak{g}$ in $\operatorname{Conv}(X / \mathfrak{S})$, denoted by $\mathfrak{h}$. We have $\mathfrak{g}^{\text {rig }}=g, \mathfrak{h}^{\text {rig }}=h$. Then $\left(\mathfrak{T}^{\prime}, \varphi^{\text {rig }}\right)$ defines an object of $I_{\mathbb{Q}}^{\mathfrak{T}^{\text {rig }}}$ and $\mathfrak{h}($ resp. $\mathfrak{g})$ defines morphism from $\left(\mathfrak{T}^{\prime}, \varphi^{\text {rig }}\right)$ to $(\mathfrak{Y}, g)$ (resp. $(\mathfrak{Z}, h)$ ). Condition (PS2) follows from $u \circ \mathfrak{g}=v \circ \mathfrak{g}=\mathfrak{h}$.

It is clear that $I_{\mathrm{Q}}^{\mathfrak{T}^{\text {rig }}}$ is non-empty. Given two objects $\left(\mathfrak{Z}_{1}, g_{1}\right)$ and $\left(\mathfrak{Z}_{2}, g_{2}\right)$, there exists an admissible blow-up $\mathfrak{T}^{\prime}$ of $\mathfrak{T}$ and morphisms $\mathfrak{g}_{i}: \mathfrak{T}^{\prime} \rightarrow \mathfrak{Z}_{i}$ of $\operatorname{Conv}(X / \mathfrak{S})$ for $i=1,2$ such that $\mathfrak{g}_{i}^{\text {rig }}=g_{i}$. Hence, $I_{\mathrm{Q}}^{\mathfrak{T} \text { rig }}$ is connected. Then assertion (i) follows.

Assertion (ii) follows from (i). Assertion (iii) follows from (ii), (8.4.2) and the fact that fiber product is representable in $\operatorname{Conv}(X / \mathfrak{S})$.

8.6. The canonical functor $\operatorname{Conv}(X / \mathfrak{S}) \rightarrow \mathbf{S}_{/ \mathfrak{S}}^{\diamond}$ defined by $(\mathfrak{T}, u) \mapsto \mathfrak{T}$ induces a functor

$$
\operatorname{RConv}(X / \mathfrak{S}) \rightarrow \mathbf{R}_{/ \mathfrak{S}^{\text {rig }}} .
$$

In view of the definition of fiber product in $\mathbf{R}_{/ \mathfrak{S}^{\text {rig }}}\left(\left[\begin{array}{l}1 \\ \text { 4.1.13), the above functor commutes with }\end{array}\right.\right.$ fiber products. 
8.7. We say that a family of morphisms $\left\{\left(\mathfrak{T}_{i}, u_{i}\right)^{\text {rig }} \rightarrow(\mathfrak{T}, u)^{\text {rig }}\right\}_{i \in I}$ of $\operatorname{RConv}(X / \mathfrak{S})$ is an admissible (resp. fppf) covering if its image in its image $\left\{\mathfrak{T}_{i}^{\text {rig }} \rightarrow \mathfrak{T}^{\text {rig }}\right\}_{i \in I}$ in $\mathbf{R}$ is an admissible (resp. fppf) covering (7.3, 7.7). By 7.7 and 8.6, admissible (resp. fppf) coverings form a pretopology. For $\tau=$ ad (resp. fppf), we call rigid convergent topos of $X$ over $\mathfrak{S}$ and denote by $(X / \mathfrak{S})_{\mathrm{rconv}, \tau}$ the topos of sheaves of sets on $\operatorname{RConv}(X / \mathfrak{S})$, equipped with the topology associated to the pretopology defined by admissible (resp. fppf) coverings.

8.8. Let $(\mathfrak{T}, u)$ be an object of $\operatorname{Conv}(X / \mathfrak{S})$. The canonical functor (7.2)

$$
r_{\mathfrak{T}}: \mathbf{S}_{/ \mathfrak{T}}^{\diamond} \rightarrow \operatorname{Conv}(X / \mathfrak{S}) \quad\left(f: \mathfrak{T}^{\prime} \rightarrow \mathfrak{T}\right) \mapsto\left(\mathfrak{T}^{\prime}, u \circ f_{0}\right)
$$

sends admissible blow-ups to $\mathbf{B}_{X / \mathfrak{S}}$ and hence induces a functor

$$
r_{\mathfrak{T}^{\text {rig }}}: \mathbf{R}_{/ \mathfrak{T}^{\text {rig }}} \rightarrow \operatorname{RConv}(X / \mathfrak{S}) .
$$

The restriction of 8.8 .1$)$ to $\mathbf{A d}_{/ \mathfrak{T}^{\text {rig }}}\left(\mathrm{resp} . \mathbf{R f}_{/ \mathfrak{T}^{\mathrm{rig}}}\right.$ ) is cocontinuous for the admissible (resp. fppf) topology and it induces a morphism of topoi

$$
s_{\mathfrak{T}^{\text {rig }}}: \mathfrak{T}_{\tau}^{\text {rig }} \rightarrow(X / \mathfrak{S})_{\mathrm{conv}, \tau}, \quad \tau \in\{\mathrm{ad}, \mathrm{fppf}\} .
$$

For any sheaf $\mathscr{F}$ of $(X / \mathfrak{S})_{\text {conv }, \tau}$, we set $\mathscr{F}_{\mathfrak{T}^{\text {rig }}}=s_{\mathfrak{T}^{\text {rig }}}^{*}(\mathscr{F})$. For any morphism $f: \mathfrak{T}^{\prime \text { rig }} \rightarrow \mathfrak{T}^{\text {rig }}$ of $\operatorname{RConv}(X / \mathfrak{S})$, we have a canonical morphism

$$
\mathscr{F}_{\mathfrak{T}^{\text {rig }}} \rightarrow f_{\tau *}\left(\mathscr{F}_{\mathfrak{T}^{\prime} \text { rig }}\right)
$$

and we denote its adjoint by

$$
\gamma_{f}: f_{\tau}^{*}\left(\mathscr{F}_{\mathfrak{T}^{\text {rig }}}\right) \rightarrow \mathscr{F}_{\mathfrak{T}^{\prime}} \text { rig },
$$

where $f_{\tau}: \mathfrak{T}_{\tau}^{\prime \text { rig }} \rightarrow \mathfrak{T}_{\tau}^{\text {rig }}$ denotes the functorial morphism for $\tau$-topology (7.8.2). If the morphism of underlying rigid spaces of $f$ belongs to $\mathbf{A d}_{/ \mathfrak{T}^{\text {rig }}}\left(\operatorname{resp} . \mathbf{R f}_{/ \mathfrak{T}^{\text {rig }}}\right), f_{\tau}$ is the localisation morphism at $\mathfrak{T}^{\prime}(7.8)$ and then $\gamma_{f}$ is an isomorphism. If $g: \mathfrak{T}^{\prime \prime \text { rig }} \rightarrow \mathfrak{T}^{\prime \text { rig }}$ is another morphism of $\operatorname{RConv}(X / \mathfrak{S})$, one verifies that $\gamma_{g \circ f}=\gamma_{f} \circ f_{\tau}^{*}\left(\gamma_{g}\right)$.

By repeating the proof of 4.6 , we have the following description for a sheaf of $(X / \mathfrak{S})_{\mathrm{rconv}, \tau}$.

Proposition 8.9. For $\tau \in\{\mathrm{ad}, \mathrm{fppf}\}$, a sheaf $\mathscr{F}$ of $(X / \mathfrak{S})_{\mathrm{rconv}, \tau}$ is equivalent to the following data:

(i) For every object $\mathfrak{T}^{\text {rig }}$ of $\operatorname{RConv}(X / \mathfrak{S})$, a sheaf $\mathscr{F}_{\mathfrak{T}^{\text {rig }}}$ of $\mathfrak{T}_{\tau}^{\text {rig }}$,

(ii) For every morphism $f: \mathfrak{T}^{\prime \text { rig }} \rightarrow \mathfrak{T}^{\text {rig }}$ of $\operatorname{RConv}(X / \mathfrak{S})$, a morphism $\gamma_{f}$ (8.8.4),

subject to the following conditions

(a) If $f$ is the identity morphism of $(\mathfrak{T}, u), \gamma_{f}$ is the identity morphism.

(b) If the underlying morphism $f: \mathfrak{T}^{\prime \text { rig }} \rightarrow \mathfrak{T}^{\text {rig }}$ of coherent rigid spaces is a morphism of $\mathbf{A d}_{/ \mathfrak{T}^{\text {rig }}}$

(resp. $\left.\mathbf{R f}_{/ T^{\text {rig }}}\right)$, then $\gamma_{f}$ is an isomorphism.

(c) If $f$ and $g$ are two composable morphisms, then we have $\gamma_{g \circ f}=\gamma_{f} \circ f_{\tau}^{*}\left(\gamma_{g}\right)$.

8.10. Note that the fppf topology on $\operatorname{RConv}(X / \mathfrak{S})$ is finer than the admissible topology. Equipped with the fppf topology on the source and the admissible topology on the target, the identical functor id : $\operatorname{RConv}(X / \mathfrak{S}) \rightarrow \operatorname{RConv}(X / \mathfrak{S})$ is cocontinuous. By 2.12, it induces a morphism of topoi

$$
\alpha_{r}:(X / \mathfrak{S})_{\mathrm{rconv}, \mathrm{fppf}} \rightarrow(X / \mathfrak{S})_{\mathrm{rconv}, \mathrm{ad}} .
$$

If $\mathscr{F}$ is a sheaf of $(X / \mathfrak{S})_{\text {rconv,fppf }}, \alpha_{r *}(\mathscr{F})$ is equal to $\mathscr{F}$ as presheaves. If $\mathscr{G}$ is a sheaf of $(X / \mathfrak{S})_{\text {rconv,ad }}$, then $\alpha_{r}^{*}(\mathscr{G})$ is the sheafification of $\mathscr{G}$ with respect to the fppf topology. 
8.11. Equipped with the Zariski topology on the source and the admissible on the target, the canonical functor $\mathrm{Q}_{X / \mathfrak{S}}(8.3 .2)$ is clearly continuous. Since the functor $\mathrm{Q}_{X / \mathfrak{S} \text { ! }}$ and the shefification functor are exact (8.6), then we have a morphism of topoi

$$
\rho_{X / \mathfrak{S}}:(X / \mathfrak{S})_{\mathrm{rconv}, \mathrm{ad}} \rightarrow(X / \mathfrak{S})_{\text {conv,zar }}
$$

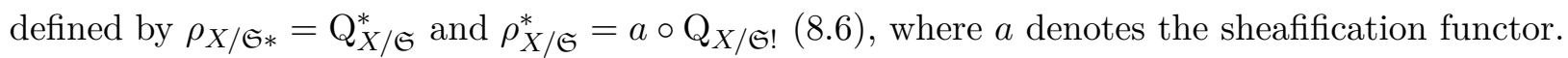

For any object $\mathfrak{T}$ of $\operatorname{Conv}(X / \mathfrak{S})$ and any sheaf $\mathscr{F}$ of $(X / \mathfrak{S})_{\text {rconv,ad }}$, we have (7.4)

$$
\left(\rho_{X / \mathfrak{S} *}(\mathscr{F})\right)_{\mathfrak{T}}=\rho_{\mathfrak{T} *}\left(\mathscr{F}_{\mathfrak{T}^{\mathrm{Tig}}}\right) .
$$

Let $f: \mathfrak{Z} \rightarrow \mathfrak{T}$ be a morphism of $\operatorname{Conv}(X / \mathfrak{S})$ and $\beta_{f^{\text {rig }}}, \gamma_{f^{\text {rig }}}\left(\right.$ resp. $\beta_{f}, \gamma_{f}$ ) transition morphisms of $\mathscr{F}$ associated to $f^{\text {rig }}$ (resp. $\rho_{X / \mathfrak{S} *}(\mathscr{F})$ associated to $f$ ) (4.5, 8.8). Via (8.11.2), we have $\beta_{f}=$ $\rho_{\mathfrak{T} *}\left(\beta_{f^{\text {rig }}}\right)$. Then we deduce that $\gamma_{f}$ coincides with the composition

$$
f_{\text {zar }}^{*}\left(\rho_{\mathfrak{T}^{*}}\left(\mathscr{F}_{\mathfrak{T}^{\text {rig }}}\right)\right) \rightarrow \rho_{\mathfrak{Z} *}\left(f_{\text {ad }}^{\text {rig } *}\left(\mathscr{F}_{\mathfrak{T}^{\text {rig }}}\right)\right) \stackrel{\rho_{\mathfrak{Z} *}\left(\gamma_{f^{\text {rig }}}\right)}{\longrightarrow} \rho_{\mathfrak{Z} *}\left(\mathscr{F}_{\mathfrak{Z}^{\text {rig }}}\right) .
$$

Proposition 8.12. Let $\mathscr{F}$ be a sheaf of $(X / \mathfrak{S})_{\mathrm{conv}, \mathrm{zar}}$ and $(\mathfrak{T}, u)$ an object of $\operatorname{Conv}(X / \mathfrak{S})$. There exists a canonical isomorphism



Proof. Let $\mathcal{U}$ be an object of $\mathbf{A d} / \mathfrak{T}^{\text {rig }}$ that we consider as an object of $\operatorname{RConv}(X / \mathfrak{S})$ via $r_{\mathfrak{T}^{\text {rig }}}(8.8 .1)$. By 8.4.1, $\left(\rho_{X / \mathfrak{S}}^{*}(\mathscr{F})\right)_{\mathfrak{T}^{\mathrm{rig}}}$ is the sheaf associated to the presheaf on $\mathbf{A d} / \mathfrak{T}^{\mathrm{rig}}$

$$
\mathcal{U} \mapsto \underset{(\mathfrak{Z}, g) \in\left(I_{\mathrm{Q}}^{\mathcal{U}}\right)^{\circ}}{\lim _{\longrightarrow}} \mathscr{F}(\mathfrak{Z}) .
$$

We denote by $J_{\mathrm{Q}}^{\mathcal{U}}$ the category of quadruple $\left(\mathfrak{T}^{\prime}, \varphi, \mathfrak{U}, g\right)$ consisting of an admissible blow-up $\left(\mathfrak{T}^{\prime}, \varphi\right)$ of $\mathfrak{T}$, an open formal subscheme $\mathfrak{U}$ of $\mathfrak{T}^{\prime}$ and an open immersion $g: \mathcal{U} \rightarrow \mathfrak{U}^{\text {rig }}$ over $\mathfrak{T}^{\text {rig }}$. A morphism $\left(\mathfrak{T}_{1}^{\prime}, \varphi_{1}, \mathfrak{U}_{1}, g_{1}\right)$ to $\left(\mathfrak{T}_{2}^{\prime}, \varphi_{2}, \mathfrak{U}_{2}, g_{2}\right)$ is a morphism $\mathfrak{T}_{1}^{\prime} \rightarrow \mathfrak{T}_{2}^{\prime}$ of $\mathbf{B}_{\mathfrak{T}}$ sending $\mathfrak{U}_{1}$ to $\mathfrak{U}_{2}$ compatible with $g_{1}, g_{2}$. The category $J_{\mathrm{Q}}^{\mathcal{U}}$ is clearly fibered over $\mathbf{B}_{\mathfrak{T}}$ :

$$
J_{\mathrm{Q}}^{\mathcal{U}} \rightarrow \mathbf{B}_{\mathfrak{T}}, \quad\left(\mathfrak{T}^{\prime}, \varphi, \mathfrak{U}, g\right) \mapsto\left(\mathfrak{T}^{\prime}, \varphi\right) .
$$

For any admissible blow-up $\left(\mathfrak{T}^{\prime}, \varphi\right)$ of $\mathfrak{T}$, we denotes its fiber by $J_{\mathrm{Q}, \mathfrak{T}^{\prime}}^{\mathcal{U}}$. The sheaf $\mu_{\varphi}^{*}\left(\mathscr{F}_{\mathfrak{T}^{\prime}}\right)$ is associated to the presheaf on $\mathbf{A d}_{/ \mathfrak{T}^{\mathrm{rig}}}$

$$
\mathcal{U} \mapsto \underset{(\mathfrak{U}, g) \in\left(\lim _{\mathrm{Q}, \mathfrak{T}^{\prime}}\right)^{\circ}}{\longrightarrow F}\left(r_{\mathfrak{T}}(\mathfrak{U})\right) .
$$

Then, the left hand side of $(8.12 .1)$ is the sheaf on $\mathbf{A d}_{/ \mathfrak{T}^{\text {rig }}}$ associated to the presheaf

$$
\mathcal{U} \mapsto \underset{\left(\mathfrak{T}^{\prime}, \varphi, \mathfrak{U}, g\right) \in\left(J_{\mathrm{Q}}^{\mathcal{U}}\right)^{\circ}}{\lim _{\longrightarrow}} \mathscr{F}\left(r_{\mathfrak{T}}(\mathfrak{U})\right) .
$$

We have a canonical functor (8.8)

$$
r: J_{\mathrm{Q}}^{\mathcal{U}} \rightarrow I_{\mathrm{Q}}^{\mathcal{U}} \quad\left(\mathfrak{T}^{\prime}, \varphi, \mathfrak{U}, g\right) \mapsto\left(r_{\mathfrak{T}}(\mathfrak{U}), r_{\mathfrak{T}^{\text {rig }}}(g)\right)
$$

We denote by $J$ the full subcategory of $J_{\mathrm{Q}}^{\mathcal{U}}$ consisting of objects such that $g$ is an isomorphism. Then each morphism of $J$ is Cartesian. Each category $\left(J_{\mathrm{Q}, \mathfrak{T}^{\prime}}^{\mathcal{U}}\right)^{\circ}$ is filtered by ([3] I 5.2). We deduce that $\left(J_{\mathrm{Q}}^{\mathcal{U}}\right)^{\circ}$ is filtered. It is clear that $J^{\circ}$ is cofinal in $\left(J_{\mathrm{Q}}^{\mathcal{U}}\right)^{\circ}$ and hence is filtered ([3] I 8.1 .3 a). 
To prove the assertion, it suffices to show that the induced functor $r: J^{\circ} \rightarrow\left(I_{\mathrm{Q}}^{\mathcal{U}}\right)^{\circ}$ is cofinal in the sense of ([3] I 8.1.1). By ([1] 4.2.2), for any object $\left(\mathfrak{Z}, g: \mathcal{U} \rightarrow \mathfrak{Z}^{\text {rig }}\right)$ of $I_{\mathrm{Q}}^{\mathcal{U}}$, there exists a morphism $h: \mathfrak{U} \rightarrow \mathfrak{Z}$ of $\operatorname{Conv}(X / \mathfrak{S})$ with an open formal subscheme $\mathfrak{U}$ of some admissible blow-up $\mathfrak{T}^{\prime}$ of $\mathfrak{T}$, such that $g=h^{\text {rig }}$, i.e. condition (F1) of ([3] I 8.1.3) is satisfied. Given an object $(\mathfrak{Z}, g)$ of $I_{\mathrm{Q}}^{\mathcal{U}}$, an object $\left(\mathfrak{T}^{\prime}, \varphi, \mathfrak{U}, h\right)$ of $J$ and two morphisms $f_{1}, f_{2}:\left(r_{\mathfrak{T}}(\mathfrak{U}), r_{\mathfrak{T}^{\text {rig }}}(h)\right) \rightarrow(\mathfrak{Z}, g)$, then $f_{1}^{\text {rig }}=f_{2}^{\text {rig }}$ in $\operatorname{RConv}(X / \mathfrak{S})$ since $h$ is an isomorphism. By ([1] 3.5.9), we deduce that $f_{1}=f_{2}$, i.e. condition (F2) of ([3] I 8.1.3) is satisfied. Then, the assertion follows from ([3] I 8.1.3 b).

Corollary 8.13. (i) The canonical morphism $\rho_{X / \mathfrak{S}}^{*} \rho_{X / \mathfrak{S} *} \rightarrow$ id is an isomorphism.

(ii) The functor $\rho_{X / \mathfrak{S} *}$ is fully faithful and its essential image consists of sheaves $\mathscr{F}=\left\{\mathscr{F}_{\mathfrak{T}}, \beta_{f}\right\}$ such that $\beta_{f}$ is an isomorphism for all morphism $f$ of $\mathbf{B}_{X / \mathfrak{S}}$.

Proof. (i) Let $\mathscr{F}$ be a sheaf of $(X / \mathfrak{S})_{\text {rconv,ad. Via } 8.11 .2)}$ and (8.12.1), we consider the evaluation of $\rho_{X / \mathfrak{S}}^{*} \rho_{X / \mathfrak{S} *}(\mathscr{F}) \rightarrow \mathscr{F}$ at an object $\mathfrak{T}^{\text {rig }}$ of $\operatorname{RConv}(X / \mathfrak{S})$



In view of the proof of 8.12 , the morphism $\mu_{\varphi}^{*}\left(\mu_{\varphi *}\left(\mathscr{F}_{\mathfrak{T}^{\text {rig }}}\right)\right) \rightarrow \mathscr{F}_{\mathfrak{T}^{\text {rig }}}$ deduced from $(8.13 .1)$ is nothing but the adjunction morphism. Then the assertion follows from ([1] 4.5.27 and 4.5.28).

(ii) By (i), the functor $\rho_{X / \mathfrak{S} *}$ is fully faithful. By (8.11), the essential image of $\rho_{X / \mathfrak{S} *}$ has the desired property. Let $\mathscr{G}$ be a sheaf of $(X / \mathfrak{S})_{\text {conv,zar }}$ satisfying the desired property. By ([1] 4.5.22 and 4.5.27), we deduce that the evaluation of the canonical morphism

$$
\mathscr{G} \rightarrow \rho_{X / \mathfrak{S} *} \rho_{X / \mathfrak{S}}^{*}(\mathscr{G})
$$

at each object of $\operatorname{RConv}(X / \mathfrak{S})$, is an isomorphism. Then the assertion follows.

8.14. Let $g: \mathfrak{S}^{\prime} \rightarrow \mathfrak{S}$ be a morphism of $\mathbf{S}^{\diamond}, X^{\prime}$ an $S^{\prime}$-scheme and $f: X^{\prime} \rightarrow X$ a morphism compatible with $g$ as in 4.8. The canonical functor $\varphi: \operatorname{Conv}\left(X^{\prime} / \mathfrak{S}^{\prime}\right) \rightarrow \operatorname{Conv}(X / \mathfrak{S})$ defined by $(\mathfrak{T}, u) \mapsto(\mathfrak{T}, f \circ u)$ (4.8.2), sends admissible blow-ups to admissible blow-ups. Then, $\varphi$ induces a functor that we denote by

$$
\psi: \operatorname{RConv}\left(X^{\prime} / \mathfrak{S}^{\prime}\right) \rightarrow \operatorname{RConv}(X / \mathfrak{S}) .
$$

Since $\varphi$ commutes with fiber products, the same holds for $\psi$ by 8.6. In view of 4.9)(i) and (8.3.1), one verifies that the functor $\psi$ is continuous and cocontinuous for admissible (resp. fppf) topology in the same way as in 4.9 ,

By 2.12, the functor $\psi$ (8.14.1) induces morphisms of topoi

$$
f_{\text {rconv }, \tau}:\left(X^{\prime} / \mathfrak{S}^{\prime}\right)_{\mathrm{rconv}, \tau} \rightarrow(X / \mathfrak{S})_{\mathrm{rconv}, \tau}, \quad \tau \in\{\operatorname{ad}, \mathrm{fppf}\}
$$

such that the pullback functor is induced by the composition with $\psi$. For a sheaf $\mathscr{F}$ of $(X / \mathfrak{S})_{\text {rconv }, \tau}$ and an object $\mathfrak{T}$ of $\operatorname{RConv}\left(X^{\prime} / \mathfrak{S}^{\prime}\right)$, we have

$$
\left(f_{\text {rconv }, \tau}^{*}(\mathscr{F})\right)_{\mathfrak{T}_{\text {rig }}}=\mathscr{F}_{\psi\left(\mathfrak{T}^{\text {rig }}\right)} .
$$

For any morphism $g$ of $\operatorname{RConv}\left(X^{\prime} / \mathfrak{S}^{\prime}\right)$, the transition morphism of $f_{\text {rconv }, \tau}^{*}(\mathscr{F})$ associated to $g$ is equal to the transition morphism of $\mathscr{F}$ associated to $\psi(g)$.

In view of the description of inverse image functors, we deduce the following result. 
Corollary 8.15. Keep the assumption and notation of 4.8 and of [8.14. The diagram

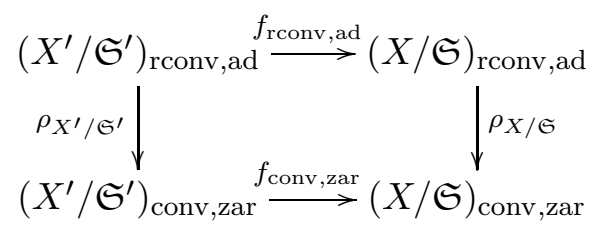

is commutative up to canonical isomorphisms.

8.16. We set $\mathscr{O}_{X / \mathfrak{S}}^{\mathrm{rig}}=\rho_{X / \mathfrak{S}}^{*}\left(\mathscr{O}_{X / \mathfrak{S}}\left[\frac{1}{p}\right]\right)$. By $8.12(\mathrm{i})$, for any object $\mathfrak{T}^{\text {rig }}$ of $\operatorname{RConv}(X / \mathfrak{S})$, we have a canonical isomorphism

$$
\left(\mathscr{O}_{X / \mathfrak{S}}^{\text {rig }}\right)_{\mathfrak{T}^{\text {rig }}} \stackrel{\sim}{\rightarrow} \mathscr{O}_{\mathfrak{T}^{\text {rig }}} .
$$

Then by fppf descent ([1] 5.11.11), the presheaf $\mathscr{O}_{X / \mathfrak{S}}^{\mathrm{rig}}$ is also a sheaf for the fppf (resp. admissible)

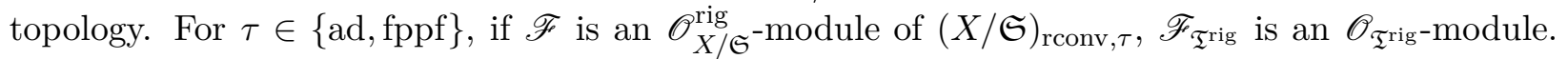
For any morphism $f: \mathfrak{T}^{\prime \text { rig }} \rightarrow \mathfrak{T}^{\text {rig }}$ of $\operatorname{RConv}(X / \mathfrak{S})$, the transition morphism $\gamma_{f}$ (8.9) extends to an $\mathscr{O}_{\mathfrak{T}^{\prime} \text { rig-linear morphism (7.8) }}$

$$
c_{f}: f_{\tau}^{*}\left(\mathscr{F}_{\mathfrak{T}^{\text {rig }}}\right) \rightarrow \mathscr{F}_{\mathfrak{T}^{\prime \text { rig }}} .
$$

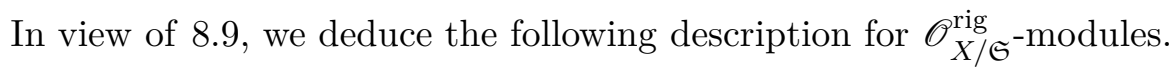

Proposition 8.17. For $\tau \in\{$ zar,fppf $\}$, an $\mathscr{O}_{X / \mathfrak{S}}^{\mathrm{rig}}$-module of $(X / \mathfrak{S})_{\mathrm{rconv}, \tau}$ is equivalent to the following data:

(i) For every object $\mathfrak{T}^{\text {rig }}$ of $\operatorname{RConv}(X / \mathfrak{S})$, an $\mathscr{O}_{\mathfrak{T}^{\text {rig }}}$-module $\mathscr{F}_{\mathfrak{T}}$ of $\mathfrak{T}_{\tau}$,

(ii) For every morphism $f: \mathfrak{T}^{\prime \text { rig }} \rightarrow \mathfrak{T}^{\text {rig }}$ of $\operatorname{RConv}(X / \mathfrak{S})$, an $\mathscr{O}_{\mathfrak{T}^{\prime} \text { rig }}$-linear morphism $c_{f}$ (8.16.2). which is subject to the following conditions

(a) If $f$ is the identity morphism, then $c_{f}$ is the identity.

(b) If the underlying morphism $f: \mathfrak{T}^{\prime \text { rig }} \rightarrow \mathfrak{T}^{\text {rig }}$ of coherent rigid spaces is a morphism of $\mathbf{A d}_{/ \mathfrak{T}^{\text {rig }}}$ (resp. $\left.\mathbf{R f}_{/ T^{\text {rig }}}\right)$, then $c_{f}$ is an isomorphism.

(c) If $f$ and $g$ are two composable morphisms, then we have $c_{g \circ f}=c_{f} \circ f_{\tau}^{*}\left(c_{g}\right)$.

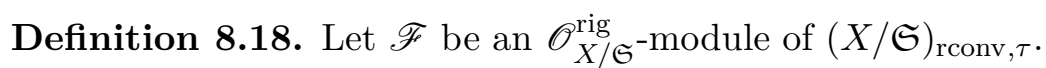

(i) We say that $\mathscr{F}$ is coherent if for every object $\mathfrak{T}^{\text {rig }}$ of $\operatorname{RConv}(X / \mathfrak{S}), \mathscr{F}_{\mathfrak{T}^{\text {rig }}}$ is coherent (7.9).

(ii) We say that $\mathscr{F}$ is a crystal if for every morphism $f$ of $\operatorname{RConv}(X / \mathfrak{S}), c_{f}$ is an isomorphism.

By fppf descent ([1] 5.11.11), the direct image and inverse image functors of $\alpha_{r}$ induce equaivalences of categories quais-inverse to each others between the category of coherent crystals of $\mathscr{O}_{X / \mathfrak{S}^{-}}^{\text {rig }}$ modules of $(X / \mathfrak{S})_{\text {rconv,ad }}$ and of $(X / \mathfrak{S})_{\text {rconv,fppf }}$.

Proposition 8.19. The direct image and inverse image functors of $\rho_{X / \mathfrak{S}}$ induce equivalences of categories quasi-inverse to each other between the category of coherent crystals of $\mathscr{O}_{X / \mathfrak{S}}\left[\frac{1}{p}\right]$-modules of $(X / \mathfrak{S})_{\text {conv,zar }}$ and that of coherent crystals of $\mathscr{O}_{X / \mathfrak{S}}^{\mathrm{rig}}$-modules of $(X / \mathfrak{S})_{\mathrm{rconv}, \mathrm{ad}}$.

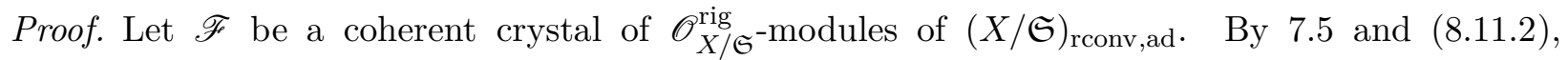
$\rho_{X / \mathfrak{S} *}(\mathscr{F})$ is coherent. In view of (7.6.2) and (8.11.3), we deduce that it is also a crystal. By $8.13(\mathrm{i}), \rho_{X / \mathfrak{S}}^{*} \rho_{X / \mathfrak{S} *}(\mathscr{F}) \rightarrow \mathscr{F}$ is an isomorphism. 
Let $\mathscr{G}$ be a coherent crystal of $\mathscr{O}_{X / \mathfrak{S}}\left[\frac{1}{p}\right]$-modules of $(X / \mathfrak{S})_{\text {conv,zar }}$ and $\mathscr{H}=\rho_{X / \mathfrak{S}}^{*}(\mathscr{G})$. By Tate's acyclicity ([1] 3.5.5), $\mathscr{G}$ is contained in the essential image of $\rho_{X / \mathfrak{S} *}$ (8.13)(ii)). Then we have a canonical isomorphism $\mathscr{G} \stackrel{\sim}{\rightarrow} \rho_{X / \mathfrak{S} *}(\mathscr{H})$ (8.13.2). By (8.12), (7.6.2) and (8.11.3), we deduce that $\mathscr{H}$ is coherent and is a crystal. Then the assertion follows.

8.20. Let $g: X \rightarrow Y$ be a morphism of $S$-schemes, $\mathfrak{T}$ be an object of $\operatorname{Conv}(Y / \mathfrak{S})$ and $\mathfrak{T}^{\text {rig }}$ its image in $\operatorname{RConv}(Y / \mathfrak{S})$. By fppf descent for morphisms of coherent rigid spaces ([1] 5.12.1), the presheaf associated to $\mathfrak{T}^{\text {rig }}$ is a sheaf for the fppf (resp. Zariski) topology that we denote by $\widetilde{\mathfrak{T}}^{\text {rig }}$. We set $X_{T_{0}}=X \times_{Y} T_{0}$ and for $\tau \in\{\operatorname{ad}$, fppf $\}$, we denote by

$$
\begin{array}{cl}
g_{X / \mathfrak{T}, \tau}: & \left(X_{T_{0}} / \mathfrak{T}\right)_{\mathrm{rconv}, \tau} \rightarrow\left(T_{0} / \mathfrak{T}\right)_{\mathrm{rconv}, \tau}, \\
\omega_{\mathfrak{T} \text { rig }}: & \left(X_{T_{0}} / \mathfrak{T}\right)_{\mathrm{rconv}, \tau} \rightarrow(X / \mathfrak{S})_{\mathrm{rconv}, \tau}
\end{array}
$$

the functorial morphisms of topoi (8.14.2).

By repeating arguments of $\S 5$, we prove the following results in the rigid convergent topos.

Lemma 8.21 (5.2). Keep the notation of 8.20. There exists a canonical equivalence of topoi:

$$
(X / \mathfrak{S})_{\text {rconv }, \tau / g_{\text {rconv }, \tau}^{*}\left(\widetilde{\mathfrak{T}}^{\mathrm{rig}}\right)} \stackrel{\sim}{\rightarrow}\left(X_{T_{0}} / \mathfrak{T}\right)_{\mathrm{rconv}, \tau}
$$

which identifies the localisation morphism and $\omega_{\mathfrak{T} \text { rig }}$.

Lemma 8.22 (5.3). For any $\mathscr{O}_{X / \mathfrak{S}}^{\mathrm{rig}}$-module $E$ of $(X / \mathfrak{S})_{\mathrm{rconv}, \tau}$, there exists a canonical isomorphism in $\mathrm{D}^{+}\left(\mathfrak{T}_{\tau}^{\text {rig }}, \mathscr{O}_{\mathfrak{T}^{\text {rig }}}\right)$

$$
\left(\mathrm{R} g_{\text {rconv }, \tau *}(E)\right)_{\mathfrak{T}^{\text {rig }}} \stackrel{\sim}{\rightarrow}\left(\mathrm{R} g_{X / \mathfrak{T}, \tau *}\left(\omega_{\mathfrak{T}^{\text {rig }}}^{*}(E)\right)\right)_{\mathfrak{T}^{\text {rig }}} .
$$

Corollary 8.23 (5.6). Let $\mathfrak{S}^{\prime} \rightarrow \mathfrak{S}$ be a morphism of $\mathbf{S}^{\diamond}, Y^{\prime}$ an $S^{\prime}$-scheme and $h: Y^{\prime} \rightarrow Y a$ morphism compatible with $S^{\prime} \rightarrow S$. We set $X^{\prime}=X \times_{Y} Y^{\prime}$ and we denote by $g^{\prime}: X^{\prime} \rightarrow Y^{\prime}$ and $h^{\prime}: X^{\prime} \rightarrow X$ the canonical morphisms:

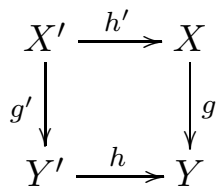

Then, for any $\mathscr{O}_{X / \mathfrak{S}}\left[\frac{1}{p}\right]$-module $E$ of $(X / \mathfrak{S})_{\mathrm{conv}, \tau}$, the base change morphism

$$
h_{\mathrm{rconv}, \tau}^{*}\left(\mathrm{R} g_{\mathrm{rconv}, \tau *}(E)\right) \stackrel{\sim}{\rightarrow} \mathrm{R} g_{\mathrm{rconv}, \tau *}^{\prime}\left(h_{\mathrm{rconv}, \tau}^{\prime *}(E)\right),
$$

is an isomorphism.

Corollary 8.24 (5.5). Let $\mathscr{E}$ be a coherent crystal of $\mathscr{O}_{X / \mathfrak{S}}^{\mathrm{rig}}$-modules of $(X / \mathfrak{S})_{\mathrm{rconv}, \mathrm{fppf}}$. Then we have 8.10.1)

$$
\mathrm{R}^{i} \alpha_{r *}(\mathscr{E})=0 \quad \forall i \geq 1 .
$$

Corollary 8.25. Let $\mathscr{E}$ be a coherent crystal of $\mathscr{O}_{X / \mathfrak{S}}^{\mathrm{rig}}$-modules of $(X / \mathfrak{S})_{\mathrm{rconv}, \mathrm{ad}}$. Then we have

$$
\mathrm{R}^{i} \rho_{X / \mathfrak{S} *}(\mathscr{E})=0 \quad \forall i \geq 1 .
$$


Proof. By [8.21, the Zariski sheaf $\mathrm{R}^{i} \rho_{X / \mathfrak{S} *}(\mathscr{E})$ on $\operatorname{RConv}(X / \mathfrak{S})$ is associated to the presheaf

$$
\mathfrak{T}^{\text {rig }} \mapsto \mathrm{H}^{i}\left(\left(T_{0} / \mathfrak{T}\right)_{\text {rconv, ad }},\left.\mathscr{E}\right|_{\widetilde{T}_{\text {rig }}}\right) .
$$

By ([3] V 4.3 and III 4.1), we can replace $\operatorname{RConv}(X / \mathfrak{S})$ by the full subcategory of objects whose underlying rigid space is affinoid, and it suffices to show that for such an object $\mathfrak{T}^{\text {rig }}$

$$
\mathrm{H}^{i}\left(\left(T_{0} / \mathfrak{T}\right)_{\text {conv,ad }},\left.\mathscr{E}\right|_{\mathfrak{T}}\right)
$$

vanishes for $i \geq 1$. Let $\mathscr{U}=\left\{\mathfrak{Z}_{i}^{\text {rig }} \rightarrow \mathfrak{Z}^{\text {rig }}\right\}_{i=1}^{m}$ be an admissible covering by affinoids of an affinoids $\mathfrak{Z}^{\text {rig }}$ in $\mathbf{R}_{/ \mathfrak{T}^{\text {rig }}}$. The Čech cohomology $\check{\mathrm{H}}^{i}\left(\mathscr{U},\left.\mathscr{E}\right|_{\mathfrak{Z}^{\text {rig }}}\right)$ is isomorphic to the cohomology $\mathrm{H}^{i}\left(\mathfrak{Z}_{\text {ad }}^{\text {rig }}, \mathscr{E}_{\mathfrak{Z}^{\text {rig }}}\right)$ which vanishes by ([1 4.8.26). Since each admissible covering of $\mathfrak{Z}^{\text {rig }}$ admits a refinement by finitely many affinoids, the vanishing of (8.25.2) follows from ([35] 21.11.9).

\section{Higher DireCt images of a CONVERGEnt isocrystal}

9.1. Let $X$ be a $k$-scheme locally of finite type. For $\tau \in\{\mathrm{ad}$,fppf $\}$, the Frobenius homomorphism $\sigma: \mathrm{W} \rightarrow \mathrm{W}$ induces a morphism of topoi $\left(X^{\prime} / \mathrm{W}\right)_{\mathrm{rconv}, \tau} \rightarrow(X / \mathrm{W})_{\mathrm{rconv}, \tau}$ (8.14.2). For any sheaf $\mathscr{E}$ of $(X / \mathrm{W})_{\text {rconv }, \tau}$, we denote by $\mathscr{E}^{\prime}$ the inverse image of $\mathscr{E}$ to $\left(X^{\prime} / \mathrm{W}\right)_{\text {rconv }, \tau}$.

As in 6.14, we call convergent $F$-isocrystal of $(X / \mathrm{W})_{\mathrm{rconv}, \tau}$ a pair $(\mathscr{E}, \varphi)$ consisting of a coherent

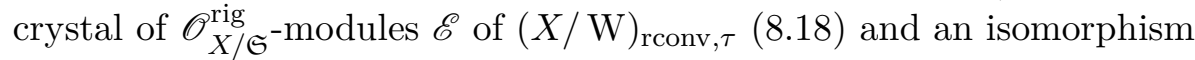

$$
\varphi: F_{X / k, \text { rconv }, \tau}^{*}\left(\mathscr{E}^{\prime}\right) \stackrel{\sim}{\rightarrow} \mathscr{E} .
$$

In this section, we prove the following result about the higher direct image of a convergent $(F-)$ isocrystal of rigid convergent topos.

Theorem 9.2. Let $g: X \rightarrow Y$ be a smooth proper morphism of $k$-schemes locally of finite type and $\mathscr{E}$ (resp. $(\mathscr{E}, \varphi)$ ) a convergent isocrystal (resp. F-isocrystal) of $(X / \mathrm{W})_{\mathrm{rconv}, \tau}$. Then, $\mathrm{R}^{i} g_{\mathrm{rconv}, \tau *}(\mathscr{E})$ (resp. $\left.\left(\mathrm{R}^{i} g_{\mathrm{rconv}, \tau *}(\mathscr{E}), \mathrm{R}^{i} g_{\mathrm{rconv}, \tau *}(\varphi)\right)\right)$ is a convergent isocrystal (resp. F-isocrystal) of $(Y / \mathrm{W})_{\mathrm{rconv}, \tau}$.

By 8.25, 9.2 and arguments of 6.13, we deduce the variant for the convergent topos.

Corollary 9.3. Keep the assumption of 9.2. The higher direct image of a convergent isocrystal (resp. F-isocrystal) of $(X / \mathrm{W})_{\text {conv,zar }}(6.14)$ is a convergent isocrystal (resp. F-isocrystal) of $(Y / \mathrm{W})_{\text {conv,zar }}$.

Proposition 9.4. Keep the notation and assumption of 9.2, If $Y$ is moreover smooth over $k$, then $\mathrm{R}^{i} g_{\mathrm{rconv}, \tau *}(\mathscr{E})$ is a coherent crystal of $\mathscr{O}_{Y / \mathrm{W}}^{\mathrm{rig}}$-modules.

Proof. We first prove the assertion for the admissible topology. The sheaf $\mathscr{F}=\rho_{X / \mathrm{W} *}(\mathscr{E})$ is a coherent crystal of $\mathscr{O}_{X / \mathrm{W}}^{\mathrm{rig}}$-modules of $(X / \mathrm{W})_{\text {conv,zar }}$ and $\rho_{X / \mathrm{W}}^{*}(\mathscr{F}) \stackrel{\sim}{\rightarrow} \mathscr{E}(\underline{8.19})$. By $\left[6.10, \mathrm{R}^{i} g_{\text {conv,zar } *(\mathscr{F})}\right.$ is a coherent crystal of $\mathscr{O}_{Y / \mathrm{W}}^{\mathrm{rig}}$-modules of $(Y / \mathrm{W})_{\text {conv,zar }}$. We consider the canonical morphisms

$$
\mathrm{R}^{i} g_{\text {conv }, \mathrm{zar} *}(\mathscr{F}) \stackrel{\sim}{\rightarrow} \rho_{Y / \mathrm{W} *} \rho_{Y / \mathrm{W}}^{*}\left(\mathrm{R}^{i} g_{\text {conv }, \mathrm{zar} *}(\mathscr{F})\right) \rightarrow \rho_{Y / \mathrm{W} *}\left(\mathrm{R}^{i} g_{\mathrm{rconv}, \mathrm{ad} *}(\mathscr{E})\right)
$$

where the first arrow is an isomorphism by 8.19 and second arrow is induced by the base change

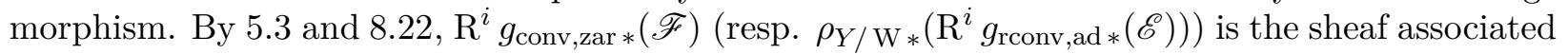
to the presheaf on $\operatorname{Conv}(Y / \mathrm{W})$

$$
\begin{aligned}
\mathfrak{T} & \mapsto \mathrm{H}^{i}\left(\left(X_{T_{0}} / \mathfrak{T}\right)_{\text {conv }, \mathrm{zar}}, \omega_{\mathfrak{T}}^{*}(\mathscr{F})\right), \\
\text { (resp. } & \left.\mathfrak{T} \mapsto \mathrm{H}^{i}\left(\left(X_{T_{0}} / \mathfrak{T}\right)_{\text {rconv }, \mathrm{ad}}, \omega_{\mathfrak{T} \text { rig }}^{*}(\mathscr{E})\right)\right) .
\end{aligned}
$$


By 8.25, the canonical morphism

$$
\mathrm{H}^{i}\left(\left(X_{T_{0}} / \mathfrak{T}\right)_{\text {conv }, \mathrm{zar}}, \omega_{\mathfrak{T}}^{*}(\mathscr{F})\right) \stackrel{\sim}{\rightarrow} \mathrm{H}^{i}\left(\left(X_{T_{0}} / \mathfrak{T}\right)_{\text {rconv,ad }}, \omega_{\mathfrak{T}^{\text {rig }}}^{*}(\mathscr{E})\right)
$$

is an isomorphism. The composition (9.4.1) is induced by above morphisms and hence is an isomorphism. In view of the definition of $\rho_{Y / \mathrm{W} *}(\underline{8.11})$, we deduce that $\rho_{Y / \mathrm{W}}^{*}\left(\mathrm{R}^{i} g_{\text {conv,zar* }}(\mathscr{F})\right) \stackrel{\sim}{\rightarrow}$ $\mathrm{R}^{i} g_{\mathrm{rconv}, \mathrm{ad} *}(\mathscr{E})$ by (9.4.1). Then the assertion for admissible topology follows from 8.19.

Using 8.24, one verifies the proposition for fppf topology by comparing $\mathrm{R}^{i} g_{\mathrm{rconv}, \text { ad }}(-)$ and $\mathrm{R}^{i} g_{\mathrm{rconv}, \mathrm{fppf}}(-)$ in a similar way as above.

9.5. To prove 9.2 , we use the proper descent for convergent isocrystals developed by Ogus in [26]. Let $\mathfrak{T}$ be a formal $\mathrm{W}$-scheme of finite type and $f: Z \rightarrow T_{0}$ a projective and surjective morphism. Then $f$ factors through a closed immersion $Z \rightarrow \mathbb{P}_{T_{0}}^{N}$ for some integer $N \geq 1$. We denote by $\mathbb{P}_{\mathfrak{T}}^{N}$ the formal W-scheme associated to the inductive system $\left(\mathbb{P}_{\mathfrak{T}_{n}}^{N}\right)_{n \geq 1}$. By 2.10, we can construct a family of adic formal $\mathbb{P}_{\mathfrak{T}}^{N}$-schemes $\left\{\mathfrak{T}_{Z, n}\left(\mathbb{P}_{\mathfrak{T}}^{N}\right)\right\}_{n \geq 0}$. Based on the following result, Ogus showed that a proper surjective $k$-morphism satisfies descent for convergent isocrystals ([26] 4.6).

Theorem 9.6 ([26] 4.7, 4.8). For $n$ large enough, the morphism $\mathfrak{T}_{Z, n}\left(\mathbb{P}_{\mathfrak{T}}^{N}\right) \rightarrow \mathfrak{T}$ is faithfully rig-flat (2.7).

A variant of 6.6 holds for rigid convergent topos:

Proposition 9.7. For every locally of finite type $k$-scheme $X$, the morphism

$$
F_{X / k, \text { rconv,fppf }}:(X / \mathrm{W})_{\text {rconv,fpp }} \rightarrow\left(X^{\prime} / \mathrm{W}\right)_{\text {rconv,fppf }}
$$

is an equivalence of topoi.

By 6.3 and (8.3.1), the canonical functor $\operatorname{RConv}(X / \mathrm{W}) \rightarrow \operatorname{RConv}\left(X^{\prime} / \mathrm{W}\right)$ induced by $F_{X / k}$ (8.14.1) is fully faithful. In view of [2.13, 6.4 and 8.11, the assertion follows.

9.8. Proof of 9.2. We prove the assertion for convergent isocrystals. Then the assertion for convergent $F$-isocrystals follows from 9.7 and a similar argument as in 6.15, The question being local (8.23), we may assume that $Y$ is separated and of finite type by 8.23. Moreover, we may assume that $Y$ is reduced.

By applying alteration to each irreducible component of $Y$ ([15] 4.1), there exists a smooth $k$-scheme $\widetilde{Y}$ and a proper surjective $k$-morphism $\widetilde{Y} \rightarrow Y$. By Chow's lemma ([23] 5.6.1), there exists a surjective $k$-morphism $Z \rightarrow \tilde{Y}$ such that the composition $f: Z \rightarrow \tilde{Y} \rightarrow Y$ is projective

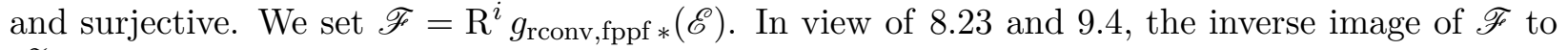
$(\tilde{Y} / \mathrm{W})_{\text {rconv,fppf }}$ is a coherent crystal. Then, so is $f_{\text {rconv,fppf }}^{*}(\mathscr{F})$.

Let $(\mathfrak{T}, u)$ be an object of $\operatorname{Conv}(Y / \mathrm{W})$. The morphism $f$ factor through a closed immersion $Z \rightarrow \mathbb{P}_{Y}^{N}$ for some integer $N \geq 1$. We set $T_{Z}=T_{0} \times_{Y} Z$. We take again the notation of 9.5 for the projective and surjective $k$-morphisms $T_{Z} \rightarrow T_{0}$. We choose an integer $n$ such that the morphism $\mathfrak{T}_{T_{Z}, n}\left(\mathbb{P}_{\mathfrak{T}}^{N}\right) \rightarrow \mathfrak{T}$ is faithfully rig-flat $(9.6)$. We set $\mathfrak{R}=\mathfrak{T}_{T_{Z}, n}\left(\mathbb{P}_{\mathfrak{T}}^{N}\right), \mathfrak{R}^{(1)}=\mathfrak{R} \times \mathfrak{T} \mathfrak{R}$ and denote by $p_{1}, p_{2}: \mathfrak{R}^{(1)} \rightarrow \mathfrak{R}$ two projections.

Note that $\mathfrak{R}$ and $\mathfrak{R}^{(1)}$ define objects of $\operatorname{Conv}(Z / \mathrm{W})$ by (2.10.2) and then of $\operatorname{Conv}(Y / \mathrm{W})$. Moreover, $\left\{\mathfrak{R}^{\text {rig }} \rightarrow \mathfrak{T}^{\text {rig }}\right\}$ defines a fppf covering of $\operatorname{RConv}(V / \mathrm{W})$. Since $f_{\text {rconv,fppf }}^{*}(\mathscr{F})$ is a coherent crystal of $\mathscr{O}_{Z / \mathrm{W}}^{\mathrm{rig}}$-modules, following modules are coherent

$$
\mathscr{F}_{\mathfrak{R}^{\text {rig }}}=\left(f_{\text {rconv }, \text { fppf }}^{*}(\mathscr{F})\right)_{\mathfrak{R}^{\text {rig }}}, \quad \mathscr{F}_{\mathfrak{R}^{(1), \text { rig }}}=\left(f_{\text {rconv }, \text { fppf }}^{*}(\mathscr{F})\right)_{\mathfrak{R}^{(1), \text { rig }}},
$$


and we have isomorphisms

$$
p_{2}^{\text {rig } *}\left(\mathscr{F}_{\mathfrak{R}^{\text {rig }}}\right) \stackrel{\sim}{\rightarrow} \mathscr{F}_{\mathfrak{R}^{(1), \text { rig }}} \stackrel{\sim}{\leftarrow} p_{1}^{\text {rig } *}\left(\mathscr{F}_{\mathfrak{R}^{\text {rig }}}\right) .
$$

Then we obtain a descent data on $\mathscr{F}_{\mathfrak{R}^{\text {rig }}}$ for the fppf covering $\left\{u: \mathfrak{R}^{\text {rig }} \rightarrow \mathfrak{T}^{\text {rig }}\right\}$. There exists a coherent $\mathscr{O}_{\mathfrak{T}^{\text {rig }}}$-module $\mathscr{M}$ and an isomorphism $u^{*}(\mathscr{M}) \stackrel{\sim}{\rightarrow} \mathscr{F}_{\mathfrak{R}^{\text {rig }}}$ by ([1] 5.11.11).

On the other hand, since $\mathscr{F}$ is a sheaf for fppf topology, we have an exact sequence

$$
0 \rightarrow \mathscr{F}\left(\mathfrak{T}^{\text {rig }}\right) \rightarrow \mathscr{F}\left(\mathfrak{R}^{\text {rig }}\right) \rightarrow \mathscr{F}\left(\mathfrak{R}^{(1), \text { rig }}\right) .
$$

Then we deduce that $\mathscr{F}_{\mathbb{T}_{\text {rig }}}$ is isomorphic to $\mathscr{M}$ and hence is coherent.

Let $g: \mathfrak{T}^{\prime} \rightarrow \mathfrak{T}$ be a morphism of $\operatorname{Conv}(Y / \mathrm{W})$. Choose an integer $n$ large enough such that $\mathfrak{R}^{\prime}=\mathfrak{T}_{T_{Z}^{\prime}, n}\left(\mathbb{P}_{\mathfrak{T}^{\prime}}^{N}\right) \rightarrow \mathfrak{T}^{\prime}$ and $\mathfrak{R}=\mathfrak{T}_{T_{Z}, n}\left(\mathbb{P}_{\mathfrak{T}}^{N}\right) \rightarrow \mathfrak{T}$ are faithfully rig-flat. Since the construction of $\mathfrak{R}$ is functorial, we have a W-morphism $h: \mathfrak{R}^{\prime} \rightarrow \mathfrak{R}$ compatible with $g$. Moreover, $h$ induces a morphism of $\operatorname{Conv}(Z / \mathrm{W})$. The transition morphism of $f_{\text {rconv,fppf }}^{*}(\mathscr{F})$ associated to $h^{\text {rig }}$ is an isomorphism. By fppf descent, we deduce that the transition morphism $c_{g^{\text {rig }}}$ of $\mathscr{F}$ associated to $g^{\text {rig }}$ is an isomorphism (cf. [34] 8.15). Then $\mathscr{F}$ is a crystal and the theorem follows.

\section{REFERENCES}

[1] A. Abbes, Éléments de Géométrie Rigide: Volume I. Construction et étude géométrique des espaces rigides. Springer-Verlag, (2010).

[2] A. Abbes, M. Gros, T. Tsuji, The p-adic Simpson correspondence, Annals of Mathematics Studies, Vol. 193 (2016).

[3] M. Artin, A. Grothendieck, J. L. Verdier, Théorie des topos et cohomologie étale des schémas, SGA 4, SpringerVerlag, Tome 1, LNM 269 (1972) ; Tome 2, LNM 270 (1972) ; Tome 3, LNM 305 (1973).

[4] P. Berthelot, Cohomologie cristalline des schémas de caractéristique $p>0$, Springer-Verlag, LNM 407, (1974).

[5] P. Berthelot, Géométrie rigide et cohomologie des variétés algébriques de caractéristique p, Mem. Soc. Math. France no. 23 (1986), 7-32.

[6] P. Berthelot, Cohomologie rigide et cohomologie ridige à supports propres, première partie, preprint (1996).

[7] P. Berthelot, D-modules arithmétiques. I. Opérateurs différentiels de niveau fini, Ann. Sci. École Norm. Sup. (4) 29 (1996), no. 2, 185-272.

[8] P. Berthelot, D-modules arithmétiques. II. Descente par Frobenius. Mém. Soc. Math. Fr. No. 81 (2000).

[9] P. Berthelot, L. Breen and W. Messing, Théorie de Dieudonné Cristalline II, LNM 930, Springer-Verlag, (1982).

[10] P. Berthelot, A. Grothendieck, L. Illusie, Théorie des intersections et théorème de Riemann-Roch, SGA 6, LNM 225, Springer-Verlag (1971).

[11] P. Berthelot, A. Ogus, Notes on Crystalline Cohomology (MN-21). Princeton University Press, (2015).

[12] S. Bosch, U. Görtz, Coherent modules and their descent on relative rigid spaces, J. reine angew. Math. 495 (1998), 119-134.

[13] N. Bourbaki, Algèbre commutative, Chapitres 1-9, Hermann (1985).

[14] D. Caro, Sur la préservation de la surconvergence par l'image directe d'un morphisme propre et lisse, Ann. Sci. Éc. Norm. Supér. (4) 48 (2015), no. 1, 131-169.

[15] A. J. de Jong. Smoothness, semi-stability and alterations, Publications Mathématiques de l'IHÉS 83 (1996): 51-93.

[16] M. Demazure, A. Grothendieck. Schémas en groupes, SGA3. LNM 151-153, Springer-Verlag, (1970).

[17] J.-Y. Étesse Images directes I: Espaces rigides analytiques et images directes, J. Théor. Nombres Bordeaux 24 (2012), no. 1, 101-151.

[18] P. Gabriel, M. Zisman, Calculus of fractions and homotopy theory, Ergebnisse der Mathematik, Bd. 35, SpringerVerlag (1967).

[19] L. Illusie, Frobenius et dégénérescence de Hodge, in Introduction à la théorie de Hodge, Panoramas et Synthèses, vol. 3, Soc. Math. de France, (1996), pp. 113-168.

[20] N. Katz, Nilpotent connections and the monodromy theorem: Applications of a result of Turrittin, Publ. Math. IHÉS 39 (1970): 175-232. 
[21] K. Kedlaya, Good formal structures for flat meromorphic connections, I: Surfaces, Duke Math. J. 154 (2010), no. 2, 343-418.

[22] A. Grothendieck, J. Dieudonné, Éléments de Géométrie Algébrique I, Seconde édition, Springer-Verlag (1971).

[23] A. Grothendieck, J.A. Dieudonné, Éléments de Géométrie Algébrique, II Étude globale élémentaire de quelques classes de morphismes, Pub. Math. IHÉS 8 (1961).

[24] C. Lazda, Incarnations of Berthelot's conjecture, J. Number Theory (2016) 166, 137-157.

[25] M. Morrow, A note on higher direct images in crystalline cohomology, appendix to A Variational Tate Conjecture in crystalline cohomology, to appear in Journal of the European Mathematical Society.

[26] A. Ogus, F-isocrystals and de Rham cohomology. II. Convergent isocrystals. Duke Math. J. 51 (1984), no. 4, $765-850$.

[27] A. Ogus, The convergent topos in characteristic p. The Grothendieck Festschrift Volume III (2007): 133-162.

[28] A. Ogus, V. Vologodsky, Nonabelian Hodge theory in characteristic p, Publ. Math. IHÉS 106.1 (2007), 1-138.

[29] H. Oyama, PD Higgs crystals and Higgs cohomology in characteristic p, J. Algebraic Geom. 26 (2017), 735-802.

[30] A. Shiho, Crystalline fundamental groups. II. Log convergent cohomology and rigid cohomology. J. Math. Sci. Univ. Tokyo 9 (2002), no. 1, 1-163.

[31] A. Shiho, Relative log convergent cohomology and relative rigid cohomology I https://arxiv.org/abs/0707.1742.

[32] A. Shiho, Relative log convergent cohomology and relative rigid cohomology II https://arxiv.org/abs/0707.1743.

[33] N. Tsuzuki, On base change theorem and coherence in rigid cohomology, Doc. Math. extra vol. (2003), 891-918.

[34] D. Xu. Lifting the Cartier transform of Ogus-Vologodsky modulo $p^{n}$, arxiv 1705.06241.

[35] The Stacks Project Authors. Stacks Project. http://stacks.math.columbia.edu, 2017.

Daxin Xu, Department of Mathematics, California Institute of Technology, Pasadena, CA 91125.

E-mail address: daxinxu@caltech.edu 